\title{
The Status of Glueballs
}

\author{
Wolfgang Ochs \\ Max-Planck-Institut für Physik, Werner-Heisenberg-Institut, \\ Föhringer Ring 6, D-80805 München, Germany \\ E-mail: ochs@mpp.mpg.de
}

\begin{abstract}
Calculations within QCD (lattice and sum rules) find the lightest glueball to be a scalar and with mass in the range of about 1000-1700 MeV. Several phenomenological investigations are discussed which aim at the identification of the scalar meson nonets of lowest mass and the super-numerous states if any. Results on the flavour structure of the light scalars $f_{0}(500), f_{0}(980)$ and $f_{0}(1500)$ are presented; the evidence for $f_{0}(1370)$ is scrutinized. A significant surplus of leading clusters of neutral charge in gluon jets is found at LEP in comparison with MCs, possibly a direct signal for glueball production; further studies with more energetic jets at LHC are suggested. As a powerful tool in the identification of the scalar nonets or other multiplets, along with signals from glueballs we propose the exploration of symmetry relations for decay rates of $C=+1$ heavy quark states like $\chi_{c}$ or $\chi_{b}$. Results from $\chi_{c}$ decays are discussed; they are not in support of a tetra-quark substructure of $f_{0}(980)$. A minimal scenario for scalar quarkonium-glueball spectroscopy is presented.
\end{abstract}

1 Introduction $\quad 2$

2 QCD expectations for glueballs $\quad 4$

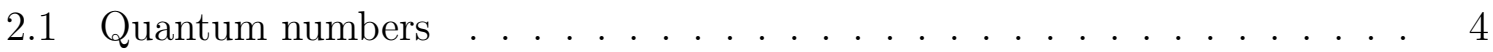

2.2 Lattice QCD. . . . . . . . . . . . . . . . . . . 5

2.3 QCD sum rules . . . . . . . . . . . . . . . . . 8

2.4 Conclusions from theory . . . . . . . . . . . . . . . 10

3 Experimental search strategies for glueballs $\quad 10$

3.1 Quark-gluon constituent structure of hadrons from their decays . . . . . 10

3.2 Enhanced and suppressed glueball production . . . . . . . . . . . . 15

3.3 Supernumerous states among $q \bar{q}$ nonets? . . . . . . . . . . . . . . . . 16

4 Spectroscopy of scalar mesons: $q \bar{q}$ nonets mixing with glueballs $\quad 17$

4.1 Route 1: $(q \bar{q})$ nonet - glueball mixing around $1500 \mathrm{MeV}$. . . . . . . . . . 17

4.2 Route 2: light glueball around $1 \mathrm{GeV}$. . . . . . . . . . . . . . . . 18

4.3 Route 3: two supernumerous states, broad glueball $f_{0}(1200-1600)$. . . 20 
5 Properties of $f_{0}(500) / \sigma$ - comparison with $K_{0}^{*}(800) / \kappa \quad 21$

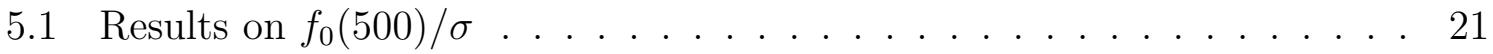

5.2 Comparing elastic $\pi \pi$ and $K \pi$ scattering - a problem with the $\kappa$ resonance 25

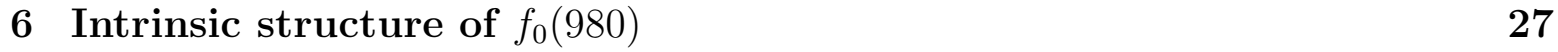

6.1 Mixing angle from various observables . . . . . . . . . . . . . . . . 27

6.2 Final result on mixing angle . . . . . . . . . . . . . . . . . . . . . . . . . 30

6.3 Possible gluonic contribution . . . . . . . . . . . . . . . . . 30

7 Experimental evidence for $f_{0}(1370)$ and $f_{0}(1500)$ - a reassessment $\quad 31$

7.1 Production in $p \bar{p}$ annihilation . . . . . . . . . . . . . . . . . . 31

7.2 Search in phase shift analysis of $\pi \pi$ scattering . . . . . . . . . . . . . 34

7.3 Decays of $D$ and $B$ into $f_{0}$ mesons . . . . . . . . . . . . . . . . 42

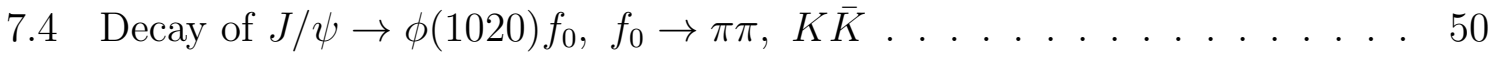

7.5 Central production in hadron hadron collisions . . . . . . . . . . . . . 50

7.6 Flavour structure of $f_{0}(1500)$ from its decays . . . . . . . . . . . . . . 54

7.7 Production in $\gamma \gamma$ scattering . . . . . . . . . . . . . . . . . . . 56

8 Gluon rich processes in comparison $\quad 57$

8.1 The Pomeron as an example for mixed gluon and quark components . . 58

8.2 Processes with identified gluons . . . . . . . . . . . . . . 59

9 Leading systems in gluon jets $\quad \mathbf{5 9}$

9.1 Rate of neutral clusters at LEP above Monte Carlo expectations . . . . . 60

9.2 Proposal for further measurements at the LHC . . . . . . . . . . . . . . . 62

$10[q \bar{q}]$ multiplets and glueballs from symmetry relations:

first results from $\chi_{c}$ decays $\quad 65$

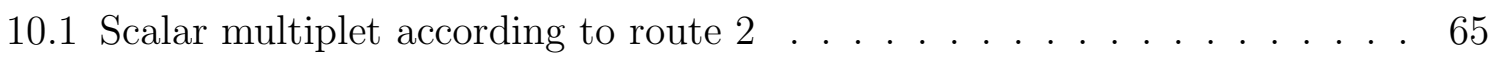

10.2 Scalar multiplet according to route 1 - tetraquark expectations . . . . . . 67

11 Summary and conclusions $\quad \mathbf{6 7}$

11.1 Is there any experimental evidence for the existence of glueballs? . . . . . 67

11.2 Are there supernumerous states in the scalar sector? . . . . . . . . . . 68

11.3 Are there hints towards a gluonic component in isoscalar mesons? . . . . 68

11.4 Future studies of symmetry relations in $\chi_{c}$ decays . . . . . . . . . . 68

11.5 A minimal scenario for scalar quarkonium-glueball spectroscopy . . . . . 69

$\begin{array}{ll}\text { List of reprinted figures } & 79\end{array}$

\section{Introduction}

The existence of "glueballs", bound states of gluons, is a consequence of the self-interaction of gluons within Quantumchromodynamics. Such states have been 
considered to appear in analogy to $q \bar{q}$ states in a quark-gluon theory of hadrons already in 1972 during the early development of QCD [1]. First specific scenarios for glueball spectroscopy on the basis of a quark-gluon field theory with hadrons as colour singlets have been developed by Fritzsch and Minkowski [2] assuming an analogy between massless gluons and photons. Much effort has been devoted during the last 40 years into the theoretical analysis as well as the experimental searches of this new type of hadrons.

While a consensus is reached about the existence and some properties of glueballs in a world without quarks, the details in the full theory are still controversial. Lattice QCD is a formulation of the quark-gluon gauge theory on a space-time lattice and it is suitable for describing hadronic phenomena in the non-perturbative regime with confined quarks. The theory starts directly from the QCD Lagrangian with no other parameters than quark masses and a mass scale. The lightest glueball has quantum numbers $J^{P C}=0^{++}$and a mass around $1700 \mathrm{MeV}$ in the theory without quarks but could become smaller in the full theory. Alternatively, information on the hadronic spectrum has been derived successfully from the "QCD sum rules". In this approach some phenomenological input parameters ("condensates") have to be determined from experiment but a wide spectrum of quantitative results in hadron physics has been established. The predictions from these two theoretical approaches on glueballs will be reported and summarized in section 2 .

Most of our attention concerning glueballs is focussed on the scalar sector with $J^{P C}=0^{++}$, as the lightest glueball is expected with these quantum numbers. The first aim of the experimental search is the identification of scalar nonets of $q \bar{q}$ bound states $(q=u, d, s)$ or other multiplets like those built from "tetraquarks", according to the classification within flavour $S U(3)_{f l}$ symmetry. Glueballs will then show up as super-numerous states. Unfortunately, it is just this scalar sector whose identification is still "tentative" while the other multiplets are quite well established [3]. Therefore, additional strategies, like the study of "gluon rich" processes have attracted attention. We present strategies following such investigations and describe different scenarios for a scalar quark-gluon spectroscopy in sections 3 and 4 .

It turns out that the validity of such scenarios depends crucially on the existence of certain scalar states and their specific properties. Therefore, in the subsequent sections 5 - 7 we analyze in greater detail the experimental data on production and decay of the isoscalar states which could have a gluonic contribution mixed in and analyze their flavour structure. The Particle Data Group (PDG [4]) has listed the following isoscalar states with $J^{P C}=0^{++}$below a mass of $2 \mathrm{GeV}$ as "established":

$$
f_{0}(500), f_{0}(980), f_{0}(1370), f_{0}(1500), f_{0}(1710) \text {. }
$$

Special attention is paid to the reported evidence for $f_{0}(1370)$ which is the cornerstone for a particular scenario. This state comes along with $f_{0}(1500)$ nearby in mass, so they are discussed together. While attention is paid to data taken already long ago, we are witnessing now the advent of high precision results from $B$ factories as well as from the 
Large Hadron Collider and fixed target experiments at CERN with remarkable future potential.

There are some pros and cons for the different scenarios but finally it is hard to claim an ultimate evidence for the existence of a glueball. In the search for a direct evidence of gluonic mesons the leading clusters in gluon jets have been scrutinized in several LEP experiments. As a result, gluon jets are found different indeed from the expectations of Monte Carlo programs without glueballs, otherwise very successful (section 9).

Finally in section 10, we propose a new tool for identifying flavour multiplets based on symmetry relations for decay rates in heavy quarkonium decays, in particular in states $\chi_{c 0}, \chi_{c 2}$. Violation of such symmetries are indicative of glueball production. First available results are promising and should allow for further tests of the existing scenarios. The last section contains a summary and our preferred solution for scalar spectroscopy with glueball.

We focus here on questions related to glueball spectroscopy, so there is a large variety of experimental and theoretical works on the scalar sector which we do not address. There are recent reviews which include complementary material on various phenomenological and experimental issues [5] and on other theoretical [6] as well as experimental results [7].

\section{QCD expectations for glueballs}

\subsection{Quantum numbers}

In the first specific scenarios for glueball spectroscopy [2] it has been assumed that the counting of gluonic states is that obtained for free, massless gluons which have only two polarization states, so $n$ gluons can form $2^{n}$ different states. A simple case is met for the two-gluon channel. We consider the colour singlet states which correspond to the observable hadronic states. The colour averaged two-gluon systems carry the same quantum numbers as the two-photon systems. They have been studied by Landau [8] and Yang [9]; the general case is also addressed in a group theoretical analysis by Minkowski [10]. The results can be summarized as follows:

For a single photon there are two helicity states $\left|\lambda_{a}\right\rangle$ with $\lambda_{a}= \pm 1$ corresponding to the right and left circular polarization states. For two photons in the cms system we consider one photon in $+z$ direction, the other one in $-z$ direction. The states with two photons (likewise two gluons in a colour singlet state) have spin $J \neq 1$ ("Landau-Yang theorem") and $C$-parity $C=+1$. Four helicity states $\left|\lambda_{a} \lambda_{b}\right\rangle$ are available from which states of definite parity can be formed

$$
\begin{array}{cccccc}
(|11\rangle+|-1-1\rangle) ; & |1-1\rangle ; & |-11\rangle ; & (|11\rangle & - & |-1-1\rangle) \\
\leftarrow \rightarrow+\rightarrow \leftarrow & \rightarrow \rightarrow & \leftarrow \leftarrow & \leftarrow \rightarrow & - & \rightarrow \leftarrow
\end{array}
$$

the first three states have parity $P=+1$ the last one has $P=-1$. They form states of

definite $J^{P C}$ as can be found by studying the behaviour of these states under rotation 
and parity $P[9]$

$$
\left.0^{++}, 2^{++}, 4^{++} \ldots\right) ; 2^{++}, 4^{++} \ldots ; 3^{++}, 5^{++} \ldots ; 0^{-+},\left(2^{-+}, 4^{-+} \ldots\right.
$$

Here the states with $J^{P C}=2^{++}$and descendants correspond to the states $J_{z}= \pm 2$ above. Those states which are formed by "constituents" in a relative $S$ wave are expected to be lowest in mass and are enclosed by a box in (2); the higher spin excitations correspond to even orbital wave functions.

Three gluon systems can have both $C=+1$ and $C=-1$. For the ground states with relative $S$ waves one finds [2]

$$
\begin{array}{lll}
C=+1: & J^{P C}=1^{++} \\
C=-1: & J^{P C}=1^{+-}, 1^{--}, 3^{--} .
\end{array}
$$

\subsection{Lattice $Q C D$.}

Because of "asymptotic freedom" QCD interactions become weak for short distances where they can be analyzed within perturbation theory at small coupling between quarks and gluons. This method fails at large distances where the interactions become strong. Lattice QCD is a formulation of the theory on a space-time lattice and it is suitable for describing hadronic phenomena in the non-perturbative regime [11] (see [12] for an introduction and [13] for a recent review). Physical quantities can be computed from the QCD gauge and fermion actions $S_{G}$ and $S_{F}$ with the quark masses $m_{q}$ as parameters and a suitable mass scale.

The theory can be defined on a Euclidean space-time lattice with fermions on the lattice sites and gauge fields connecting the sites. The lattice spacing $a$ acts as an ultraviolet cutoff which provides a gauge invariant regularization of QCD. Physical observables can be obtained from an average over the relevant configurations of quark and gluon fields according to the Boltzmann weight $e^{-S_{F}-S_{G}}$ within a space time volume $L^{3} T$ and the physical results are obtained ultimately by extrapolation to the continuum limit $a \rightarrow 0$.

Hadron masses are calculated from 2-point correlation functions $C_{i j}(t)$ for operators $O_{i}$ relevant for the hadrons considered

$$
C_{i j}(t)=\frac{1}{Z} \int d \psi \int d \bar{\psi} \int d U e^{-S_{F}-S_{G}}\left\langle 0\left|O_{i}(t)^{\dagger} O_{j}(0)\right| 0\right\rangle
$$

in terms of a path integral over fermionic and gauge field variables $\psi, \bar{\psi}$ and $U$ with normalization $Z$. The correlation functions decrease exponentially for large Euclidean times $t$

$$
C_{i j}(t)=c_{i}^{m} c_{j}^{m} e^{-E_{m} t}
$$

where the term with the lowest energy $E_{0}$ dominates and determines the mass of the ground state. All masses are obtained in units of lattice spacing $a$ so that only ratios of masses are predicted. In order to relate to absolute scales a suitable physical mass scale 


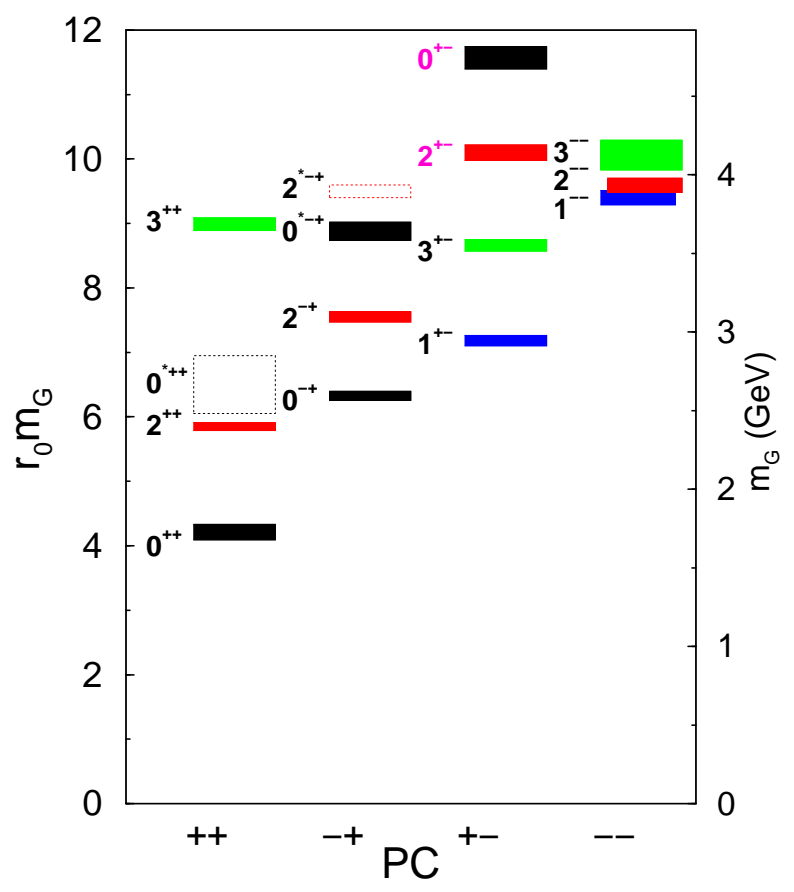

Figure 1. Mass spectrum of glueballs (in $\mathrm{GeV}$ on r.h.s.) for different quantum numbers $P C$ according to the quenched lattice calculations (figure from [18]).

has to be taken as input, such as the "string tension" or the "Sommer scale" $1 / r_{0} \sim 400$ $\mathrm{MeV}[14]$.

As examples of recent lattice calculations from first principles, we mention the results in full QCD on the conventional light hadron spectrum by the BudapestMarseilles-Wuppertal Collaboration [15] who has calculated the masses of the baryon octet and decuplet states as well as the masses of some light mesons within a few percent of accuracy. Here the masses of $\pi, K$ and $\Xi$ particles have been used to fix the masses of light and strange quarks at their physical values as well as the overall mass scale. Another result, obtained by the "Hadron Spectrum Collaboration" [16] concerns the spectrum of lightest and the first excited isoscalar meson states which includes quark-annihilation contributions. Remarkably, the mixing pattern of these mesons is reproduced close to observations.

More difficult to compute is the spectrum of glueballs in full QCD, as these states are heavier and therefore need higher statistics, in particular the scalar states with vacuum quantum numbers have extra contributions difficult to disentangle. In full QCD there is a mixing of gluonic and fermionic degrees of freedom, correspondingly one inserts gluonic and fermionic operators for the relevant correlation functions. For sufficiently light quark (pion) masses the glueball can decay into a meson pair which has to be included in the consideration as well.

The spectrum of glueballs has been calculated at first within the pure (Yang-Mills) gluon theory without quarks ("quenched approximation"). The lightest glueballs are 
found for $J^{P C}=0^{++}, 2^{++}$and $0^{-+}$which correspond to the $\mathrm{S}$ wave ground states of the three different spin configurations listed in (2). Masses have been obtained by several groups in good agreement within around $10 \%$ by Bali et al. [17], by Morningstar and Peardon [18] and with a similar calculation but for larger lattices and volumes by Chen et al. [19] at a lattice spacing of around $a=0.1-0.2 \mathrm{fm}$ (see Table 1). The results by Morningstar and Peardon [18] for states below $\sim 5 \mathrm{GeV}$ are shown in figure 1 . The three $S$ wave states above are indeed the states of lowest mass, the lightest states formed by three gluons with $J^{P C}=1^{+-}$and $1^{--}$for $C=-1$ are found at around $3 \mathrm{GeV}$ or higher.

Table 1. Glueball masses (in $\mathrm{MeV}$ ) in quenched lattice approximation; an additional error of $\sim 5 \%$ should be added for the scale error of $1 / r_{0}=410(20) \mathrm{MeV}$ [18].

\begin{tabular}{llll}
\hline$J^{P C}$ & Bali et al. [17] & Morningstar et al. [18] & Chen et al. [19] \\
\hline $0^{++}$ & $1550(50)$ & $1730(50)$ & $1709(49)$ \\
$2^{++}$ & $2270(100)$ & $2400(25)$ & $2388(23)$ \\
$0^{-+}$ & $2330(260)$ & $2590(40)$ & $2557(25)$ \\
\hline
\end{tabular}

The influence of dynamical $q \bar{q}$ contributions in full QCD on the mass of flavour singlet gluonic mesons has been studied by Hart and Teper (UKQCD collaboration [20]). In this exploratory investigation the lattice spacing was around $a \sim 0.1 \mathrm{fm}$ and the light quark masses about one half the strange quark mass; an extrapolation to physical limits was not possible. A significant suppression of the scalar glueball mass by the amount $\sim 0.84 \pm 0.03$ with respect to the quenched value was found. With increasing quark mass this suppression should disappear and pure gluo-dynamics be restored. As this effect was not observed, not even for quark masses twice the strange quark mass, no real significance has been attributed to this suppression effect. Furthermore, the spin 2 glueball did not show any substantial deviation from the quenched result.

A different conclusion has been reached by Hart et al. (UKQCD [21]) within a study using both gluonic and fermionic operators to create the flavour singlet states. There have been $N_{f}=2$ degenerate sea-quarks using a sample of a total of $\sim 1500$ configurations. At comparable lattice spacing the mass of the lightest gluonic meson is reduced considerably with respect to the unquenched result from $1600 \mathrm{MeV}$ to about $1000 \mathrm{MeV}$. The results on the two lightest $0^{++}$mesons are interpreted in terms of a maximal mixing of the $\bar{q} q$ and $g g$ states into the physical flavour singlet mesons around 1000 and $1600 \mathrm{MeV}$.

More recently, a study with higher statistics and including strange quarks has been presented by Richards et al. (UKQCD [22]). They use measurements at pion masses of 280 and $360 \mathrm{MeV}$ and a spacing of $a=0.123 \mathrm{fm}$ and $a=0.092 \mathrm{fm}$ with 3000 and 5000 configurations respectively. In their analysis gluonic and $\pi \pi$ operators have been included but no fermionic operators. This approach did not show any essential difference between unquenched and quenched results for the lightest $0^{++}$state. A mixing of the glueball with the $\bar{q} q$ state as found in [21] has not been considered explicitly. 
Similarly, for the $2^{++}$and $0^{-+}$states the results on the masses show little difference between quenched and unquenched calculations and the same conclusion has been drawn by the same collaboration (UKQCD) for glueballs of yet higher mass [23]. A summary of the unquenched calculations is presented in Table 2. It should be noted that the scale parameter $r_{0}$ is different in quenched and unquenched calculations, but the values actually used in [18] and [22] differ by $<3 \%$.

Table 2. Glueball masses from lattice QCD: listed are the ratios of masses from unquenched over quenched calculations.

\begin{tabular}{llll}
\hline$J^{P C}$ & Hart and Teper [20] & Hart et al. [21] & Richards et al. $[22]$ \\
\hline $0^{++}$ & $0.84(3)$ & 0.63 & $1.03(3)$ \\
$2^{++}$ & $1.03(3)$ & & $0.98(10)$ \\
$0^{-+}$ & & & $0.97(2)$ \\
\hline
\end{tabular}

For the future studies a yet higher accuracy of the simulations is suggested in [22], also smaller quark masses should be used to allow for a more realistic decay of the glueball into two pions. Furthermore it is argued that a definitive calculation required a continuum extrapolation, and the inclusion of fermionic operators [23]. In that way the mixing problem between $g g, \bar{q} q$ and $\pi \pi$ states could ultimately be clarified.

\subsection{QCD sum rules}

An alternative approach to obtain information on the hadronic spectrum within QCD and on the glueball properties in particular is based on the "QCD sum rules" [24] (see [25] for a pedagogical presentation and [26] for a recent review). It starts from a correlation function in Minkowski space involving gluonic or fermionic operators $O_{i}$ according to the considered $J^{P C}$ quantum numbers which is evaluated in the deeply space like region

$$
\Pi\left(Q^{2}\right)=i \int d^{4} x e^{i q x}\left\langle 0\left|T\left\{O_{1}(x) O_{2}(0)\right\}\right| 0\right\rangle \quad\left(Q^{2} \equiv-q^{2} \gg \Lambda_{Q C D}^{2}\right) .
$$

In an application of the operator product expansion the short distance contribution to the correlation function is calculated from QCD perturbation theory whereas the long distance contribution is given in terms of "vacuum condensates". These parameters, ultimately, have to be determined from experiment, of special importance are the condensates of lowest dimension, the quark and gluon condensates $\langle\bar{q} q\rangle$ and $\left\langle\frac{\alpha_{s}}{\pi} G_{\mu \nu}^{a} G_{\mu \nu}^{a}\right\rangle$. One can estimate the low energy hadronic spectrum by matching the correlator (5), as given by this expansion, with a dispersion relation of the type

$$
\Pi\left(Q^{2}\right)=\frac{1}{\pi} \int_{0}^{\infty} d s \frac{\operatorname{Im} \Pi(s)}{\left(s+Q^{2}\right)}
$$

in general, (6) has to be modified by subtractions depending on the high energy behaviour of the absorptive part $\rho(s) \equiv \operatorname{Im} \Pi(s)$ which adds terms with powers of $Q^{2}$ 
in (6). The absorptive part can be represented as a sum over the low lying hadronic states and a continuum contribution, in the simplest case of one resonance as

$$
\rho(s)=\rho^{r e s}(s)+\theta\left(s-s_{0}\right) \operatorname{Im} \Pi^{Q C D}(s), \quad \frac{1}{\pi} \rho^{r e s}=f_{1}^{2} \delta\left(s-m_{r e s}^{2}\right),
$$

where $s_{0}$ denotes the threshold of the QCD continuum. For better sensitivity to low $Q^{2}$ of (6) one performs a "Borel transformation" which calculates the integral

$$
L^{(k)}(\tau)=\frac{1}{\pi} \int_{0}^{\infty} d t t^{k} e^{-t \tau} \operatorname{Im} \Pi(t)
$$

with index $k=-1,0,1, \ldots$. In this way a set of sum rules for index $k$ is obtained.

The successful application of this formalism to a large variety of hadronic phenomena concerning conventional mesons and baryons has given a good confidence to this approach besides providing the necessary phenomenological parameters. Consequently, this scheme has been applied to the analysis of glueballs as well.

It has been suggested that the valence gluons bound in a glueball couple much stronger to the vacuum fields, than, for example, the quarks in the $\rho$ meson where a description in terms of mean vacuum fields is sufficient [27]. Ultimately, this strong coupling to vacuum fields leads to the higher mass scales for glueballs. Particularly strong effects from vacuum fields occur in spin zero systems of low energy, a problem which has been approached by the inclusion of instanton field configurations. Along this line of thought the radius of the $0^{++}$glueball is only $0.2 \mathrm{fm}$ to be compared with $0.5 \mathrm{fm}$ for the $\rho$ meson $[28,25]$.

The analysis of sum rules with lowest indices $k=-1,0$ (referred to as "subtracted" [27] and "unsubtracted" [29] sum rules) by Narison and Veneziano [30] leads to a consistent solution including two gluonic scalar states with masses (see [31] for an update)

$$
M_{g b 1} \sim 0.9-1.1 \mathrm{GeV}, \quad M_{g b 2} \sim 1.5-1.6 \mathrm{GeV}
$$

Furthermore, for the state around $1 \mathrm{GeV}$, the application of certain low energy sum rules predict a large width into $\pi \pi$ with $[30,31]$

$$
\Gamma\left(M_{g b 1 \rightarrow \pi^{+} \pi^{-}}\right) \sim 0.7 \mathrm{GeV}
$$

In an extension of this result a phenomenological scheme has been suggested for $f_{0}(500-1000) / \sigma \neq, f_{0}(980)$ and a sequence of isoscalar mesons heavier than $1 \mathrm{GeV}$ realizing a mixing of $q \bar{q}$ and gluonic states as in (9) and a radial gluonic excitation [32].

An approach emphasizing the role of instantons by Forkel [33] suggests a solution of sum rules with a single scalar state at a mass of $M_{g b}\left(0^{++}\right) \sim 1.25 \pm 0.2 \mathrm{GeV}$, in between the previous results (9). The same approach has also been applied to the pseudoscalar gluonium and gave the result $M_{g b}\left(0^{-+}\right)=2.2 \pm 0.2 \mathrm{GeV}$, similar to the lattice predictions mentioned above.

$\ddagger$ The higher mass $1000 \mathrm{MeV}$ refers to the "Breit-Wigner" or "on-shell" mass which are more appropriate for sum rules (see section 5.1.1). 
More recently, a sum rule study of the mixed scalar system of gluonium $(g g)$ and quarkonium $(q \bar{q})$ has been presented by Harnett et al. [34] which also included instanton contributions. The analysis of the diagonal $q \bar{q}$ and $g g$ as well as the non-diagonal $q g$ sum rules yields a consistent solution when using two mass states in (7) at approximately $1 \mathrm{GeV}$ and $1.4 \mathrm{GeV}$, similar to (9). In this solution the couplings of the two hadronic states to $q \bar{q}$ and $g g$ "constituents" is comparable, corresponding to a near maximal mixing, with a slight preference of the heavier state to couple to $g g$. This would imply that there is only one glueball and one quark-antiquark state in this scheme for the two hadronic states. The heavier mass state could be related to $f_{0}(1500)$ and the lower mass state to either $f_{0}(500-1000) / \sigma$ or $f_{0}(980)$, both higher and lower mass states being mixed of $g g$ and $q \bar{q}$.

\subsection{Conclusions from theory}

There is a general agreement in that the lightest gluonic state has quantum numbers $J^{P C}=0^{++}$. One state is located around 1.4-1.7 GeV. A second state is suggested in sum rules and in certain unquenched lattice calculations near $1 \mathrm{GeV}$. In these analyses there is a strong, almost maximal mixing of the hadronic states near 1 and $1.6 \mathrm{GeV}$ into the $q \bar{q}$ and $g g$ components. This agreement between both approaches is striking but not universally accepted. In particular, the most recent lattice calculation does not find this low mass state, but, given the computational limitations, its existence cannot be excluded either. A definitive calculation requires a higher statistics, a continuum extrapolation and the inclusion of fermionic operators.

The next heavier states are expected with quantum numbers $J^{P C}=0^{-+}, 2^{++}$and with masses $\gtrsim 2 \mathrm{GeV}$. States with other quantum numbers including exotic, i.e. non- $q \bar{q}$ ones, are expected for higher masses. Experimental analyses have been difficult so far in this mass region. Therefore, the search for the scalar gluonic states looks particularly promising despite the experimental and theoretical uncertainties.

\section{Experimental search strategies for glueballs}

In the following we will mainly focus on the scalar glueball which is expected to be the lightest one and which has been addressed in most research studies. The isoscalar states with $J^{P C}=0^{++}$listed as "established" by the PDG [4] below a mass of $2 \mathrm{GeV}$ are already listed in (1). All these states have been considered as glueball candidates or mixed glueball/quarkonium states in some of the models or theories. First we discuss some conventional strategies for distinguishing glueballs.

\subsection{Quark-gluon constituent structure of hadrons from their decays}

The 2-body strong decays of an unstable hadron reflect its constituent structure. Under $S U(3)_{f l}$ symmetry of the strong interactions a light quarkonic meson $M\left(q \bar{q}^{\prime}\right)$ decays by 
creation of quark anti-quark pairs from the vacuum with equal amplitudes

$$
|0\rangle \rightarrow|u \bar{u}\rangle+|d \bar{d}\rangle+|s \bar{s}\rangle .
$$

into a superposition of meson states $M\left(q \bar{q}_{i}\right) M\left(q_{i} \bar{q}^{\prime}\right)$. A glueball can decay by creating $q \bar{q}$ pairs and form quarkonic mesons or by gluons and form gluonic mesons. An explicit model for such decays has been proposed in [35] and a similar approach in [36, 37] with small differences for glueballs. The role of OZI suppressed processes has been studied by Zhao [38].

The flavour symmetry can be broken in different ways.

- Strange quark suppression: because of the heavier strange quark mass the ratio

$$
\rho \equiv\langle s \bar{s}|V| 0\rangle /\langle d \bar{d}|V| 0\rangle
$$

of matrix elements for the creation of $s \bar{s}$ versus $u \bar{u}$ or $d \bar{d}$ is suppressed, i.e. $\rho<1$. Empirically, $\rho \geq 0.8$ for established nonets, for tensor mesons $\rho=0.96 \pm 0.04$ is found in [35] using form factors, in [36] $\rho=0.7-0.9$ without form factors.

- Form factors: they may provide momentum dependent effects, representing, for example, inelastic channels at higher momenta or the influence of angular momentum barriers. In [35] the choice is

$$
\left|F_{i j}(\vec{q})\right|^{2}=\exp \left(-q^{2} / 8 \beta^{2}\right)
$$

which multiplies the phase space and threshold factor

$$
S_{p}(q)=q^{2 \ell+1}
$$

for angular momentum $\ell$ and momentum $q=|\vec{q}|$ with $\beta=0.4 \mathrm{GeV}$.

- Chiral effects: For the decay of a scalar glueball $G$ the amplitude $M(G \rightarrow q \bar{q}) \propto m_{q}$ (quark mass $m_{q}$ ) according to a perturbative analysis using chiral symmetry which involves the intermediate process $g g \rightarrow q \bar{q}$ [39]. In consequence, the decay branching ratio $r_{K \pi}=B\left(G \rightarrow K^{+} K^{-}\right) / B\left(G \rightarrow \pi^{+} \pi^{-}\right)$is enhanced over $r_{K \pi}=1$ for flavour symmetry, whereas strange quark suppression would lead to $r_{K \pi}<1$.

3.1.1. Quarkonium $(q \bar{q})$ decays. In our comparison with glueball decays we consider here mainly isoscalar quarkonium states $|q \bar{q}\rangle$ which are generally mixed from non-strange $|n \bar{n}\rangle=\frac{1}{\sqrt{2}}|u \bar{u}+d \bar{d}\rangle$ and strange quark $|s \bar{s}\rangle$ components

$$
|q \bar{q}\rangle=\cos \alpha|n \bar{n}\rangle-\sin \alpha|s \bar{s}\rangle .
$$

Alternatively, the mixing can be described in terms of the $S U(3)_{f l}$ singlet and octet components $|1\rangle=\frac{1}{\sqrt{3}}|u \bar{u}+d \bar{d}+s \bar{s}\rangle$ and $|8\rangle=\frac{1}{\sqrt{6}}|u \bar{u}+d \bar{d}-2 u \bar{u}\rangle$. The mixing angle $\alpha$ in the quark-flavour basis is related to the nonet mixing angle $\theta$ in the singlet-octet basis by

$$
\alpha=\theta+\arctan \sqrt{2} \simeq \theta+54.7^{\circ} .
$$


For $\alpha=0(\pi / 2)$ we obtain pure $n \bar{n}(s \bar{s})$ states ("ideal mixing") as approximately found for the vector mesons $\rho, \omega, \phi$. The mixing of pseudoscalar mesons is written as

$$
\begin{aligned}
& |\eta\rangle=\cos \phi_{p s}|n \bar{n}\rangle-\sin \phi_{p s}|s \bar{s}\rangle, \\
& \left|\eta^{\prime}\right\rangle=\sin \phi_{p s}|n \bar{n}\rangle+\cos \phi_{p s}|s \bar{s}\rangle .
\end{aligned}
$$

According to a recent review [40], the different analyses from radiative decays of vector and pseudoscalar mesons yield values $\phi_{p s} \approx 42^{\circ}\left(\theta_{p s} \simeq-13^{\circ}\right)$, not far away from the pure flavour singlet-octet states $\left(\theta=0,-\pi / 2\right.$ or $\left.\phi_{p s}=54.7^{\circ},-35.3^{\circ} \ddagger\right)$. On the other hand, the possibility of a gluonic component of $\eta^{\prime}$ remains ambivalent. Such a component can be added to $\eta^{\prime}$, for example, by introducing an additional mixing angle $\phi_{G p s}$

$$
\left|\eta^{\prime}\right\rangle=\cos \phi_{G p s}\left(\sin \phi_{p s}|n \bar{n}\rangle+\cos \phi_{p s}|s \bar{s}\rangle\right)+\sin \phi_{G p s}|g g\rangle
$$

For the last term with $Z_{\eta^{\prime}}=\sin \phi_{G p s}$ one finds typically $\left|Z_{\eta^{\prime}}\right|^{2} \lesssim 0.1$ but larger values up to $\left|Z_{\eta^{\prime}}\right|^{2} \lesssim 0.5$ are reported as well [40]. A more general approach with two mixing angles describing the mixing of meson states at the hadronic scale and also the mixing of decay constants at short distances is discussed in [41, 42]. Our analysis of $\chi_{c}$ decays in the next subsection suggests that the conventional state mixing approach as above is appropriate in that case.

According to the model [35] the partial width $\Gamma_{i j}$ of a quarkonium state into a pair of mesons $M_{i} M_{j}$ is computed from

$$
\Gamma_{i j}=\gamma_{i j}^{2} \times\left|F_{i j}(\vec{q})\right|^{2} \times S_{p}(\vec{q})
$$

with $(13),(14)$; it depends on the above parameters $\alpha$ and $\rho$ through the couplings

$$
\gamma_{i j}^{2}=c_{i j}\left|M_{i j}\right|^{2}
$$

where $M_{i j}$ is the decay amplitude and $c_{i j}$ is a weighting factor for the different charge states. For an isoscalar $(q \bar{q})$ decay these couplings $\gamma_{i j}^{2}$ are given in table 3 for $r_{G}=0$ $[35,36]$; the dependence of $\gamma_{i j}^{2}$ on $\alpha$ is shown in figure 2 .

3.1.2. Glueball decays. Bound gg states ("gluonia") decay through a two gluon intermediate state where several mechanisms can be envisaged [35] (see figure 3)

$$
\begin{array}{rlrl}
\text { a) } & g b & \rightarrow g g \rightarrow \bar{q} q \bar{q}^{\prime} q^{\prime} & \rightarrow M M^{\prime} \\
\text { b) } \quad g b \rightarrow g g & \rightarrow g g g g & \rightarrow \eta\left(\eta^{\prime}\right) \eta\left(\eta^{\prime}\right) \quad \text { or } \quad \rightarrow g b g b \\
\text { c) } \quad g b & \rightarrow g g \rightarrow \bar{q} q \rightarrow \bar{q} q \bar{q}^{\prime} q^{\prime} \rightarrow M M^{\prime}
\end{array}
$$

In the decay rates of these processes the ratio of amplitudes

$$
R \equiv\langle s \bar{s}|V| g\rangle /\langle d \bar{d}|V| g\rangle
$$

$\ddagger$ Previously, a favoured value was quoted as $\phi_{p s}=37.4^{\circ}, \theta_{p s}=-17.3^{\circ}[3]$. 


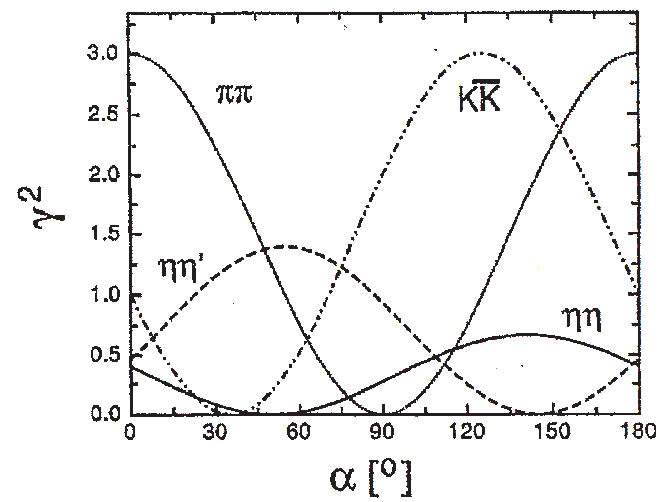

Figure 2. Couplings $\gamma_{i j}^{2}$ for isoscalar $(q \bar{q})$ meson decays as function of mixing angle $\alpha$ as in (15) for $S U(3)_{f l}$ symmetry $(\rho=1)$ from table 3 with $r_{G}=0$ for $\phi_{p s}=37.4^{\circ}$ (figure from [35]).

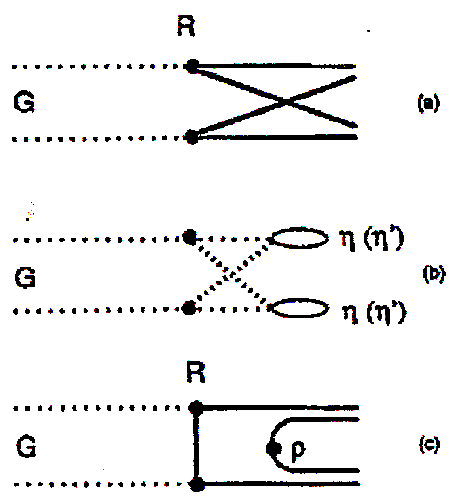

Figure 3. Processes contributing to gluonium decay (21a-21c) (figure from [35]).

Table 3. Couplings $\gamma_{i j}^{2}$ for decay of an $I=0$ meson $f_{0}$ with mixed $(q \bar{q})$ and $(g g)$ components [35, 36]; flavour mixing angles $\alpha$ as in $(15), \phi \equiv \phi_{p s}$ as in (17a); glueball decay according to $(21 a)[35] ; S U(3)_{f l}$ breaking parameters $\rho, R$ as defined in (12) and (22); weights $c_{i j}$ included in $\gamma_{i j}^{2}$; decay couplings $g_{i j}^{2}=g^{2} \gamma_{i j}^{2} / 4$ for $(q \bar{q})$ and $r_{G}=\sqrt{3 /\left(2+R^{2}\right)} G / g$ for $(g g)$ with $g, G$ from (24) (based on [36, 37]).

\begin{tabular}{ccc}
\hline decay channel & couplings $\gamma_{i j}^{2}$ for $\left|f_{0}\right\rangle=\cos \phi_{G}|q \bar{q}\rangle+\sin \phi_{G}|g g\rangle$ & weights \\
\hline$\pi \pi$ & $3\left(\cos \alpha+r_{G}\right)^{2}$ & 3 \\
$K \bar{K}$ & $\left(\rho \cos \alpha-\sqrt{2} \sin \alpha+2 r_{G}\right)^{2}$ & 4 \\
$\eta \eta$ & $\left(\cos \alpha \cos ^{2} \phi-\sqrt{2} \rho \sin \alpha \sin ^{2} \phi+r_{G}\left(\cos ^{2} \phi+R^{2} \sin ^{2} \phi\right)\right)^{2}$ & 1 \\
$\eta \eta^{\prime}$ & $2 \cos ^{2} \phi \sin ^{2} \phi\left(\cos \alpha+\sqrt{2} \rho \sin \alpha+r_{G}\left(1-R^{2}\right)\right)^{2}$ & 2 \\
$\eta^{\prime} \eta^{\prime}$ & $\left(\cos \alpha \sin ^{2} \phi-\sqrt{2} \rho \sin \alpha \cos ^{2} \phi+r_{G}\left(\sin ^{2} \phi+R^{2} \cos ^{2} \phi\right)\right)^{2}$ & 1 \\
\hline
\end{tabular}


appears for the $q \bar{q}$ production from a gluon, where $R^{2}=1$ for $S U(3)_{f l}$ symmetry. For the decay of a mixed state

$$
\left|f_{0}\right\rangle=\cos \phi_{G}|q \bar{q}\rangle+\sin \phi_{G}|g g\rangle
$$

with $|q \bar{q}\rangle$ mixed as in (15) and $|g g\rangle$ decaying as in (21a) into pairs of pseudoscalars we write the decay couplings as [37]

$$
g_{i j}=g d_{i j}^{q \bar{q}}+G d_{i j}^{g g} ; \quad g=g_{0} \cos \phi_{G}, \quad G=G_{0} \sin \phi_{G} .
$$

The couplings $\gamma_{i j}^{2}$ for the decay of the mixed state with the factor $g^{2} / 4$ taken out and with $r_{G}=\sqrt{3 /\left(2+R^{2}\right)} G / g$ are given in table 3. For the glueball decay with flavour symmetry $(R=1)$ one finds from the term with $r_{G} \propto G$ the reduced partial widths $\gamma_{i j}^{2}$

$$
\gamma_{i j}^{2}\left(g b \rightarrow \pi \pi: K \bar{K}: \eta \eta: \eta \eta^{\prime}: \eta^{\prime} \eta^{\prime}\right)=3: 4: 1: 0: 1
$$

according to the statistical weights.

The two-gluon intermediate process also dominates the decays of scalar heavy quark, $c \bar{c}$, bound states with $C$ parity $C=+1$ like $\chi_{c}$ in the perturbative analysis and one expects decay rates as in (25). An update of this test for spin $J=0,2$ charmonia $\chi_{c 0}, \chi_{c 2}[35]$ is carried out in table 4 . We list the measured branching ratios $B$ into pairs of pseudoscalars according to the PDG [4], where recent contributions come from CLEO [43] and BES [44]. From the model (but without dynamical formfactor $F_{i j}\left(q^{2}\right)$, i.e. $\beta \rightarrow \infty)$ one expects $B \propto c|M|^{2} q^{2 \ell+1}$. In table 4 we also show $B$ after division by $c q^{2 \ell+1}$, and after normalization to that value for the $\pi \pi$ decay. These data are to be compared with the model predictions for $|M|^{2}=\gamma^{2} / c$ in table 3 for $(g g)$, i.e. for $g / G \rightarrow 0$, where recall $g$ and $G$ are given by (24). These expectations are given in table 4 as well for different values of the strange quark parameter $R$ in (22). We observe, that the expectations from $S U(3)_{f l}$ symmetry $(R=1)$ are fulfilled for all decay processes of $\chi_{c 0,2}$ within $10-20 \%$ (only the upper bound for $\chi_{c 2} \rightarrow \eta^{\prime} \eta^{\prime}$ is a bit low). So there is no strong need for the additional gluonic processes $(21 b)$ affecting $\eta, \eta^{\prime}$; nor is there any strong need for dynamical form factors $F_{i j}\left(q^{2}\right)$; the weakness of angular momentum barrier factors (of Blatt-Weisskopf type) may be due to the small radius of charmonium states of $O\left(1 / m_{c}\right)$.

Looking at the substructures at the $10 \%$ level we note that for the tensor state $\chi_{c 2}$ the observed values for $B /\left(c q^{5}\right)$ would prefer a slightly reduced $R \gtrsim 0.9$. In case of the decays of the scalar $\chi_{c 0}$ values of $B /(c q)$ for kaons are slightly enhanced by 10$20 \%$. This effect could be a remote consequence of the chiral enhancement in scalar decays discussed above from the contribution of the intermediate process $g g \rightarrow q \bar{q}$ as considered for scalar glueballs in (21c) (the argument is also applicable for a decaying scalar $c \bar{c}$ state). The effect can be simulated by choosing $R>1$ and we give in the last line of table 4 the predictions for $R=1.1$ which would fit the data for $\chi_{c 0}$ generally better.

A modification of the $S U(3)_{f l}$ symmetry for decay rates (with $R=1$ in table 4 ) is expected in schemes which include state mixing at hadronic scales and mixing of decay 
Table 4. Branching ratios $B$ of $\chi_{c 0}(3415)\left(0^{++}\right)$and $\chi_{c 2}(3556)\left(2^{++}\right)$into pairs of pseudoscalars; also $B /(c q)$ or $B /\left(c q^{5}\right)$ resp. with charge weight $c$ and momentum $q$ after normalization to the value for $\pi \pi$ (data as listed in [4]), together with the resp. theoretical expectations $|M|^{2}=\gamma_{i j}^{2} / c$ for process $g g$ in table 3 for different strange quark parameters $R$ (mixing angle $\phi_{p s} \approx 37.4^{\circ}$ for $\eta, \eta^{\prime}$ ).

\begin{tabular}{lcccccc}
\hline & $\pi \pi$ & $K^{+} K^{-}$ & $K_{s}^{0} K_{s}^{0}$ & $\eta \eta$ & $\eta \eta^{\prime}$ & $\eta^{\prime} \eta^{\prime}$ \\
weight $c$ & 3 & 2 & 1 & 1 & 2 & 1 \\
\hline$\chi_{c 0}\left(0^{++}\right)$ & & & & & & \\
$B \times 10^{3}$ & $8.5 \pm 0.4$ & $6.06 \pm 0.35$ & $3.14 \pm 0.18$ & $3.03 \pm 0.21$ & $<0.24$ & $2.02 \pm 0.22$ \\
$B /(c q)$ & $1 . \pm 0.05$ & $1.13 \pm 0.06$ & $1.17 \pm 0.07$ & $1.14 \pm 0.08$ & $<0.048$ & $0.87 \pm 0.09$ \\
\hline$\chi_{c 2}\left(2^{++}\right)$ & & & & & & \\
$B \times 10^{3}$ & $2.43 \pm 0.13$ & $1.09 \pm 0.08$ & $0.58 \pm 0.05$ & $0.59 \pm 0.05$ & $<0.06$ & $<0.11$ \\
$B /\left(c q^{5}\right)$ & $1 . \pm 0.06$ & $0.82 \pm 0.06$ & $0.88 \pm 0.08$ & $0.94 \pm 0.08$ & $<0.063$ & $<0.32$ \\
\hline expect & & & & & & \\
$\mathrm{R}=1.0$ & 1. & 1. & 1. & 1. & 0. & 1. \\
$\mathrm{R}=0.95$ & 1. & 0.9 & 0.9 & 0.93 & 0.002 & 0.88 \\
$\mathrm{R}=0.90$ & 1. & 0.81 & 0.81 & 0.86 & 0.008 & 0.77 \\
$\mathrm{R}=1.1$ & 1. & 1.1 & 1.1 & 1.08 & 0.002 & 1.13 \\
\hline
\end{tabular}

constants at short distances [42]; in that case the ratios of the $\chi_{c}$ decay rates deviate considerably from unity, for example $B\left(\chi_{c 0} \rightarrow \eta \eta\right) / B\left(\chi_{c 0} \rightarrow \pi^{0} \pi^{0}\right)=1.9$ and the same follows for the ratio $\left(\eta^{\prime} \eta^{\prime}\right) /\left(\pi^{0} \pi^{0}\right)$. The data appear to be closer to the conventional meson state mixing approach as seen in table 4 .

The remarkable success of the simple scheme based on $S U(3)_{f l}$ symmetry with phase space correction for decays into the same nonets suggests further tests with other nonets in the search for extra gluonic states, as will be discussed in section 10.

\subsection{Enhanced and suppressed glueball production}

Not only the decay, also the production properties are characteristic for the intrinsic structure of a hadronic state $[45,46]$.

3.2.1. Gluon-rich processes. There are processes which provide a "gluon rich" environment with enhanced probability for glueball production, see figure 4 for examples. These processes have been extensively explored experimentally in the past $[5,7]$. We give here a survey first over different processes and come back to specific problems later:

(i) Radiative $J / \psi$ or $\Upsilon$ decay: In the perturbative approach the $(c \bar{c})$ or other heavy quarkonium $J^{P C}=1^{--}$bound states decay predominantly through $(Q \bar{Q}) \rightarrow 3 g$. Alternatively, the radiative decay $(Q \bar{Q}) \rightarrow \gamma+(2 g)$ is possible with formation of an intermediate gluonium $(g g)$ state $((Q \bar{Q}) \rightarrow \gamma+g b)$. 
$(1)$

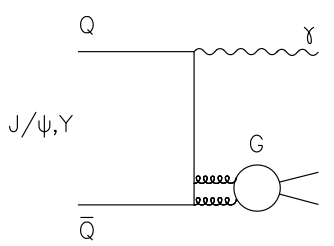

(II)

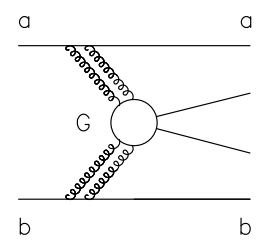

(III)

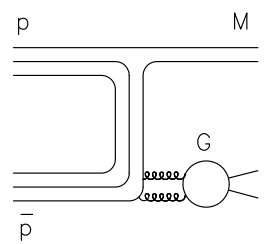

(IV)

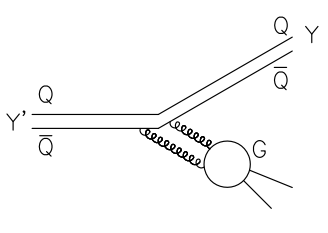

Figure 4. Processes favouring glueball $G$ production, where $J / \psi$ and $\Upsilon$ are respectively the lowest mass $c \bar{c}$ and $b \bar{b}$ mesons with $J^{P C}=1^{--}$.

(ii) Central production of mesons: In double diffractive high energy processes the incoming hadrons scatter with small momentum transfers and carry on the initial valence quarks. In Regge theory this process is dominated by "double Pomeron exchange". If the Pomeron is viewed as a gluon dominated object then glueball production is enhanced in this reaction $(p p \rightarrow p g b p)$. The different contributing processes within QCD have been discussed in [47].

(iii) $p \bar{p}$ annihilation: The annihilation of quarks may proceed through intermediate gluons and the formation of glueballs $(p \bar{p} \rightarrow g b+M)$.

(iv) Decay of excited heavy quarkonium $Y^{(n)}$ to ground state $Y$ : In the example $Y^{(n)} \rightarrow Y+X$ the hadrons $X$ are emitted from intermediate gluons and therefore could be formed through an intermediate glueball.

(v) Decay of heavy quark $b \rightarrow s g$ : This QCD process (through "penguin" diagram) may hadronize involving a glueball according to $B \rightarrow K g b$ [48].

(vi) Leading particle in gluon jet: In analogy to the fragmentation of the primary quark $q$ of a $q$-jet into an energetic meson $M\left(q \bar{q}^{\prime}\right)$ which carries $q$ as valence quark, there may be the fragmentation of the primary gluon of a gluon jet into an energetic meson $M(g g)$ which carries the initial gluon as valence gluon $g \rightarrow g b+X$ (section 9 ).

3.2.2. Suppression of glueballs in $\gamma \gamma$ processes. Having neutral constituents a glueball couples to photons only through loop processes and then it is suppressed in $\gamma \gamma$ reactions.

\subsection{Supernumerous states among q $\bar{q}$ nonets?}

Mesons with light quark constituents ( $u, d, s$ quarks) are classified in nonets of $3 \times 3$ states (octet+singlet). A well known example is the pseudoscalar nonet of lowest mass with $\pi, K, \eta$ near flavour octet and $\eta^{\prime}$ near singlet. It is the aim of meson spectroscopy to establish the appropriate classification of mesons. They should fit into nonets of $q \bar{q}$ states - possibly, there are also exotic states like tetra-quark $q q \bar{q} \bar{q}$ or hybrid $q \bar{q} g$ states. If there are glueballs in addition there should be supernumerous states which do not fit into a nonet classification of the meson spectrum. 


\section{Spectroscopy of scalar mesons: $q \bar{q}$ nonets mixing with glueballs}

In the scalar sector different schemes have been proposed for a spectroscopy with glueballs for the mesons below $2 \mathrm{GeV}$ including the isoscalars of (1).

Table 5. Spectroscopy with glueballs, route 1: glueball near $1500 \mathrm{MeV}$.

\begin{tabular}{cccc}
\hline$I=0$ & $I=\frac{1}{2}$ & $I=1$ \\
\hline & $K^{*}(1950)$ & \\
\hline$f_{0}(1710)$ & & & 3 isoscalars: $q \bar{q}$ nonet \\
$f_{0}(1500)$ & $K^{*}(1430)$ & $a_{0}(1450)$ & + glueball $\mathrm{M} \sim 1.6 \mathrm{GeV}$ \\
$f_{0}(1370) ?$ & & & \\
\hline$f_{0}(980)$ & & $a_{0}(980)$ & light nonet \\
& $K^{*}(900) / \kappa ?$ & & $q \bar{q}$ or $q q-\bar{q} \bar{q}$ \\
$f_{0}(500) / \sigma$ & & & or $K \bar{K}$ \\
\hline
\end{tabular}

\subsection{Route 1: $(q \bar{q})$ nonet - glueball mixing around $1500 \mathrm{MeV}$}

Lattice QCD in quenched approximation predicts the lightest glueball in the mass range around $1600 \mathrm{MeV}$ (see section 2.2). The discovery of the rather narrow $f_{0}(1500)$ in $p \bar{p}$ annihilation by the Crystal Barrel Collaboration [49] and by GAMS [50] suggested at first the possibility of a gluonic state. A closer inspection of decay mechanisms and branching ratios led Amsler and Close [35] to propose a mixing scheme including $f_{0}(1370)$ and $f_{0}(1710)$ with a gluonic component as well $\S$. In table 5 we list the meson states ordered according to mass and isospin. Between 1.0 and $1.9 \mathrm{GeV}$ we find the states fitting into a $q \bar{q}$ nonet but with one supernumerous isoscalar which suggests the presence of a glueball.

With more experimental results available and with further development of models various mixing schemes for the three isoscalars have been proposed (for a further discussion, see [7]). As an example, we present the result [51] which includes data for $\gamma \gamma$ processes. The physical mass states $f_{0}\left(m_{i}\right)$ are decomposed into the $q \bar{q}$ and $g g$ states or, alternatively, into the $S U(3)$ eigenstates $|1\rangle=(|u \bar{u}\rangle+|d \bar{d}\rangle+|s \bar{s}\rangle) / \sqrt{3}$ and $|8\rangle=(|u \bar{u}\rangle+|d \bar{d}\rangle-2|s \bar{s}\rangle) / \sqrt{6}$ and gluonium $|g g\rangle$

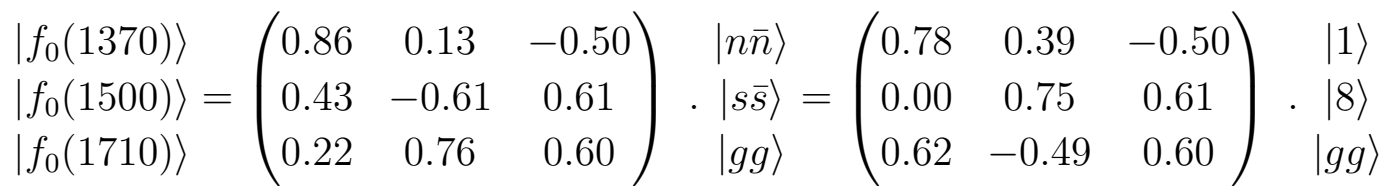

One observes that in this scheme $f_{0}(1500)$ is a mixture only of octet $q \bar{q}$ and glueball; the glueball component is distributed over all three $f_{0}$ states (last column in mixing matrix) with about equal amounts. In alternative schemes either $f_{0}(1500)$ can be dominantly gluonic [52] or $f_{0}(1710)$ while $f_{0}(1370)$ is near singlet $[53,52]$.

$\S$ In the scheme [35] the existence of the heavy state (now $f_{0}(1710)$ ) actually has been predicted. 
A possible problem with this mixing scheme are the doubts related to the very existence of $f_{0}(1370)$. This resonance is included in global fits of parametric model amplitudes to a variety of channels but it is not seen in any model independent bin-bybin phase shift analysis. The PDG under this entry does not provide any established result on branching ratios or ratios thereof - contrary to the well established nearby $f_{0}(1500)$. We come back to this problem in more detail in section 7 .

The light scalar mesons below $1 \mathrm{GeV}$ can be grouped into another nonet (see table 5) which includes the broad states $f_{0}(500) / \sigma$ and $K^{*}(800) / \kappa$, besides the narrow $f_{0}(980)$ and $a_{0}(980)$. This meson nonet can been built from $q \bar{q}$ as usual, see, for example $[54,55,56]$. Alternatively, it can be given a substructure of diquarks $(q q-\bar{q} \bar{q})$ according to the scheme by Jaffe [57] within the MIT bag model where the nonet is constructed as the direct product of the anti-triplet of diquarks ( $u d, u s, d s)$ and the triplet of antidiquarks $(\bar{u} \bar{d}, \bar{u} \bar{s}, \bar{d} \bar{s})$. This construction explains naturally the ordering in mass of the states where $a_{0}, f_{0}$ have two, $\kappa$ has one and $\sigma$ has no $s(\bar{s})$ quark constituent

$$
\begin{gathered}
a^{+}=[s u][\bar{s} \bar{d}], \quad f_{0} / a^{0}=([s u][\bar{s} \bar{u}] \pm[s d][\bar{s} \bar{d}]) / \sqrt{2}, \quad a^{-}=[s d][\bar{s} \bar{u}] \\
\kappa^{+}=[s u][\bar{u} \bar{d}], \quad \kappa^{0}=[s d][\bar{u} \bar{d}], \quad \bar{\kappa}^{0}=[u d][\bar{s} \bar{d}], \quad \kappa^{-}=[u d][\bar{s} \bar{u}] \\
\sigma=[u d][\bar{u} \bar{d}]
\end{gathered}
$$

Various applications of the 4-quark model for the light scalars have been considered for decay and production processes, in particular with photons [58, 59]. A picture of these scalar mesons as a mixture of tetra-quark states (dominating in the light mesons) and heavy $(q \bar{q})$ states (dominating the heavier mesons) has been proposed in [60] based on an instanton induced effective Lagrangian theory. The mixing between the mass eigenstates $f_{0}$ and $\sigma$ and the ideally mixed states in (27) is typically assumed small to be consistent with the near mass degeneracy of $f_{0}$ and $a_{0}$. For example, the corresponding mixing angle is $|\omega|<5^{\circ}$ in [60].

The light scalar mesons can be established by the motion of phase shifts through $90^{\circ}$ in the appropriate two-body processes, except for the $\kappa$ with a phase in $K \pi$-scattering staying below $40^{\circ}$ (see section 5.2).

\subsection{Route 2: light glueball around $1 \mathrm{GeV}$}

The QCD results discussed above do not allow a definitive conclusion about the mass and properties of the lightest scalar glueball. A light glueball near $1 \mathrm{GeV}$ is obtained from QCD sum rules; based on low energy theorems also a large width is suggested which points to $f_{0}(500)$, sometimes also referred to as $f_{0}(500-1000)$ according to the heavier Breit-Wigner mass of $\sim 1000 \mathrm{MeV}$ (see below and section 5.1.1). A light glueball near $1000 \mathrm{MeV}$ is also expected from certain lattice calculations [21] (see section 5.1.1), both states $f_{0}(500-1000)$ or $f_{0}(980)$ are possible candidates.

In view of the theoretical ambiguities at the end of the 90's, a largely phenomenological approach to light scalars including a glueball has been pursued by 
Minkowski and Ochs [61] without reference to the above specific QCD results. General properties, especially mixing patterns are studied basing on an effective action for sigma variables [62]. The lightest $q \bar{q}$ nonet is constructed from the list of well established resonant states, see table 6 . In particular, the existence of $f_{0}(1370)$ as an extra state has not been accepted: the peaks appearing in the $\pi \pi$ mass spectrum have been interpreted as caused by a broad object centered around $1 \mathrm{GeV}$ interfering destructively with the narrow resonances $f_{0}(980)$ and $f_{0}(1500)$ ("red dragon" phenomenon). This broad object has been related to $f_{0}(500)$ : after separation of $f_{0}(980)$ the $\pi \pi$ elastic phase shifts pass $90^{\circ}$ near $1000 \mathrm{MeV}$ and can be represented locally by a "Breit-Wigner" resonance. A typical fit for " $f_{0}(1000)$ " yields (see also [63])

$$
M_{B W} \approx 1000 \mathrm{MeV}, \quad \Gamma_{B W} \approx 700 \mathrm{MeV} .
$$

The determination of the amplitude pole from the phase shifts is non-trivial and we come back to this question in section 5.1.1.

The lightest $q \bar{q}$ nonet in that scheme includes the isoscalars $f_{0}(980)$ and $f_{0}(1500)$ with a mixing similar to $\eta^{\prime}-\eta$. This has been motivated by the observed decay rates $J / \psi \rightarrow \omega, \phi+X$ which project out the strange and non-strange quark components of $X \|$ and are found comparable for $X=\eta^{\prime}$ and $X=f_{0}(980)$. According to PDG [4] (in units of $\left.10^{-4}\right)$ :

$$
\begin{array}{ll}
B\left(J / \psi \rightarrow \omega \eta^{\prime}\right)=1.82 \pm 0.21 ; & B\left(J / \psi \rightarrow \phi \eta^{\prime}\right)=4.0 \pm 0.7 \\
B\left(J / \psi \rightarrow \omega f_{0}(980)\right)=1.4 \pm 0.5 ; & B\left(J / \psi \rightarrow \phi f_{0}(980)\right)=3.2 \pm 0.9
\end{array}
$$

We write the flavour mixing of these scalar mesons as

$$
\begin{aligned}
& \left|f_{0}(980)\right\rangle=\sin \phi_{s c}|n \bar{n}\rangle+\cos \phi_{s c}|s \bar{s}\rangle, \\
& \left|f_{0}(1500)\right\rangle=\cos \phi_{s c}|n \bar{n}\rangle-\sin \phi_{s c}|s \bar{s}\rangle,
\end{aligned}
$$

or inversely

$$
\begin{aligned}
& |n \bar{n}\rangle=\sin \phi_{s c}\left|f_{0}(980)\right\rangle+\cos \phi_{s c}\left|f_{0}(1500)\right\rangle \\
& |s \bar{s}\rangle=\cos \phi_{s c}\left|f_{0}(980)\right\rangle-\sin \phi_{s c}\left|f_{0}(1500)\right\rangle .
\end{aligned}
$$

\|I It is assumed that the "singly disconnected diagram", where the $q \bar{q}$ is produced in a single loop through one intermediate gluonic exchange, dominates over the "doubly disconnected diagrams" [40].

Table 6. Spectroscopy with glueballs, route 2: light glueball around $1 \mathrm{GeV}$.

\begin{tabular}{cccc}
\hline$I=0$ & $I=\frac{1}{2}$ & $I=1$ \\
\hline \multicolumn{3}{c}{$K^{*}(1950)$} & \\
\hline$f_{0}(1710)$ & & \\
\hline$f_{0}(1500)$ & $K^{*}(1430)$ & & $q \bar{q}$ nonet: \\
$\left(f_{0}(1370) ?\right)$ & & $f_{0}(980)-f_{0}(1500)$ mixed \\
$f_{0}(980)$ & & $a_{0}(980)$ & like $\eta-\eta^{\prime}$ \\
\hline & $\left(K^{*}(900) / \kappa ?\right)$ & light glueball $\quad \mathrm{m} \sim 1 \mathrm{GeV}$ \\
$f_{0}(500) / \sigma$ & & &
\end{tabular}


For estimates, the flavour composition $|u \bar{u}, d \bar{d}, s \bar{s}\rangle$ has been approximated by

$$
\eta^{\prime}, f_{0}(980) \leftrightarrow|1,1,2\rangle / \sqrt{6} ; \quad \eta, f_{0}(1500) \leftrightarrow|1,1,-1\rangle / \sqrt{3} .
$$

The octet formed from $a_{0}(980), K_{0}^{*}(1430)$ and $f_{0}^{\prime} \equiv f_{0}(1500)$ is found to follow the Gell-Mann-Okubo mass relation (using masses 984.7, 1412 and $1507 \mathrm{MeV}$ )

$$
\begin{aligned}
& m^{2}\left(f_{0}^{\prime}\right)=m^{2}\left(a_{0}\right)+\frac{4}{3}\left(m^{2}\left(K_{0}^{*}\right)-m^{2}\left(a_{0}\right)\right) \\
& 2.271=0.970+1.365=2.335 \quad\left[\mathrm{GeV}^{2}\right]
\end{aligned}
$$

within a few percent.

The correspondence $\eta^{\prime} \leftrightarrow f_{0}(980)$ and $\eta \leftrightarrow f_{0}(1500)$ is also characteristic of the "Bonn quark model" [64, 65] and the model of Nambu-Jona-Lasinio type [66] which include instanton interactions with axial $U(1)$ symmetry-breaking [67]; these models explain the reversed mass differences between the octet and singlet states in the scalar and pseudoscalar nonets.

The extra state $f_{0}(500-1000)$ is then supernumerous and is taken as glueball. This identification is supported at first view by the observation of this state in all "gluon rich" processes (i)-(v), with the possible exception of $J / \psi \rightarrow \gamma \pi^{0} \pi^{0}$ which is difficult to select from background [68]. 9 Other results within this scheme are presented in [61] and on the appearance in $D, D_{s}$ and $B$ decays in [70] and [48]. The $\kappa$ meson is not required in this scheme. Further discussion follows in the subsequent sections on $f_{0}^{\prime} s$.

\subsection{Route 3: two supernumerous states, broad glueball $f_{0}(1200-1600)$}

A global description of processes with mesonic final states $\pi \pi, K \bar{K}, \eta \eta, \eta \eta^{\prime}, 4 \pi$ with $J^{P C}=0^{++}$in the mass range $280-1900 \mathrm{MeV}$ in terms of a $K$ matrix and also using a dispersion relation method has been presented by Anisovich et al. [71] (earlier work in $[36,37])$. Data from $\bar{p} p, \bar{p} n$ annihilation into three mesons and meson pair production in $\pi p$ collisions have been included in a global fit. The appropriate mixing angle $\phi$ is determined for each resonance from their decays (following table 3) where the glueball decay is "flavour blind" with a modification from strange quark suppression. The two isoscalar states in a nonet are orthogonal in flavour space and the mixing angles of the respective states should fulfill

$$
\phi^{(I)}-\phi^{(I I)}= \pm 90^{\circ} .
$$

The results of the fits suggested the classification of the isoscalar states as in table 7 with two $q \bar{q}$ nonets and one broad state $f_{0}(1200-1600)$ (width $\left.\Gamma=1100 \pm 140\right)$ identified as glueball. Alternatively, one could exchange the roles of $f_{0}(1300)$ and $f_{0}(1200-1600)$ which are both near flavour singlet. The $\sigma$ meson is another extra state and it is related to a confinement singularity of the $q \bar{q}$ potential.

I Preliminary data from BES-III suggest a contribution from $f_{0}(500)$ centered at low masses around $500 \mathrm{MeV}[69]$. 
Table 7. Spectroscopy with glueballs route 3: two supernumerous states.

\begin{tabular}{lll}
\hline$\left[f_{0}(980), f_{0}(1300)\right]$ & {$\left[f_{0}(1500), f_{0}(1750)\right]$} & $q \bar{q}$ nonets \\
$f_{0}(1200-1600)$ & $f_{0}(500) / \sigma$ & glueball, extra state \\
\hline
\end{tabular}

There is some similarity with route 2 in that the broad object is of gluonic origin. The broad "background" under the narrow resonances in elastic $\pi \pi$ scattering is represented by two poles in route 3 and by one pole in route 2 corresponding to the appearance of only one loop of the amplitude in the complex plane after the narrow states are removed. Furthermore, both schemes work without $K^{* 0}(900) / \kappa$. On the other hand, the fits of route 3 include the state $f_{0}(1300)$ which is not included in route 2 .

\section{Properties of $f_{0}(500) / \sigma$ - comparison with $K_{0}^{*}(800) / \kappa$}

\subsection{Results on $f_{0}(500) / \sigma$}

5.1.1. Mass and width. There are different definitions of the mass of an unstable particle. If $P(s) \simeq 1 / D(s)$ is its propagator a conventional definition is the "on shell" mass with $\operatorname{Re} D\left(s_{0}\right)=0$ in analogy to the treatment of stable particles. A more fundamental definition is the "pole mass" with $D(\bar{s})=0$. As this definition is a basic property of the $S$-matrix one expects in general better properties, for example, gauge invariance in gauge theories; both quantities can be related (see, for example [72]).

In the "Breit Wigner" approximation for masses around the resonance peak the scattering amplitude is written in terms of resonance mass $m_{0}=\sqrt{s_{0}}$ and width $\Gamma$ as

$$
T \sim \frac{1}{s_{0}-s-i m_{0} \Gamma(s)}, \quad \cot \delta=\frac{s_{0}-s}{m_{0} \Gamma(s)}
$$

and the "on shell" or "Breit Wigner" mass is defined by $\operatorname{Re} T^{-1}=0$ or $s=s_{0}$, i.e. at this mass the amplitude becomes purely imaginary and the phase passes $90^{\circ}$; the width is obtained from $\frac{d \delta}{d \sqrt{s}}=\frac{2}{\Gamma}$ at $s=s_{0}$. In this way the Breit-Wigner mass and width are experimentally observable quantities. For $f_{0}(500-1000) / \sigma$ one finds typically $(28)$. These Breit-Wigner quantities are also relevant in the applications of the QCD sum rules, where they enter in the narrow width approximation to the dispersion relations as in (7). The complex pole mass $\sqrt{\bar{s}}=\bar{m}-i \frac{\bar{\Gamma}}{2}$ is not directly accessible from experiment but an analytic extrapolation of the amplitude away from the region of experimental measurements is required.

A description of the observed $\pi \pi$ phase shifts from threshold to $\sim 1400 \mathrm{MeV}$ has been achieved using a "minimal meromorphic parameterization" [73] which is defined for a resonance from its complex pole position and a width $\Gamma(s) \propto q$. Three resonances have been included and a background taking care of the Adler zero. Then the pole of the broad resonance is close to the Breit Wigner result (28).

Another procedure to determine the pole mass is based on the application of partial wave dispersion relations ("Roy equations" [74]) which follow from first principles of 
$S$ matrix theory such as analyticity, crossing symmetry and unitarity, together with experimental information on $\pi \pi$ scattering. The pole of the amplitude at complex energy $\sqrt{\bar{s}}=\bar{m}-i \bar{\Gamma} / 2$ has been found in that way as [75]

$$
\bar{m}_{\sigma}=441_{-8}^{+16} \mathrm{MeV}, \quad \bar{\Gamma}_{\sigma}=544_{-25}^{+18} .
$$

Other applications of dispersion relations for an extended range of energy $(\sqrt{s} \lesssim 1400$ $\mathrm{MeV}$ ) lead to similar results with $\bar{m}_{\sigma}=457_{-13}^{+14}, \bar{\Gamma}_{\sigma}=558_{-14}^{+22} \mathrm{MeV}[76]$ and $\bar{m}_{\sigma}=$ $442_{-8}^{+5}, \bar{\Gamma}_{\sigma}=548_{-10}^{+12} \mathrm{MeV}$ [77] (For further discussion, see [78]).

The large difference between the pole mass $\bar{m}_{\sigma}$ and the "Breit-Wigner mass" or "on shell mass" $M_{\sigma}^{B W}$ where the phase shift passes $90^{\circ}$ can be understood in simple models such as the one by Mennessier [79] which has been slightly modified for recent applications [80, 81]. The analytical and unitary amplitudes for $\pi \pi \rightarrow \pi \pi, K \bar{K}$ are derived from a $K$ matrix parameterization with $\sigma$ and $f_{0}(980)$ resonances where the real part of the amplitudes is derived from dispersion relations. One finds for the pole and "on-shell" mass

$$
\bar{m}_{\sigma} \simeq 422 \mathrm{MeV}, \bar{\Gamma}_{\sigma} \simeq 580 \mathrm{MeV} ; \quad m_{\sigma}^{B W} \simeq 900 \mathrm{MeV}, \Gamma_{\sigma \rightarrow \pi \pi}^{B W} \simeq 1000 \mathrm{MeV},
$$

so $\bar{m}_{\sigma}$ and $\bar{\Gamma}_{\sigma}$ are found near (40). The difference between pole and "on shell" mass is related in this model to the analytic properties of the denominator $D(s)$ of the $\sigma$ propagator. ${ }^{+}$

5.1.2. Phenomenological appearance of $f_{0}(500) / \sigma$. In $\pi \pi$ elastic scattering the $f_{0}(500) / \sigma$ contribution corresponds to a broad resonance with central mass near 1000 $\mathrm{MeV}$ where the phase passes through $90^{\circ}$. In decays of mesons of low mass, such as $f_{0}(1500) \rightarrow \sigma \sigma$, only the low energy part of $\sigma$ below $1 \mathrm{GeV}$ contributes because of phase space restrictions. In decays of $B$ mesons with heavy $b$ quark the broad background appears again which can be related to $f_{0}(500) / \sigma$, for example in $B \rightarrow K \pi \pi, B \rightarrow K \bar{K} K$ (Belle [82]) and $B_{s}^{0} \rightarrow J / \psi \pi \pi$ (LHCb [83]). The Belle data have been parameterized in [48] by a broad resonance with moving phase as in elastic scattering. There are other decays, like $J / \psi \rightarrow \omega \pi \pi[84,85]$ or $D \rightarrow 3 \pi[86]$ where a low mass peak around $500 \mathrm{MeV}$ appears. The appearance of this peak in processes of relatively low energy and absence at higher energies may be related to a special dynamics not directly related to the $\sigma$ pole, for example to subthreshold $\rho$ exchange with primary $J / \psi \rightarrow \rho \pi$ and secondary $\rho \rightarrow \omega \pi$ transition [87]. In decays like $\chi_{c} \rightarrow \sigma \sigma$ it is not obvious from the beginning, what mass spectrum the $\sigma$ decays will acquire. Some clue may come from a comparison of $\chi_{c 0}$ and $\chi_{c 2}$ with the very different phase space factors. Independently of the position of the mass peak, the final state $\pi \pi$ phase should follow the phase of elastic scattering if re-interactions are neglected. This was observed for $J / \psi \rightarrow \omega \pi \pi$ where the $S-D$ wave interference behaves smoothly over the peak region around $500 \mathrm{MeV}$ as reported in $[88]$.

\footnotetext{
+ Earlier phenomenological pole determinations by BES [85] using a variety of parameterizations gave
} the pole position $\sqrt{\bar{s}}=(541 \pm 39)-i(252 \pm 42) \mathrm{MeV}$. 
Table 8. Ratio of couplings $R_{\sigma K \pi}=g_{K \bar{K}}^{2} / g_{\pi \pi}^{2}$ and $R_{\sigma \eta \pi}=g_{\eta \eta}^{2} / g_{\pi \pi}^{2}$ for $f_{0}(500) / \sigma$.

\begin{tabular}{lllll}
\hline processes & $R_{\sigma K \pi}$ & $R_{\sigma \eta \pi}$ & model & reference mass \\
\hline$\pi \pi \rightarrow \pi \pi / K \bar{K}$ & $0.36_{-0.20}^{+0.27}$ & & K matrix [89] & $\sigma$ pole $/ m_{B W}$ \\
$\pi \pi \rightarrow \pi \pi / K \bar{K} / 4 \pi$ & $0.56_{-0.27}^{+0.36}$ & & S matrix [89] & $\sigma$ pole \\
$\pi \pi \rightarrow \pi \pi / K \bar{K} / \eta \eta$ & $0.6_{-0.2}^{+0.1}$ & $0.2 \pm 0.04$ & resonance model [90] & $m_{B W} \gtrsim 1 \mathrm{GeV}$ \\
\hline
\end{tabular}

5.1.3. Couplings of $f_{0}(500) / \sigma$ to $\pi \pi, K \bar{K}$ and $\eta \eta$. The intrinsic structure of $f_{0}(500)$ can be revealed by the decay branching ratios. These can be extracted from a model amplitude which fits data from both channels $\pi \pi$ and $K \bar{K}$. The decay ratio of couplings $r_{\sigma \pi K}=\frac{\left|g_{\sigma K^{+} K^{-}}\right|}{\left|g_{\sigma \pi^{+} \pi^{-}}\right|}$has been derived from an extrapolation to the pole of an analytical amplitude or "on shell" along the real axis near $m_{B W} \sim 1 \mathrm{GeV}$. A result of the first kind is obtained by Kaminski et al. [89] based on a coupled channel analysis as described in the previous section 5.1.1 using the analytical $K$-matrix model [79, 80]. They find $r_{\sigma K \pi}=0.52 \pm 0.17$, i.e. a significant strange quark component in the $\sigma$. In table 8 we summarize the results on the ratios $R_{\sigma K \pi}=\frac{g_{\sigma \rightarrow K \bar{K}}^{2}}{g_{\sigma \rightarrow \pi \pi}^{2}}=\frac{4}{3} r_{\sigma K \pi}^{2}$ and $R_{\sigma \eta \pi}$ which are derived from fits to both the elastic and inelastic $\pi \pi$ scattering. In an approach based on dispersion relations and a 3-coupled channel unitary model a similar result has been obtained by the same group $r_{\sigma K \pi}=0.65 \pm 0.18$. A phenomenological description of $\pi \pi$, $K \bar{K}$ and $\eta \eta$ channels extending up to $1.6-2.0 \mathrm{GeV}$ based on extended inelastic Breit Wigner formulae for $f_{0}(500) / \sigma$ and inclusion of other resonances has been achieved by Bugg [90]. Near threshold the process $\pi \pi \rightarrow K \bar{K}$ is dominated by $f_{0}(980)$ but a sizable $f_{0}(500) / \sigma$ component with $R_{\sigma K \pi}=0.6_{-0.2}^{+0.1}$ is necessary for a good fit. We note however, that this quantity may be subject to additional systematic uncertainties because of conflicting experimental phases just above the $K \bar{K}$ threshold: the fit by Bugg follows the phases by Etkin et al. [91] which start rising near $\varphi_{t h r} \sim 170^{\circ}$ while the phases by Cohen et al. [92], preferred in figure 12 below, start decreasing from $\varphi_{t h r} \sim 220^{\circ} *^{*}$

The results for $R_{\sigma K \pi}$ in table 8 show consistency between both methods. For the K-matrix model it has been found [89] that $r_{\sigma K \pi}$ evaluated for the pole mass and for the on-shell (BW) mass coincide. The ratios in table 8 are all derived from the same data and we estimate

$$
R_{\sigma K \pi}=0.5 \pm 0.2 \text {. }
$$

There is also a small but significant decay into $\eta \eta$ with $R_{\sigma \eta \pi}=0.20 \pm 0.04$.

All these results indicate that $f_{0}(500) / \sigma$ is not a pure non-strange object, but couples to a sizable $s \bar{s}$ (or gluonic) component. A pure flavour singlet or glueball would correspond to $r_{\sigma K \pi}=1$ or $R_{\sigma K \pi}=4 / 3$ which is above the results in table 8 . While some caution on these numbers is still appropriate because of possible systematic errors there is also the possibility of a mixing with $f_{0}(980)$ as discussed in [32]. According to these model results, a 4-quark model in its simplest form with a non-strange $\sigma$ [57] or

* Bugg also obtained a consistent result $R_{\sigma K \pi}=0.6 \pm 0.1$ from data on the radiative decay $\phi(1020) \rightarrow \gamma \pi^{0} \pi^{0}$ with the contribution of the virtual process $\pi \pi \rightarrow K \bar{K}$ within the $K \bar{K}$ loop model. 
with a small mixing $r_{\sigma \pi K}<0.1$ as in [93] is not supported.

5.1.4. Two-photon coupling of $f_{0}(500) / \sigma$. Following the general idea that glueballs couple weakly to photons one may consider the two-photon decay of the tentative glueball as a crucial measurement. The recent results from Belle [94, 95] on the reactions $\gamma \gamma \rightarrow \pi^{0} \pi^{0}, \pi^{+} \pi^{-}$have renewed the interest in this problem. The two-photon width has been extracted by Pennington [96] as $\Gamma(\sigma \rightarrow \gamma \gamma)=(4.1 \pm 0.3) \mathrm{keV}$. This result has been obtained from the application of partial wave dispersion relations and using the position of the $\sigma$ pole (40) from [75]. A more recent evaluation and the mean of various other determinations [77] is a bit lower

$$
\Gamma(\sigma \rightarrow \gamma \gamma)=\left(2.08 \pm 0.20_{-0.04}^{+0.07}\right) \mathrm{keV}
$$

A number of this order is in the range of expectations for $q \bar{q}$ states but gluonic states require generally smaller decay widths, for example $\Gamma(\sigma \rightarrow \gamma \gamma) \approx 0.2-0.3 \mathrm{keV}$ [97], an order of magnitude smaller than what is found in (43). Therefore the glueball assignment has been questioned in [96].

A possible solution of this problem has been suggested in [80] within the analytic $K$ matrix model mentioned before [79]. Given a set of coupled multi-channel strong processes, the amplitudes for the related electromagnetic processes are largely determined by unitarity and constraints from dispersion relations, although with some ambiguities from subtraction terms [98]. Along this general scheme, the calculation of the electromagnetic processes $\gamma \gamma \rightarrow \pi \pi, K \bar{K}$, given the strong processes $\pi \pi \rightarrow \pi \pi, K \bar{K}$ has been carried out in [79] based on a $K$ matrix model representing the amplitudes by a set of poles. The subtraction ambiguities in electromagnetic processes correspond to the free "direct couplings" of resonances to photons.

At low energies the process $\gamma \gamma \rightarrow \pi^{+} \pi^{-}$is governed by one-pion exchange with the photons coupling to charged pions and this process dominates by an order of magnitude over $\gamma \gamma \rightarrow \pi^{0} \pi^{0}$ where the initial photons couple to charged pions and subsequently re-scatter: $\gamma \gamma \rightarrow \pi^{+} \pi^{-} \rightarrow \pi^{0} \pi^{0}$. In the resonant scattering $\gamma \gamma \rightarrow R \rightarrow \pi \pi$ there is the possibility of $\pi \pi$ re-scattering and in addition of "direct coupling" $\gamma \gamma \rightarrow R$. At higher energies the re-scattering contribution decreases and the direct processes become dominant. They correspond to interactions where the energetic photons resolve the constituents inside the resonances. An example is the two-photon decay of $f_{2}(1270)$ and other tensor mesons which are well represented by the coupling of photons to the constituent quarks [3].

In [80] the "direct coupling" of $f_{0}(500) / \sigma$ has been determined from the simultaneous fits to the strong $\pi \pi$ and the $\gamma \gamma$ processes below $700 \mathrm{MeV}$. Subsequently, the inelastic channels including $K \bar{K}$ and a second resonance $f_{0}(980)$ has been included $[89,81]$. The following results at the resonance pole for $f_{0}(500) / \sigma$ are obtained

$$
\begin{aligned}
& \Gamma_{\sigma}^{\text {dir }} \rightarrow \gamma \gamma \simeq(0.13 \ldots 0.19) \mathrm{keV}, \\
& \Gamma_{\sigma}^{\mathrm{resc}} \rightarrow \gamma \gamma \simeq(2.7 \ldots 1.48) \mathrm{keV}, \quad \Gamma_{\sigma}^{\text {tot }} \rightarrow \gamma \gamma \simeq(3.9 \ldots 2.67) \mathrm{keV}
\end{aligned}
$$


where the first number comes from [80], the second one from the average of the two numbers in [81]. One can see that with this approach the $\gamma \gamma$ process is dominated by $\pi \pi$ re-scattering. It is the direct coupling component which should be related to the constituents of the resonance and this is an order of magnitude smaller than the total width, quite compatible with gluonic constituents (like $\Gamma \sim 0.2-0.3 \mathrm{keV}$, as in [97]). This result is based on a model consideration, but one may conclude that it is premature to reject the gluonic nature of $\sigma$ based on the $2 \gamma$ decay width.

\subsection{Comparing elastic $\pi \pi$ and $K \pi$ scattering - a problem with the $\kappa$ resonance}

In the nonet classification of the scalars below $1000 \mathrm{MeV}$ (route 1 ) the broad $\kappa$ and $\sigma$ particles appear. While $\sigma$ plays its role in all three classification routes considered the $\kappa$ appears only in route 1 and would be supernumerous otherwise if a real resonance. In this subsection we add a few comments on these broad objects in a comparison.

At first sight there is a similarity in low energy $\pi \pi$ and $K \pi$ scattering and the strong low energy interaction suggests the existence of two broad resonances $\sigma$ and $\kappa$ with a width comparable to mass. The first narrow resonance of low mass in $\pi \pi$ scattering appears with $f_{0}(980)$ and in $K \pi$ scattering with $K_{0}^{*}(1430)$. A closer look at the scattering phase shifts, however, reveals a considerable difference between both scattering channels.

In the elastic scattering region we may parameterize the amplitude for the production of a resonance $R$ above a broad background $B$, following Michael [99], as product of the respective $S$ matrices which satisfies unitarity (also called "Dalitz-Tuan method")

$$
S=S_{R} S_{B}, \quad \text { or } \quad \mathrm{T}=\mathrm{T}_{\mathrm{B}}+\mathrm{e}^{2 \mathrm{i} \delta_{\mathrm{B}}} \mathrm{T}_{\mathrm{R}}
$$

where $S=1+2 i T$ and $T=\sin \delta e^{i \delta}$. This approximation applies below the inelastic thresholds of $\pi \pi \rightarrow K \bar{K}$ at $m_{\pi \pi} \sim 1000 \mathrm{MeV}$ and $K \pi \rightarrow K \eta^{\prime}$ at $m_{K \pi} \sim 1450 \mathrm{MeV}$ respectively (disregarding the negligible $K \eta$ production) and then the overall phase of $S=e^{2 i \delta}$ is $\delta=\delta_{R}+\delta_{B}$. According to [99] (46) is also applicable for inelastic resonances and near elastic backgrounds. If the contribution of the lowest narrow resonances $R$ to the phase $\delta$ are "removed" the remaining background phase $\delta_{B}$ shows quite a different behaviour for the two channels (see figure 5):

$$
\begin{aligned}
& m_{\pi \pi} \approx 1000 \mathrm{MeV}: \delta_{B}=90^{\circ} \\
& m_{K \pi} \approx 1400 \mathrm{MeV}: \delta_{B}<40^{\circ},
\end{aligned}
$$

i.e. in $\pi \pi$ scattering the "background" itself shows a resonant behaviour whereas in $K \pi$ scattering the background phase saturates already below $40^{\circ}$ according to the present experimental status of $K \pi$ scattering which presents itself as follows:

There is still a discussion on the appropriate description of low energy $K \pi$ scattering. Elastic $K \pi$ scattering amplitudes have been reconstructed from processes $K^{ \pm} p \rightarrow K^{ \pm} \pi^{+} n$ and $K^{ \pm} p \rightarrow K^{ \pm} \pi^{-} \Delta^{++}$by isolating the respective One-Pion-Exchange contribution [106, 105]. Following the LASS Collaboration [105] the S-wave contribution 

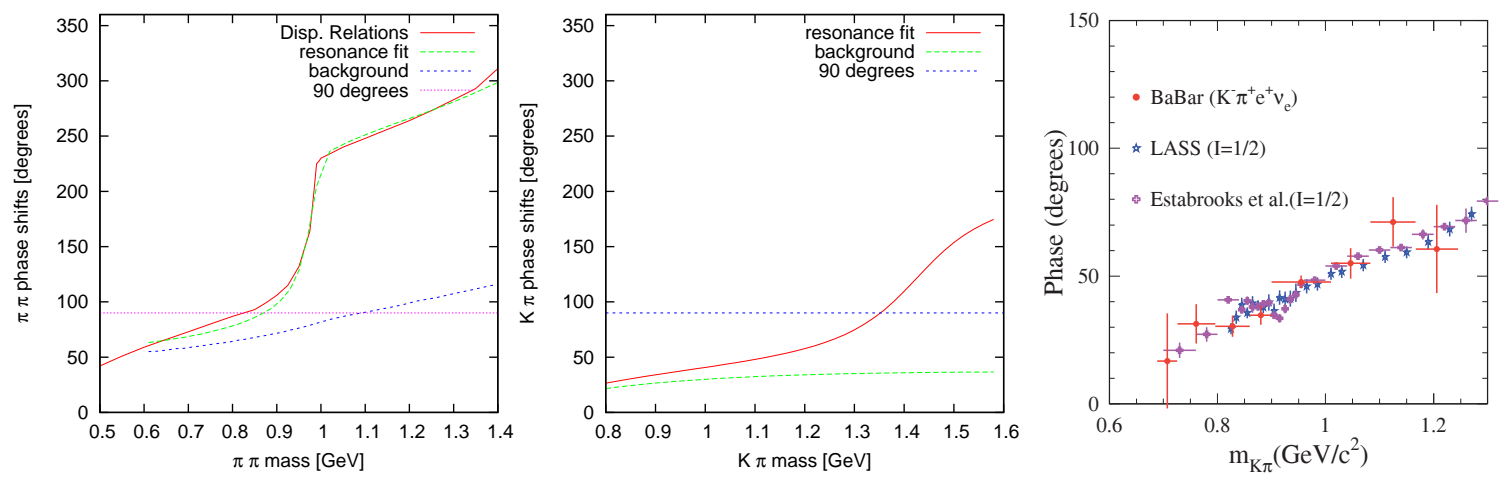

Figure 5. Phase shifts of elastic scattering, Left: $\pi \pi$ phase shifts from Dispersion Relations [100] and from resonance fit to CM-I data [101] $\left(f_{0}(980)+\right.$ background [102]); Middle: $K \pi$ phase shifts $\left(K_{0}^{*}(1430)+\right.$ background [103, 104]); Right: $K \pi$ phase shifts from BABAR [103] $\left(D^{+} \rightarrow K^{-} \pi^{+} e^{+} \nu_{e}\right)$, LASS [105] $\left(K^{ \pm} p \rightarrow K^{ \pm} \pi^{+} n\right)$ and Estabrooks et al. [106] $\left(K^{ \pm} p \rightarrow K^{ \pm} \pi^{-} \Delta^{++}\right)$, figure from [103]. The $K \pi$ background phase stays below $40^{\circ}$.

with $I=\frac{1}{2}$ can be represented by the superposition as in (46) of the $K_{0}^{*}(1430)$ BreitWigner amplitude and a background which is parameterized by an effective range formula (cms momentum $k$ )

$$
\cot \left(\delta_{\mathrm{BG}}\right)=\frac{1}{a k^{2}}+\frac{b k^{2}}{2}
$$

a recent reanalysis has been presented by the BABAR Collaboration $[103,104]$ which gave the numbers $a=1.95 \pm 0.09 \mathrm{GeV}^{-1}$ and $b=1.76 \pm 0.36 \mathrm{GeV}^{-1}$ for the $I=\frac{1}{2}$ S-wave.

A model independent determination of the $K \pi$ phases in the elastic region is possible from the decays of $D \rightarrow K \pi e \nu_{e}$ and $\tau \rightarrow K \pi \nu_{\tau}$ as in these decays, according to the Watson theorem [107], the $K \pi$ production phase agrees (modulo $\pi$ ) with the elastic $K \pi$ scattering phases.

A measurement of all the decay distributions in $D \rightarrow K \pi e \nu_{e}$ by the BABAR Collaboration [103] has determined the $I=\frac{1}{2} K \pi S$-wave phase shifts in the range $700-1400 \mathrm{MeV}$. The results agree nicely with the earlier results on $K \pi$ phases from the production off the proton target and confirm the description of the $S$-wave amplitude in terms of the $K_{0}^{*}(1430)$ and the effective range background (49) (see figure 5, right panel). The resulting background phase approaches $38^{\circ}$ at around $1400 \mathrm{MeV}$ where it reaches a maximum. $\sharp$

An analysis of $\tau \rightarrow K \pi \nu_{\tau}$ decays has been presented by Belle [109]. The $K \pi$ mass spectrum requires contributions in addition to $K^{*}(892)$ and a successful fit includes an $S$-wave with a superposition of $K_{0}^{*}(800) / \kappa$ and $K_{0}^{*}(1430)$. Also a LASS-type fit with background (49) was possible but found only marginally consistent with previous values \# The existence of an $S$-wave with a phase variation consistent with (49) has been noted before [108] in a limited mass range around $K^{*}(892)$ with lower statistics. 
for the parameters $a$ and $b$. However, the measurement of further decay observables is required for an independent determination of the $K \pi$ phase shifts and a test of the Watson theorem, to compare with the results from $D \rightarrow K \pi e \nu_{e}$. First results have been presented by BABAR with similar fits [110]. At present we stay with the results presented in figure 5.

The situation with a low mass resonance $K_{0}^{*}(800) / \kappa$ is discussed by the PDG [78]. The existence of this state close to the $K \pi$ threshold and with a large width around $500 \mathrm{MeV}$ is considered difficult to establish. A positive result is obtained recently in an approach based on a dispersive representation of the $K \pi$ scattering amplitude [111]. After continuation into the complex energy plane a pole has been identified at

$$
\bar{m}_{\kappa}=658 \pm 13 \mathrm{MeV}, \quad \Gamma_{\kappa}=557 \pm 24 \mathrm{MeV},
$$

just above $K \pi$ threshold at around $640 \mathrm{MeV}$.

In summary, descriptions of the scalar $K \pi$ amplitude with a $\kappa$ pole built in are possible but the apparent slow phase motion of the corresponding amplitude which saturates below $40^{\circ}$ is untypical for a resonance. In this way a characteristic condition for the existence of a resonance - the phase shifts are passing through $90^{\circ}$ - is not fulfilled. One could think of a repulsive background under the $\kappa$ with destructive interference [112], but then the background phase ought to be larger than the resonance phase $\left(\left|\delta_{B}\right|>\left|\delta_{R}\right|\right)$. Alternatively, an enhanced but not necessarily resonant low energy $K \pi$ interaction could reflect the strong $t$-channel $\pi \pi \rightarrow K \bar{K}$ low energy $(\sigma)$ forces. $\dagger \dagger$

\section{Intrinsic structure of $f_{0}(980)$}

\subsection{Mixing angle from various observables}

We discuss here several observables within the $q \bar{q}$ constituent model and determine the $q \bar{q}$ mixing angle defined as in (31) following earlier studies [61, 70]. Some of these observables have been also considered in [113]; other determinations of the mixing angle, also in the context of a 4-quark picture, have been reported in [114], but no clear picture has emerged. We consider the following ratios of branching ratios:

(i) Decay into $\pi \pi$ and $K \bar{K}$. This decay is often described in terms of a Breit Wigner resonance formula, following Flatté [115], with amplitude

$$
T_{i j}=\frac{g_{i} g_{j} \sqrt{\rho_{i} \rho_{j}}}{s_{0}-s-i\left(\rho_{\pi} g_{\pi}^{2}+\rho_{K} g_{K}^{2}\right)}
$$

where $g_{i}$ denotes the couplings of the resonance to the $\pi \pi$ and $K \bar{K}$ channels and $\rho_{i}=\frac{2 k_{i}}{\sqrt{s}}$ the respective phase space with the momenta $k_{i}$ in the resonance rest frame. The BES Collaboration has measured the decays $J / \psi \rightarrow \phi(1020) \pi^{+} \pi^{-} / \phi(1020) K^{+} K^{-}$and determined $g_{K}^{2} / g_{\pi}^{2}=4.21 \pm 0.33$ (errors added in quadrature) [116].

††This viewpoint has been envisaged in discussions with P. Minkowski. 
Various results have been obtained from data on the process $\pi \pi \rightarrow K \bar{K}$ together with $\pi \pi \rightarrow \pi \pi$ in an application of different coupled channel approaches. The data mainly used in these analyses because of their accuracy are from Cohen et al. on $K^{+} K^{-}$[92] and from Etkin et al. [91] on $K_{1}^{0} K_{1}^{0}$ final states. In table 9 we list some results on $g_{K}^{2} / g_{\pi}^{2}$ which included $K \bar{K}$ data in the fits: from Martin et al. [117] using

Table 9. Couplings $g_{K}^{2} / g_{\pi}^{2}$ in (51) for $f_{0}(980)$ from $\pi \pi \rightarrow K \bar{K}$ and from $J / \psi$ decay.

\begin{tabular}{lllllll}
\hline Martin & Estabrooks & Bugg & Kaminski & Mennessier & mean $(\pi \pi)$ & BES $(J / \psi)$ \\
$4.0 \pm 0.6$ & $3.2 \pm 1.8$ & 2.3 & $3.9 \pm 0.9$ & $9_{-7}^{+11}$ & $3.3 \pm 0.6$ & $4.21 \pm 0.33$ \\
\hline
\end{tabular}

several different models for resonances; from Estabrooks [118] based on a K matrix fit; from Bugg et al. [119] using a global parameterization based on a $\mathrm{K}$ matrix, see also [113] for interpretation; from Kaminski et al. [120] using a unitary model with separable interactions (see also [89]) and the analytic K-matrix model by Mennessier et al. [81]. As the ratio $g_{K}^{2} / g_{\pi}^{2}$ depends essentially on the same $K \bar{K}$ data we average the weighted results as usual but assume a full correlation of the input data and obtain the mean in table 9. Finally, together with the independent BES measurement we find

$$
f_{0}(980): \quad g_{K}^{2} / g_{\pi}^{2}=4.0 \pm 0.3 ; \quad g_{K} / g_{\pi}>0 .
$$

The same sign of the couplings $g_{K}$ and $g_{\pi}$ has been derived from the fits $[117,118]$ to the $K^{+} K^{-}$data by Cohen et al. In these data the relative sign of the $\pi^{+} \pi^{-} \rightarrow K^{+} K^{-}$ and $\pi^{+} \pi^{-} \rightarrow \pi^{+} \pi^{-}$amplitudes has been fixed from the observation that near $1 \mathrm{GeV}$ the $P$ wave in both channels is governed by the tail of the $\rho$ and assuming these amplitudes to be in phase. This observation resolves the previously noted sign ambiguity (for example in $[113,81])$. The result $(52)$ can be expressed in terms of the mixing angle $\phi_{s c}$ defined in (31). Using table 3 with $\phi_{s c}=\alpha+\pi / 2$ according to the definition (15) we find $g_{K}^{2} / g_{\pi}^{2} \equiv \gamma_{K \bar{K}}^{2} / \gamma_{\pi \pi}^{2}$ as

$$
g_{K}^{2} / g_{\pi}^{2}=\left(1+\sqrt{2} \cot \phi_{s c}\right)^{2} / 3
$$

in the symmetry limit $\rho=1$ and yields $\phi_{s c}=29.9^{\circ} \pm 1.3^{\circ}$ (for $g_{K} / g_{\pi}>0$, otherwise $\phi_{s c}=162.4^{\circ} \pm 0.5^{\circ}$ noting that $g_{K^{+} K^{-}} / g_{\pi^{+} \pi^{-}}=\left(1+\sqrt{2} \cot \phi_{s c}\right) / 2$ from table 3$)$.

(ii) Decays $J / \psi \rightarrow f_{0}(980) \phi$ and $f_{0}(980) \omega$. In these decays the $s \bar{s}$ and $n \bar{n}$ quark components of $f_{0}(980)$ are projected out and according to (31) their ratio can be related to the quark mixing angle [61] assuming the dominance of the "singly disconnected diagram" [40]

$$
R_{J / \psi}^{\phi / \omega}=\frac{B\left(J / \psi \rightarrow f_{0}(980) \phi\right)}{B\left(J / \psi \rightarrow f_{0}(980) \omega\right)}: \quad R_{J / \psi}^{\phi / \omega}=\frac{k_{\phi}}{k_{\omega}} \cot ^{2} \phi_{s c} .
$$

with cms decay momenta $k_{i}$ of $\phi$ and $\omega$. Taking these branching ratios from PDG as in (30) we find $\cot ^{2} \phi_{s c}=2.46 \pm 1.12$ or $\phi_{s c}= \pm(33 \pm 7)^{\circ}$. This result is similar to 
the corresponding ratio for $\eta^{\prime}, R_{J / \psi}^{\eta^{\prime}}=2.05 \pm 0.43$ using PDG values and this yields $\phi_{p s}\left(\eta^{\prime}\right)=(35 \pm 3)^{\circ}$ to be compared with the experimental mixing angle $\phi_{p s}=42^{\circ}$ in $(17 a)$. This observation was the original motivation for the mixing ansatz for scalar mesons in [61].

(iii) Two photon decays of $f_{0}(980)$ and $a_{0}(980)$. These decays are sensitive to the charged constituents of these mesons. In an update of [61] we consider the ratio $R_{\gamma \gamma}$ in a $q \bar{q}$ constituent model

$$
R_{\gamma \gamma}^{f_{0} / a_{0}}=\frac{\Gamma_{\gamma \gamma}\left(f_{0}(980)\right)}{\Gamma_{\gamma \gamma}\left(a_{0}(980)\right)} ; \quad \Gamma_{\gamma \gamma} \propto\left(\sum_{q} c_{q} Q_{q}^{2}\right)^{2}
$$

with amplitudes $c_{q}$ for $q=(u, d, s),\left(\sum c_{q}^{2}=1\right)$, for the contributions from $q \bar{q}$ of charge $Q_{q}$ and obtain with mixing (31)

$$
R_{\gamma \gamma}^{f_{0} / a_{0}}=\frac{25}{9}\left(\sin \phi_{s c}+\frac{\sqrt{2}}{5} \cos \phi_{s c}\right)^{2} .
$$

The two photon decays have been studied recently by the Belle experiment for $f_{0}(980)$ $[94,95]$ and $a_{0}(980)$ [121]. We take here $\Gamma_{\gamma \gamma}^{f_{0}}=0.29_{-0.06}^{+0.07} \mathrm{keV}$ for $f_{0}(980)$ from the PDG fit, in case of $a_{0}$ we use their branching ratio $\Gamma_{K \bar{K}}^{a_{0}} / \Gamma_{\eta \pi}^{a_{0}}=0.183 \pm 0.024$ to obtain $B\left(a_{0}(980) \rightarrow \pi \eta\right)=0.85 \pm 0.02$ and then $\Gamma_{\gamma \gamma}^{a_{0}}=0.25_{-0.05}^{+0.09} \mathrm{keV}$; finally, with (56)

$$
R_{\gamma \gamma}^{f_{0} / a_{0}}=1.16 \pm 0.41, \quad \phi_{s c 1}=(23.7 \pm 8.1)^{\circ} ; \quad \phi_{s c 2}=(125.8 \pm 8.1)^{\circ} .
$$

(iv) Charmed meson decays $D, D_{s} \rightarrow f_{0}(980) \pi$. The decays of $D^{+}$and $D_{s}^{+}$into $f_{0} \pi^{+}$and $K_{0}^{*}(1430) \pi^{+}$provide a further possibility to determine the flavour structure of $f_{0}(980)$ and we follow here the investigation in [70]. They consider the two ratios

$$
R_{D 1}=\frac{\Gamma\left(D_{s}^{+} \rightarrow f_{0} \pi^{+}\right)}{\Gamma\left(D^{+} \rightarrow f_{0} \pi^{+}\right)}, \quad R_{D 2}=\frac{\Gamma\left(D_{s}^{+} \rightarrow f_{0} \pi^{+}\right)}{\Gamma\left(D^{+} \rightarrow K_{0}^{*}(1430) \pi^{+}\right)}
$$

Assuming again the flavour composition (31) for $f_{0}(980)$ and the $K_{0}^{*}(1430)$ to belong to the same nonet, we write the amplitudes for the three processes involved as follows

$$
\begin{array}{rlrl}
\text { (a) } D_{s}^{+} \rightarrow f_{0} \pi^{+} & A & =\cos \phi_{s c} V_{u d} V_{c s}^{*} a \\
\text { (b) } D^{+} \rightarrow f_{0} \pi^{+} & B & =\left[\frac{\sin \phi_{s c}}{\sqrt{2}} V_{u d} V_{c d}^{*}(1+\epsilon)+\cos \phi_{s c} V_{u s} V_{c s}^{*} \epsilon\right] a \\
\text { (c) } D^{+} \rightarrow K_{0}^{*}(1430) \pi^{+} C & =V_{u d} V_{c s}^{*}(1+\epsilon) a .
\end{array}
$$

Process $(a)$ is given by the amplitude with Cabibbo-favoured decay $c \rightarrow s W^{+}$, $W^{+} \rightarrow u \bar{d} \rightarrow \pi^{+}$followed by the spectator interaction $s \bar{s} \rightarrow f_{0}$ with strength $a$. In the Cabibbo-suppressed process $(b)$ there is the same direct $\pi$-emission diagram as in (a) but one has to add the "colour suppressed" amplitudes with decay $c \rightarrow d W^{+}$, $W^{+} \rightarrow u \bar{d}, d \bar{d} \rightarrow f_{0}$ and $c \rightarrow s W^{+}, W^{+} \rightarrow u \bar{s}, s \bar{s} \rightarrow f_{0}$ both of relative size $\epsilon$. Finally in $(c)$ there is one direct and one colour suppressed amplitude. In the approximation $V_{u d}=V_{c s}=\cos \vartheta_{c}, V_{u s}=-V_{c d}=\sin \vartheta_{c}$, with Cabibbo angle $\vartheta_{c}$

$$
\begin{aligned}
& R_{D 1}=2 \frac{q_{a}}{q_{b}} \cot ^{2} \vartheta_{c} \cot ^{2} \phi_{s c} \frac{1}{\left|1-\left(\sqrt{2} \cot \phi_{s c}-1\right) \epsilon\right|^{2}} \\
& R_{D 2}=\frac{q_{a}}{q_{c}} \cos ^{2} \phi_{s c} \frac{1}{|1+\epsilon|^{2}}
\end{aligned}
$$


where $q_{i}$ is the phase space for $S$ wave decays with pion momenta in the rest frame of processes $(a-c)$. Using the branching fractions established by the E791 Collaboration [86] the following results have been found [70]

$$
\begin{aligned}
\cot ^{2} \phi_{s c} /\left|1-\left(\sqrt{2} \cot \phi_{s c}-1\right) \epsilon\right|^{2} & =1.26(1.0 \pm 0.4) \\
\cos ^{2} \phi_{s c} /|1+\epsilon|^{2} & =0.52(1.0 \pm 0.3)
\end{aligned}
$$

with the two solutions

$$
\begin{array}{lll}
\cot \phi_{s c 1}=1.11_{-0.20}^{+0.33}, & \phi_{s c 1}=\left(42.0_{-7.3}^{+5.8}\right)^{\circ}, & \epsilon_{1}=(2.85 \pm 5.35) 10^{-2}(66) \\
\tan \phi_{s c 2}=-0.34_{-0.37}^{+0.46}, & \left.\phi_{s c 2}=161.2_{-16.5}^{+25.7}\right)^{\circ}, & \epsilon_{2}=0.31_{-0.13}^{+0.004}
\end{array}
$$

It is satisfactory that the colour suppressed amplitudes are indeed found small.

Table 10. Quark structure of $f_{0}(980)$ from four observables, mixing angle as in (31).

\begin{tabular}{lllll}
\hline observable & exp. result & sol. $\phi_{s c 1}$ & sol. $\phi_{s c 2}$ & $\eta^{\prime}$-like as in (35) \\
\hline$g_{K}^{2} / g_{\pi}^{2}$ & $4.0 \pm 0.3$ & $(29.9 \pm 1.3)^{\circ}$ & {$\left[(162.4 \pm 0.5)^{\circ}\right]$} & 3 \\
& $g_{K} / g_{\pi}>0$ & $0^{\circ}<\phi_{s c}<90^{\circ}$ & & \\
$R_{J / \psi}^{\phi / \omega}$ & $2.29 \pm 1.04$ & $(33 \pm 7)^{\circ}$ & $(147 \pm 7)^{\circ}$ & 2 \\
$R_{\gamma \gamma}^{f_{0} / a_{0}}$ & $1.16 \pm 0.41$ & $(23.7 \pm 8.1)^{\circ}$ & $(125.8 \pm 8.1)^{\circ}$ & $\frac{49}{27}=1.815$ \\
$R_{D 1}, R_{D 2}$ & $(64),(65)$ & $\left(42.0_{-7.3}^{+5.8}\right)^{\circ}$ & $\left.161.2_{-16.5}^{+25.7}\right)^{\circ}$ & \\
\hline
\end{tabular}

\subsection{Final result on mixing angle}

Our results are summarized in table 10. There is a good agreement between the four determinations for mixing angle solutions with $\phi_{s c 1}<90^{\circ}$, contrary to $\phi_{s c 2}>90^{\circ}$. This can be taken as an independent confirmation of the relative sign of $g_{K} / g_{\pi}$ in (52). Finally, we obtain as average $\phi_{s c}=(30.2 \pm 1.2)^{\circ}$ which is dominated by the observable $g_{K}^{2} / g_{\pi}^{2}$. In view of systematic uncertainties, such as strange quark suppression effects, we estimate the total error to be around $10 \%$, therefore finally

$$
f_{0}(980): \quad \phi_{s c}=(30 \pm 3)^{\circ} .
$$

The mixing angle for $f_{0}(980)$ is then near the mixing angle $(17 b)$ for $\eta^{\prime}$, i.e. $\phi_{s c} \approx$ $\phi_{p s}=42^{\circ}$ as in route 2 , or approximately with $\phi_{s c}=\arccos \sqrt{2 / 3}=35.3^{\circ}$ for $\left|f_{0}(980)\right\rangle \rightarrow(1,1,2) / \sqrt{6}$ as in $(35)$ (observables in table 10 " $\eta^{\prime}$-like") and so $f_{0}(980)$ is close to a flavour singlet state; octet would have $\phi_{s c}>90^{\circ}$. One of the two solutions found in [113], $23^{\circ} \leq \phi_{s c} \leq 37^{\circ}$, is consistent with our result (68).

\subsection{Possible gluonic contribution}

While the data are nicely consistent with $f_{0}(980)$ being a pure quark state, we may estimate a possible gluonic contribution as follows. The fit result (68) which is largely dominated by the result on $g_{K}^{2} / g_{\pi}^{2}$ corresponds to a value $R_{\gamma \gamma}=1.53$ according to (56), 
so the actually measured value in (57) is about $75 \%$ of the nominal value. Taking into account a gluonic component in an extension of (31) by

$$
\left|f_{0}(980)\right\rangle=\cos \beta\left(\sin \phi_{s c}|n \bar{n}\rangle+\cos \phi_{s c}|s \bar{s}\rangle\right)+\sin \beta|g g\rangle
$$

we can write a properly extended equation for $\Gamma_{\gamma \gamma}$ in (55) where the additional gluonic term is of $O\left(\alpha_{s}^{2}\right)$. Neglecting this term here we obtain with the above result

$$
R_{\gamma \gamma} \approx R_{\gamma \gamma}^{q \bar{q}} \cos ^{2} \beta ; \quad \cos ^{2} \beta \approx 0.75 \pm 0.25
$$

i.e. the fraction of a possible gluonic component is about $\frac{1}{4} \pm \frac{1}{4}$. Note that the addition of a gluonic component would leave $R_{J / \psi}^{f_{0}(980)}$ unchanged for fixed $q \bar{q}$ fractions but would modify the other two observables considered.

Although with a considerable uncertainty, this result suggests that more precise data on the above observables could further strengthen the picture and provide better estimates on the amount of glue inside this meson. Recent measurements of decays $D_{s} \rightarrow 3 \pi$ and $D_{s} \rightarrow K \bar{K} \pi[122,123,124]$ have shown the potential of independent determinations of $g_{K}^{2} / g_{\pi}^{2}$. In particular, BaBar has compared the $\pi \pi$ and $K \bar{K}$ mass spectra just around $1 \mathrm{GeV}$ and found good agreement in their shape [123] up to about 1.1 GeV which should directly determine this observable.

More precise results on $R_{J / \psi}^{f_{0}(980)}$ and $\Gamma_{\gamma \gamma}^{f_{0}(980)}$ could improve our knowledge of the flavour structure of $f_{0}(980)$ and are accessible by ongoing or new experiments. A word of caution on the interpretation of the $\gamma \gamma$ results is in order. The model calculations suggested a considerable contribution from hadronic re-scattering in the $\sigma \rightarrow \gamma \gamma$ decay (section 5.1.4). On the other hand, the corresponding calculations for $f_{2}(1270)$ have shown the dominance of the "direct" contribution as expected [81]. Results on $f_{0}(980)$ in between the extremes indicate re-scattering contributions but no strong difference between direct and PDG values for $f_{0}(980) \rightarrow \gamma \gamma$; a strong model dependence on the contributing exchanges can be observed [81]. Further studies, for example with dispersive representations, could strengthen the conclusions. In this presentation we assume the dominance of the direct contribution in (56) as for $f_{2}(1270)$.

\section{Experimental evidence for $f_{0}(1370)$ and $f_{0}(1500)$ - a reassessment}

A crucial precondition of the mixing scenario in route 1 with a supernumerous state in the mass region around $1600 \mathrm{MeV}$ is the existence of $f_{0}(1370)$. This section is devoted to a critical reassessment of the evidence for this state in various processes and the comparison with $f_{0}(1500)$ nearby in mass. In previous surveys supportive [125] and sceptical views [5] have been presented.

\subsection{Production in $p \bar{p}$ annihilation}

The state $f_{0}(1370)$ has been discovered in $p \bar{p}$ annihilation at rest by the Crystal Barrel Collaboration (CBAR) and has been studied together with $f_{0}(1500)$ in the reactions 

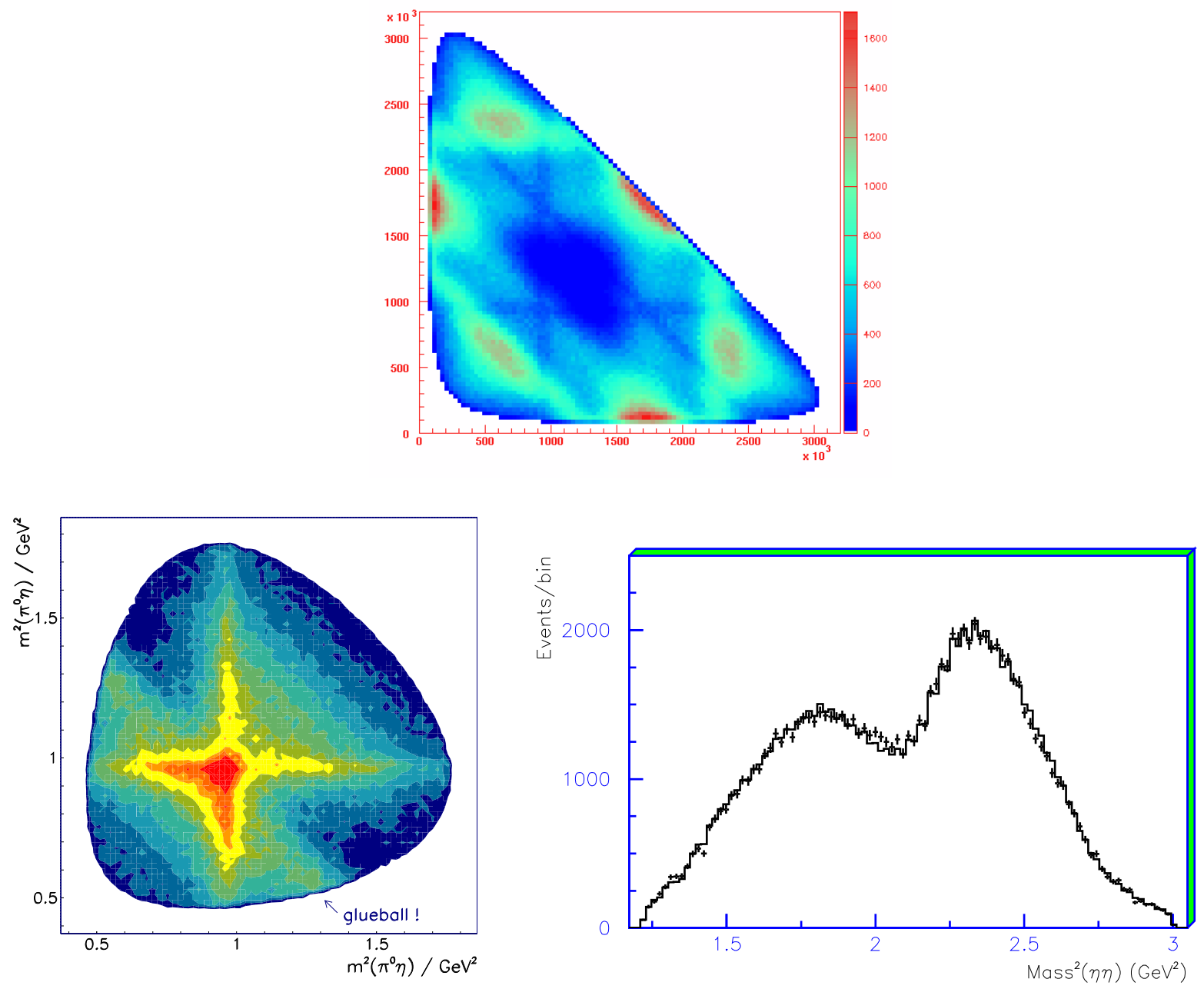

Figure 6. Upper panel: Dalitz plots for $p \bar{p} \rightarrow 3 \pi^{0}$ annihilation at rest; Bottom: Dalitz plot for $p \bar{p} \rightarrow \pi^{0} \eta \eta$ together with $\operatorname{Mass}^{2}(\eta \eta)$ spectrum from Crystal Barrel Collaboration [126] (figures from [5]).

$[126,127,49]$
a) $p \bar{p} \rightarrow \pi^{0} \pi^{0} \pi^{0}$,
b) $p \bar{p} \rightarrow \pi^{0} \eta \eta$,
c) $p \bar{p} \rightarrow \pi^{0} \pi^{0} \eta$.

The analysis is based on the Dalitz plots shown in figure 6 . The $3 \pi^{0}$ Dalitz plot has sixfold symmetry and is based on $\sim 700000$ events. The coloured version of the top plot shows events in bands near the corners at $m^{2}\left(\pi^{0} \pi^{0}\right) \approx 2.25\left(=1.5^{2}\right) \mathrm{GeV}^{2}$ which corresponds to the isotropic decay of the $S$ wave resonance $f_{0}(1500)$ (at fixed (vertical) mass squared $s_{23}$ the (horizontal) $s_{12}$ depends linearly on the decay angle $\cos \theta_{23}$ ). At the mass near $m^{2}=1.370^{2}=1.9 \mathrm{GeV}^{2}$ there is no band of comparable strength, the two bumps at the edges of the plot correspond to $f_{2}(1270)$ with its peaked angular distribution according to spin 2 . The evidence for the second resonance $f_{0}(1370)$ is based on the results of a multi-resonance $K$ matrix fit.

A more direct evidence is suggested from reaction (70b). In the $\pi^{0} \eta \eta$ Dalitz plot (Bottom left of figure 6) one observes the horizontal and vertical bands from $a_{0}(980)$, in the diagonal along the arrow "glueball" there is again a band with flat density related to $f_{0}(1500) \rightarrow \eta \eta$. In parallel, a second band appears to the right (at lower $\eta \eta$ mass), 

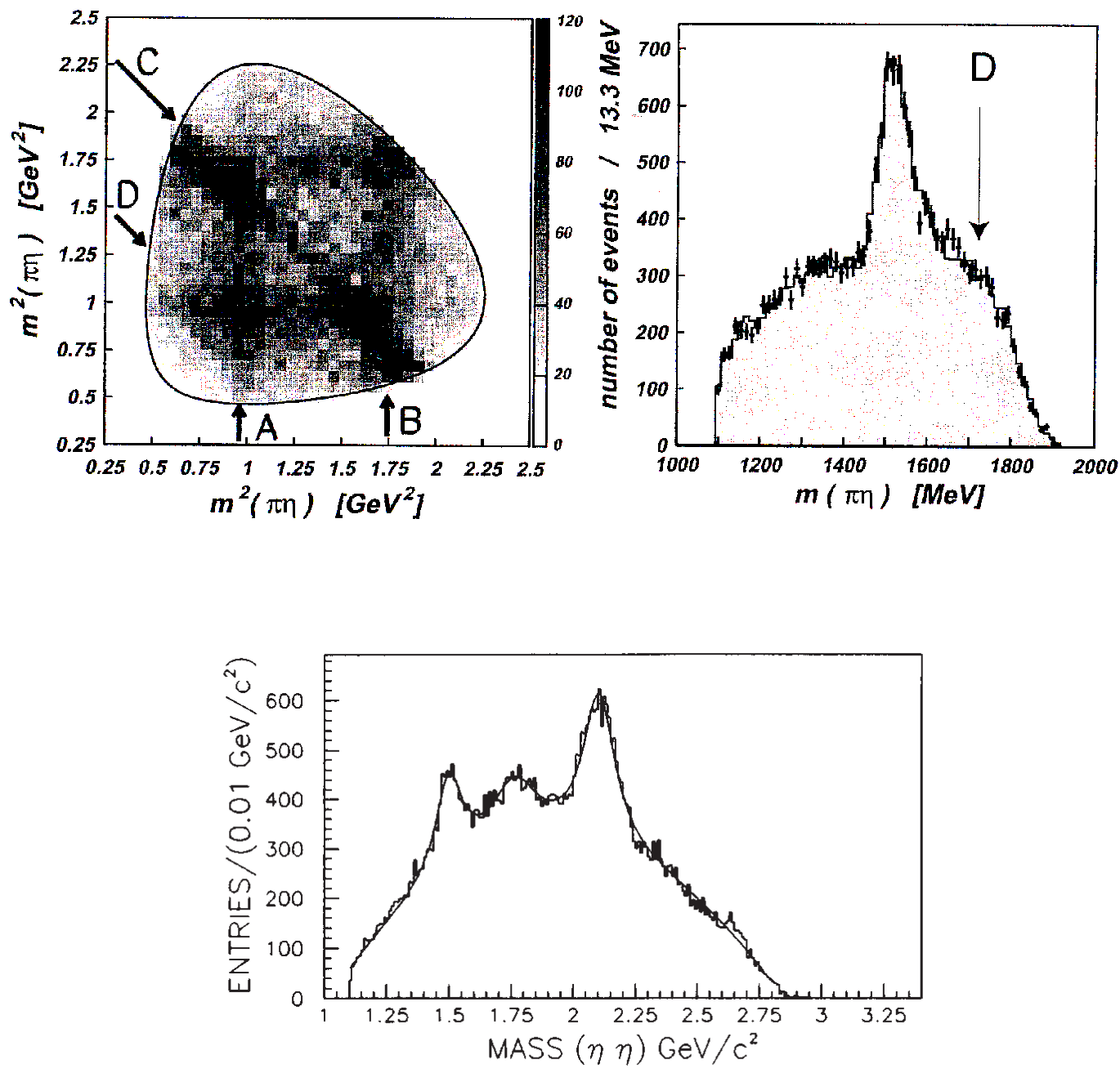

Figure 7. Upper part left: Dalitz plot for $p \bar{p} \rightarrow \pi^{0} \eta \eta$ at $\sqrt{s}=2.0 \mathrm{GeV}$ (Crystal Barrel); right: $\operatorname{mass}^{2}(\eta \eta)$ spectrum (figures from [128]). Bottom: $\eta \eta$ mass spectrum in $p \bar{p} \rightarrow \pi^{0} \eta \eta$ at $\sqrt{s}=3.0 \mathrm{GeV}$, figure from [129] (Experiment E-760).

but the band does not continue to the edges of the plot as the $f_{0}(1500)$ band does. The projection in $m^{2}(\eta \eta)$ (Bottom right of figure 6) shows peaks at higher mass with contributions from $f_{0}(1500)$ and at the lower mass near $m \sim \sqrt{1.7}=1.3 \mathrm{GeV}$, and this peak has been related to $f_{0}(1370)$. The overlap with the $a_{0}(980)$ bands makes it difficult to directly judge the relevance of these peaks and one relies on the fitting procedure.

A clarification can be expected from a change of primary energy which would shift the diagonal bands at fixed $\eta \eta$ mass away from the crossing $\eta \pi^{0}$ bands. Such a measurement has been performed with $900 \mathrm{MeV}$ antiprotons $\left(\sqrt{s_{p \bar{p}}}=2.05 \mathrm{GeV}\right)$ again by CBAR experiment [128]. The $\eta \eta \pi^{0}$ Dalitz plot with two-fold symmetry is shown in figure 7. Indeed, the diagonal band $(\mathrm{C})$ which corresponds to $f_{0}(1500)$ is now shifted away from the crossing $a_{0}(980)$ bands (A). The new bands (B) are related to $a_{2}(1320)$. A second diagonal band, between $f_{0}(1500)$ and the crossing point of the $a_{2}(1320)$ bands would be expected for $f_{0}(1370)$, but there is no indication of any second band. Also 
the peak in the $\eta \eta$ mass spectrum has changed into a shoulder easily related to the resonances in the $\eta \pi^{0}$ channel. It is difficult to invent a mechanism which would make disappear a strong $f_{0}(1370)$ signal visible at $\sqrt{s_{p \bar{p}}}=2 m_{p}$ by increasing the cms energy by only $0.17 \mathrm{GeV}$.

At yet higher energy the process $p \bar{p} \rightarrow \pi^{0} \eta \eta$ has been measured by Experiment E760 at Fermilab [129]. At $\sqrt{s_{p \bar{p}}}=3 \mathrm{GeV}$ the mass spectrum in figure 7 (lower part) again shows a clear peak at $1500 \mathrm{MeV}$ suggestive of $f_{0}(1500)$ (see also discussion in [5]), but the shoulder at low mass has now disappeared entirely and there is no indication of $f_{0}(1370)$. More recently, Experiment E835 has analyzed data on the same reaction at $\sqrt{s_{p \bar{p}}}=3.4 \mathrm{GeV}$ and determined the spins of the observed resonances [130]. As a result they identified their lowest mass peak with $f_{0}(1500)$ and their second peak as $f_{0}(1710)$.

At all energies in these reactions $f_{0}(1500)$ shows up clearly as band in the Dalitz plot and sometimes as peak in mass spectra. On the other hand, no such "direct" evidence is visible for $f_{0}(1370)$. The effect appears only as a result of a global resonance fit at the lowest primary energy where there is a large overlap with other resonance phenomena.

\subsection{Search in phase shift analysis of $\pi \pi$ scattering}

A method which does not rely on parametric global fits is the determination of the complex scattering amplitude in steps of increasing energy directly from the data. Any resonance decaying into the $\pi \pi$ final state has to appear also in the elastic $\pi \pi$ scattering. The scattering amplitude $T_{\ell}(s)=x m_{0} \Gamma(s) /\left(s_{0}-s-i m_{0} \Gamma(s)\right)$ with a Breit-Wigner resonance pole at $s_{0}-i m_{0} \Gamma$ moves along a circle for increasing (real) mass $m=\sqrt{s}$ around $(0, i x / 2)$ with radius $x / 2$ in the complex plane ("Argand diagram"). This circular behaviour is smoothly modified by background effects and it is a characteristic signature of resonant behaviour to look for. The aim of phase shift analysis is the determination of the complex scattering amplitude from the scattering data.

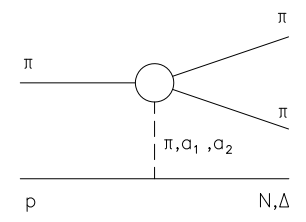

Figure 8. One-Pion-Exchange: $\pi \pi$ scattering contributes to $\pi p \rightarrow \pi \pi N(\Delta)$ reaction.

7.2.1. Methods to reconstruct $\pi \pi$ scattering amplitudes. In this subsection we will recall the main assumptions underlying the reconstruction of the $\pi \pi$ partial wave amplitudes $T_{\ell}$ from measurements of the reaction

$$
\pi^{-} p \rightarrow \pi^{-} \pi^{+} n
$$


(and others) using the One-Pion-Exchange model (OPE) [131] (see figure 8). The incoming pion scatters off a pion from the pion cloud surrounding the target proton at small momentum transfers $t=\left(p_{\text {in }}^{p}-p_{\text {out }}^{N}\right)^{2}$. The theoretical description of the production process is based on Regge theory and the first aim is to isolate the onepion exchange contribution from other exchange contributions (for a review, see [132]). The helicity amplitudes for process (71) in the "t-channel" (Gottfried Jackson) frame (quantization axis along exchanged pion) obtain contributions

$$
f_{\lambda \lambda^{\prime} \mu}^{\ell}=N \frac{\sqrt{2 \ell+1}}{\sqrt{q}} T_{\ell} \delta_{\lambda,-\lambda^{\prime}}\left(m_{\pi \pi} \frac{\sqrt{-t}}{m_{\pi}^{2}-t} \delta_{\mu 0}+\frac{c_{A}}{2} \sqrt{\ell(\ell+1)} \delta_{n, 0}\right)+\ldots
$$

with the $\pi \pi \rightarrow \pi \pi$ partial wave amplitude $T_{\ell}=\sin \delta_{\ell} e^{i \delta_{\ell}}$ as a factor; $N$ is a known normalization, $m_{\pi \pi}$ is the $\pi \pi$ mass and $q=\sqrt{m_{\pi \pi}^{2} / 4-m_{\pi}^{2}}$. The first term with the pole at $t=m_{\pi}^{2}$ corresponds to the OPE Born-term with the pion pair produced with helicity $\mu=0$. The nucleon helicity flip amplitudes with $\lambda=-\lambda^{\prime}$ dominate over non-flip amplitudes at high energies and vanish like $\sqrt{-t}$. Simple models suggest corrections from absorption at small impact parameters of the collision for net helicity flip $n=\left|\lambda-\lambda^{\prime}+\mu\right|=0$, such as the second term in $(72)[133,134,135,136,137]$. Furthermore, contributions from $a_{2}$ exchange are found important at larger $t$ while $a_{1^{-}}$ exchange had been neglected at the time. The determination of the $\pi \pi$ amplitudes $T_{\ell}$ can be based on (72) (see, for example [136]).

Alternatively, one may use $t$-integrated angular distributions $W(\cos \theta, \phi)$, represented by the spherical harmonic moments $\left\langle Y_{L}^{M}\right\rangle$ as function of $m_{\pi \pi}$ which are expressed by the density matrix $\rho$ and helicity amplitudes [137]

$$
\begin{aligned}
\frac{d \sigma}{d m_{\pi \pi} d \Omega} & =N \sum_{J} \sum_{M=-J}^{J}\left\langle Y_{J}^{M}\right\rangle \operatorname{Re} Y_{J}^{M}(\theta, \phi) \\
\left\langle Y_{J}^{M}\right\rangle & =\sum_{\ell, \ell^{\prime}, \mu, \mu^{\prime}} c_{M \mu \mu^{\prime}}^{J \ell \ell^{\prime}} \operatorname{Re} \rho_{\mu \mu^{\prime}}^{\ell \ell^{\prime}} \\
N \rho_{\mu \mu^{\prime}}^{\ell \ell^{\prime}} & =\frac{1}{2} \sum_{\lambda \lambda^{\prime}} f_{\lambda \lambda^{\prime} \mu}^{\ell} f_{\lambda \lambda^{\prime} \mu^{\prime}}^{\ell^{\prime} *}
\end{aligned}
$$

It is useful to introduce amplitudes and the density matrix for asymptotic natural $(+)$ and unnatural exchange $(-)$ which do not interfere in the observables considered here using the parity relations $f_{\lambda \lambda^{\prime} \mu}^{\ell}=(-)^{\lambda+\lambda^{\prime}+\mu} f_{-\lambda-\lambda^{\prime}-\mu}^{\ell}$

$$
\begin{aligned}
& g_{\lambda \lambda^{\prime} \mu}^{\ell( \pm)}=\frac{1}{2}\left(f_{\lambda \lambda^{\prime} \mu}^{\ell} \mp(-)^{m} f_{\lambda \lambda^{\prime}-\mu}^{\ell}\right) \\
& \rho_{\mu \mu^{\prime}}^{\ell \ell^{\prime}( \pm)}=\frac{1}{2}\left(\rho_{\mu \mu^{\prime}}^{\ell \ell^{\prime}} \mp(-)^{m^{\prime}} \rho_{\mu-\mu^{\prime}}^{\ell \ell^{\prime}( \pm)}\right)
\end{aligned}
$$

In general, there are more density matrix elements than measured moments. Motivated by the models for OPE with absorption and amplitudes as in (72) some simplified assumptions for the structure of density matrix elements have been proposed [133]:

(i) "Spin coherence:" There are only nucleon flip amplitudes. In case of experiments with unpolarized target the same consequences on observables follow already from the 
proportionality between flip and non-flip amplitudes

$$
g_{++\mu}^{\ell( \pm)}=\alpha^{( \pm)} g_{+-\mu}^{\ell( \pm)} .
$$

This relation also implies for the density matrices $\operatorname{Rank}\left(\rho^{( \pm)}\right) \leq 1$ while $\operatorname{Rank}\left(\rho^{( \pm)}\right) \leq 2$ would be allowed for in general. Then one can also write

$$
\rho_{\mu \mu^{\prime}}^{\ell \ell^{\prime}( \pm)}=\tilde{g}_{\mu}^{\ell( \pm)} \tilde{g}_{\mu^{\prime}}^{\ell( \pm) *}
$$

with $\tilde{g}_{\mu}^{\ell( \pm)}=\sqrt{1+\left|\alpha^{( \pm)}\right|^{2}} g_{+-\mu}^{\ell( \pm)}$. At this point the sum over nucleon helicities has disappeared and the relation between the observable moments and the $\pi \pi$ production amplitudes is very similar to on shell $\pi \pi$ scattering and one uses for the amplitudes $\tilde{g}_{\mu}^{\ell( \pm)}$ the spectroscopic notation $L_{\mu}^{( \pm)}=S_{0}^{(-)}, P_{0}^{(-)}, P_{1}^{( \pm)} \ldots$

(ii) "Phase coherence:" A second simplification suggested by absorbed OPE (72) is the appearance of the same phase $\delta_{\ell}$ for all helicity amplitudes with the same $\pi \pi$ spin $\ell$.

These two assumptions are motivated only for small $t$; they could be violated by the presence of other exchanges like $a_{1}$ or $a_{2}$ exchanges. Both assumptions together actually lead to constraints between the observable moments $\left\langle Y_{L}^{M}\right\rangle$ which are rather well satisfied experimentally at $17 \mathrm{GeV} \pi p$ collisions [101]. Also the reduction in rank of the density matrix has been confirmed experimentally near the $\rho$ meson mass peak [138].

Alternatively, one can relax the assumption on phase coherence and introduce extra phases between the different helicity amplitudes [134]. Measurements of relative phases, such as $\Delta^{\ell}=\delta_{1}^{\ell(-)}-\delta_{0}^{\ell(-)}$, can check the quality of the phase coherence approximation and the dominance of a single production mechanism for spin $\ell$. Further discussions of the two-pseudoscalar system and the ambiguity problems are given in [139].

As an example we give the relations for identical particles with even angular momentum $[101,139]$.

$$
\begin{aligned}
& \sqrt{4 \pi}\left\langle Y_{0}^{0}\right\rangle=\left|S_{0}^{-}\right|^{2}+\left|D_{0}^{-}\right|^{2}+\left|D_{1}^{-}\right|^{2}+\left|D_{1}^{+}\right|^{2} \\
& \sqrt{4 \pi}\left\langle Y_{2}^{0}\right\rangle=\frac{\sqrt{5}}{7}\left(2\left|D_{0}^{-}\right|^{2}+\left|D_{1}^{-}\right|^{2}+\left|D_{1}^{+}\right|^{2}\right)+2\left|S_{0}^{-}\right|\left|D_{0}^{-}\right| \cos \left(\phi_{S_{0}^{-}}-\phi_{D_{0}^{-}}\right) \\
& \sqrt{4 \pi}\left\langle Y_{2}^{1}\right\rangle=\frac{\sqrt{10}}{7}\left|D_{1}^{-}\right|\left|D_{0}^{-}\right| \cos \left(\phi_{D_{1}^{-}}-\phi_{D_{0}^{-}}\right)+\sqrt{2}\left|S_{0}^{-}\right|\left|D_{1}^{-}\right| \cos \left(\phi_{S_{0}^{-}}-\phi_{D_{1}^{-}}\right) \\
& \sqrt{4 \pi}\left\langle Y_{4}^{0}\right\rangle=\frac{6}{7}\left|D_{0}^{-}\right|^{2}-\frac{4}{7}\left(\left|D_{1}^{-}\right|^{2}+\left|D_{1}^{+}\right|^{2}\right) \\
& \sqrt{4 \pi}\left\langle Y_{4}^{1}\right\rangle=\frac{2 \sqrt{15}}{7}\left|D_{0}^{-}\right|\left|D_{1}^{-}\right| \cos \left(\phi_{D_{0}^{-}}-\phi_{D_{1}^{-}}\right)
\end{aligned}
$$

We stress again, that this kind of equations without reference to nucleon helicities rely on the assumption of "spin coherence" for unpolarized target experiments,

The validity of the results obtained from production experiments based on the OPE model has been demonstrated recently by the nice agreement between $K \pi$ phase shifts obtained from $K p \rightarrow K \pi n$ and from decays $D \rightarrow K \pi e \nu_{e}$ using the Watson theorem (see subsection 5.2 and figure 5).

7.2.2. Results on $\pi^{+} \pi^{-} \rightarrow \pi^{+} \pi^{-}$. A measurement of $\pi^{-} p \rightarrow \pi^{-} \pi^{+} n$ with unpolarized target has been carried out by the CERN-Munich Collaboration [137]. They measured 
the moments $\left\langle Y_{L}^{M}\right\rangle$ in the range $0.6<m_{\pi \pi}<1.8 \mathrm{GeV}$ from which the partial waves with $\pi \pi$ spin $\ell \leq 3$ have been determined. The overall phase of the amplitude cannot be determined directly but it is inferred from the Breit-Wigner phase of the leading resonances $\rho(770), f_{2}(1270)$ and $\rho_{3}(1690)$. In general, the moments with $L \leq L_{\max }$ determine the partial wave amplitudes only up to discrete ambiguities. Writing the amplitude $T(s, z)=f(s) \Pi_{i=1}^{L_{\max } / 2}\left(z-z_{i}\right)$ as function of $z=\cos \theta$ one notes that the measurement of $|T(s, z)|^{2}$ at fixed $s=m_{\pi \pi}^{2}$ does not determine the sign of the imaginary part of the zeros $z_{i}$ ("Barrelet zero" [140]) and so one obtains $2^{L_{\max } / 2}$ different solutions. Some can be removed by unitarity constraints. Subsequently, an experiment with polarized target has been carried out by the CERN-Krakow-Munich Collaboration $[141,142]$. The following results have been presented on $\pi \pi$ scattering amplitudes $T_{\ell}$ above $1 \mathrm{GeV}$ which are of interest for our discussion of $f_{0}(1370)$.

(i) The first analysis by Hyams et al. [101] determined the moments averaged over $|t|<0.15 \mathrm{GeV}$ (CM-I). These moments have been fitted by amplitudes $g_{\mu}^{\ell( \pm)}(\mu=0,1)$ as in (73)-(79) parameterizing the $\pi \pi$ amplitudes as function of $m_{\pi \pi}$ by a $K$ matrix. In addition, local deviations from this solution have been studied. The $S$ wave featured the narrow $f_{0}(980)$ interfering with a broad background passing $90^{\circ}$ near $m_{\pi \pi} \approx 1 \mathrm{GeV}$. A classification in terms of amplitude zeros has been given but no study of alternative solutions has been attempted.

(ii) The first full study of ambiguities from CM-I data has been carried out by Estabrooks and Martin $[143,144]$. They obtained four different phase shift solutions A-D classified according to the Barrelet zeros. A $K$ matrix fit to the $\mathrm{S}$ waves of the four solutions and taking into account $K \bar{K}$ data as well, yielded three scalar resonances $f_{0}(800), f_{0}(1005)$ and $f_{0}(1540)$ with width around 1000, 8 and $200 \mathrm{MeV}$ resp. [118]. A subsequent analysis in which the phase of the amplitude has been determined using the analyticity constraints of dispersion relations produced modified solutions $\alpha, \beta\left(\beta^{\prime}\right)$ starting from A,B [145]. Because of earlier $\pi^{0} \pi^{0}$ data the solution $\mathrm{C}$ as starting solution has been rejected at the time but has been favoured later.

(iii) A second analysis by the CERN-Munich group [146] (CM-II) included in the fits to the primary data a complete error correlation matrix using larger mass bins and obtained in that way $\pi \pi$ amplitudes more strongly constrained than those of [101]. Also in these fits some $t$ dependence is parameterized. The ambiguities are analyzed and again 4 solutions are identified. The smaller error bars of zeros $\operatorname{Im} z_{i}$ do not allow the crossing of $\operatorname{Im} z_{1}$ at $1.5 \mathrm{GeV}$ as in the previous analysis (i) in [101]; therefore these earlier results should not be trusted above $\sim 1.4 \mathrm{GeV}$.

(iv) A further experiment with transversely polarized target has been performed by the CERN-Krakow-Munich Collaboration [141, 142] with the aim to check the assumptions underlying the previous experiments. Large polarization effects have been found which prove the existence of sizable nucleon non-flip amplitudes of $a_{1}$-exchange type, so far neglected. However, the more general property of "spin coherence" is still approximately valid, i.e. amplitudes with nucleon spin flip and non-flip are proportional for different di-meson spin orientations $\ell, \mu$ to a good approximation in the average over 


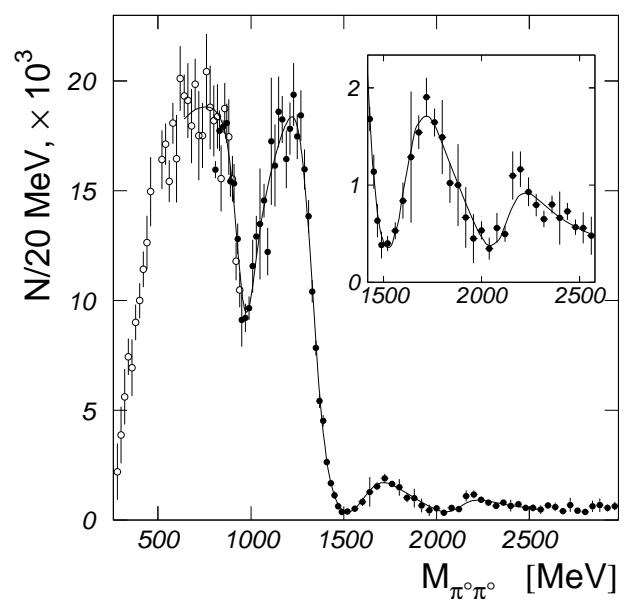

Figure 9. Dependence of $S$ wave intensity on $\pi^{0} \pi^{0}$-mass in $\pi^{-} p \rightarrow \pi^{0} \pi^{0} n$. The first two dips reflect the destructive interference of a broad background with the narrow $f_{0}(980)$ and $f_{0}(1500)$, figure from [150] (GAMS Collaboration).

$t$ as in (78), so that the previous analyses can be maintained (see also discussion in [61]). In [147] it has been shown that the $\pi \pi S$ wave phases in the region 1000-1400 $\mathrm{MeV}$ agree quite well from the polarized target experiment [148], from analysis (i) [101] and solutions $(---)$ or $(-+-)$ from [146], corresponding to solutions A,C in [144]. We therefore consider only these two solutions in the following.

7.2.3. Results on $\pi^{+} \pi^{-} \rightarrow \pi^{0} \pi^{0}$. Partial wave amplitudes of the charge exchange reaction have been obtained from the production process $\pi^{-} p \rightarrow \pi^{0} \pi^{0} n$ at incident momentum 18.3 GeV by experiment E853 at the Brookhaven National Lab [149] and at $100 \mathrm{GeV}$ by the GAMS Collaboration at CERN [150]. Both experiments measured the moments integrated over small $|t|$ and determined the partial wave amplitudes using methods as discussed above. The E853 experiment found for $m_{\pi \pi} \lesssim 1.5 \mathrm{GeV}$ one solution with phase coherence $\left(\phi_{D_{0}}-\phi_{D_{-}} \approx 0\right)$ and another one with large relative phase; above that mass the errors are rather large. Also at larger $-t>0.4 \mathrm{GeV}^{2}$ the phase coherent solutions disappeared. GAMS found solutions with phase coherence in the full mass range studied up to $3 \mathrm{GeV}$ and imposed this condition subsequently in the phase shift analysis. The mass spectrum of the $S$ wave is shown in figure 9 .

7.2.4. Selection of a unique isoscalar $\pi \pi S$ wave. A further selection between the two phase shift solutions $(---)$ and $(-+-)$ or A and $\mathrm{C}$, respectively, left over from the $\pi^{+} \pi^{-}$analysis can be obtained by comparing to $\pi^{+} \pi^{-} \rightarrow \pi^{0} \pi^{0}$ with its different ambiguity structure. Comparing CERN-Munich and GAMS data which have good accuracy up to $m_{\pi \pi} \leq 1.8 \mathrm{GeV}$ a unique solution has been established $[102,151] . \ddagger \mathrm{A}$ $\ddagger$ These analyses are preliminary concerning the parametric $S$ matrix fits. 

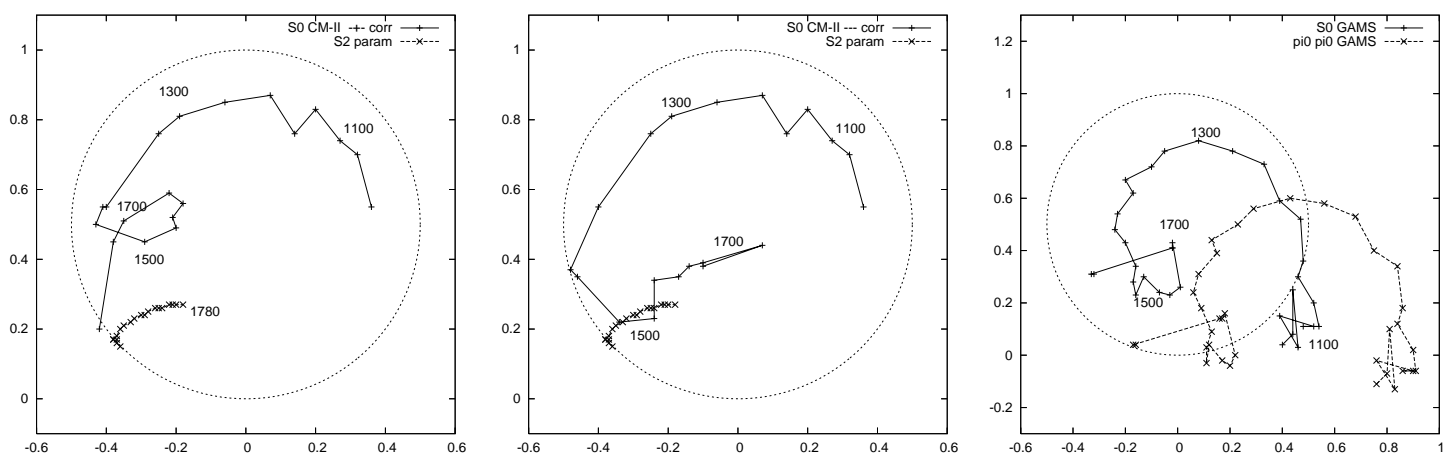

Figure 10. Argand diagrams for $\pi \pi S_{I}$ waves: $S_{0}$ solutions $(-+-)$ and $(---)$ from $\pi^{+} \pi^{-} \rightarrow \pi^{+} \pi^{-}$(CM-II) but using new inelastic $S_{2}$ as shown; right panel: $S_{00}$ amplitude for $\pi^{+} \pi^{-} \rightarrow \pi^{0} \pi^{0}$ (GAMS) and component $S_{0}$ thereof (figures from [102]).

new (inelastic) $I=2$ amplitude $S_{2}$ for the mass range $1000 \leq m_{\pi \pi} \leq 1800 \mathrm{MeV}$ has been constructed from data $[152,153]$ and has been used to obtain updated $I=0$ amplitudes $S_{0}$ (see Argand diagrams left in figure 10 for new $S_{0}$ and $S_{2}$ ). The diagram rightmost in figure 10 shows the $S$ wave amplitude $S_{00}$ for the process $\pi^{+} \pi^{-} \rightarrow \pi^{0} \pi^{0}$ which has been reconstructed from the magnitude $\left|S_{00}\right|^{2}$, in the given normalization, the phase $\left|\phi_{S}-\phi_{D_{0}}\right|$ and the Breit Wigner phase and normalization of $f_{2}(1270)$ in the $D_{0}$ wave. The $I=0$ amplitude $S_{0}$ is obtained from $S_{00}=S_{0}-S_{2}$ and is shown in the same diagram (normalization $\left|S_{I}-\frac{i}{2}\right| \leq \frac{1}{2}$ ).

The amplitude $S_{0}$ obtained from $\pi^{0} \pi^{0}$ data should agree with $S_{0}$ from $\pi^{+} \pi^{-}$. This is only true approximately for the $(-+-)$ solution: there is a circular motion from 1000 to $1740 \mathrm{MeV}$ with a smaller circle superimposed above $1400 \mathrm{MeV}$. Solution ( - - - ) would turn into the center of the circle instead and so it has been rejected. There are discrepancies between the remaining solutions $S_{0}$ which have been related to systematic uncertainties in the overall phase, the $I=2$ amplitude and influence from higher partial waves. These uncertainties over large mass scales should not affect the nature of the local resonance phenomena like $f_{0}(1370)$ and $f_{0}(1500)$.

The amount of agreement is nevertheless impressive, in particular concerning the existence of $f_{0}(1500)$ which shows up clearly as a small circle in both analyses. Mass and width are roughly compatible with the PDG values [4] $(\mathrm{m}=1505 \mathrm{MeV}, \Gamma=109 \mathrm{MeV})$. The partial width $x_{\pi \pi}=\Gamma_{\pi \pi} / \Gamma_{t o t}$ in the resonant amplitude $T_{0}=x_{\pi \pi} m_{0} \Gamma /\left(s_{0}-s-i m_{0} \Gamma\right)$ can be estimated from the depth of the $f_{0}(1500)$ circle in figure 10 (CM-II), see also inelasticity $\eta_{0}^{0}$ in figure $11\left(\Delta x=2 \Delta \eta_{0}^{0}\right)$ :

$$
f_{0}(1500): \quad x_{\pi \pi}=0.25 \pm 0.05(\mathrm{CM}-\mathrm{II}), \quad x_{\pi \pi}=0.349 \pm 0.023(\mathrm{PDG}) .
$$

Here the first result (CM-II) is determined from $\operatorname{Im} T_{0}$ of the resonant elastic partial wave amplitude and the second one (PDG) from all inelastic channel cross sections; both should agree because of the optical theorem and they roughly do within $30 \%$.

In order to represent the selected solution $S_{0}(-+-)$ in terms of a minimal number of resonances an ansatz for a unitary 3-resonance $S$ matrix for the 3 channels $(\pi \pi, K \bar{K}, 4 \pi)$ 

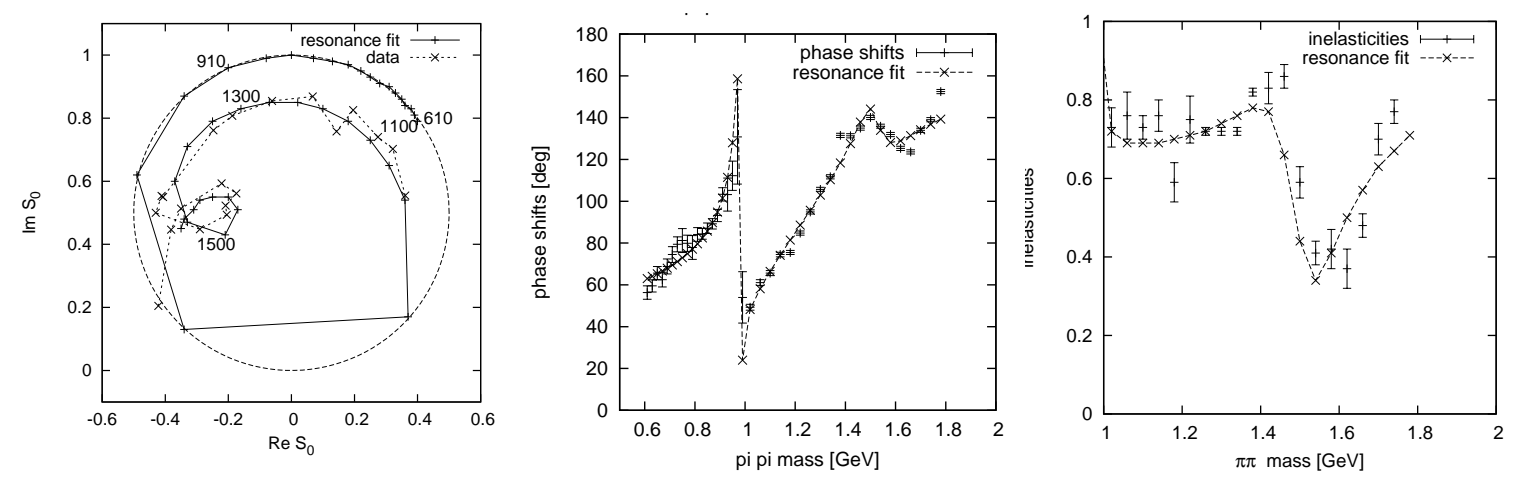

Figure 11. Resonance fit (83) in comparison with data (CM-I/II): Argand diagram for corrected $S_{0}$ wave, phase shift $\delta_{0}^{0}$ and inelasticity $\eta_{0}^{0}$ (figures from [102]).

has been fitted to the CM-II data. In a generalization of (46) the $S$ matrix is written as product of three unitary matrices for resonances $S_{R}=1+2 i T_{R}$

$$
\begin{aligned}
S= & S_{f_{0}(980)} S_{f_{0}(1500)} S_{\text {broad }} \\
T_{R}=\left[m_{0}^{2}-m_{\pi \pi}^{2}-i\left(\rho_{1} g_{1}^{2}+\rho_{2} g_{2}^{2}+\rho_{3} g_{3}^{2}\right)\right]^{-1} & \\
& \quad \times \rho^{\frac{1}{2} T}\left(g_{i} g_{j}\right) \rho^{\frac{1}{2}}
\end{aligned}
$$

where $\rho_{i}=2 k_{i} / \sqrt{s}$ and $\left(g_{i} g_{j}\right)$ is the matrix with real constant couplings. This $S$ matrix behaves locally like a superposition of a narrow inelastic resonance over a largely elastic background as in (46), but it does not fulfill the symmetry requirements for the $S$ matrix exactly, therefore further tests are necessary. Also, the formulae $(82),(83)$ do not represent the correct behaviour near $\pi \pi$ threshold and cannot be used for the determination of the pole of $S_{\mathrm{broad}}$.

These formulae have been fitted in [102] to the data from CM-II for $m_{\pi \pi}>1000$ $\mathrm{MeV}$ and the phases from CM-I in $600<m_{\pi \pi}<1000 \mathrm{MeV}$ for definiteness. As can be seen in figure 11 the three resonances give a good overall description of the data. The broad state corresponds to a Breit-Wigner resonance with mass parameter $m_{0}=1100$ $\mathrm{MeV}$ and a total width of similar size which we relate to the high mass tail of $f_{0}(500)$.

7.2.5. Search for $f_{0}(1370)$ in $\pi \pi$ scattering. The fit in figure 11 reproduces the main features related to the rapid phase variations for the two narrow resonances at 1000 and $1500 \mathrm{MeV}$ above a broad background which can also be described as resonant state. In particular, there is no indication of an additional state around $1370 \mathrm{MeV}$ which should become noticeable as an extra circle and the related variations in phase and inelasticity comparable to $f_{0}(1500)$. If we estimate from figure 11 that an extra state with more than $1 / 3$ of elasticity $x_{\pi \pi}$ of $f_{0}(1500)$ should have been noticed, we arrive at the limit

$$
f_{0}(1370): \quad x_{\pi \pi}<0.1 \quad(\mathrm{CL}=95 \%) \quad(\mathrm{CM}-\mathrm{II} \text { data })
$$

The Argand diagram of solution $\mathrm{C}$ in [143] shows a similar behaviour. The $\mathrm{K}$ matrix fits to those data require $f_{0}(1500)$ but no additional resonance near $1300 \mathrm{MeV}$ [118]. 
It should be noted that in figure 11 the data points represent in each mass interval, together with the results from the other partial waves an (almost) perfect fit to the original $\left\langle Y_{L}^{M}\right\rangle$ data at this mass. There is no correlation between data points at different masses. An additional resonance should therefore become visible in such energy independent analyses and the results in (83),(84) don't depend on the parameterization chosen for the fit.

The present evidence for $f_{0}(1370)$ comes almost exclusively from fitting energy dependent parameterizations to the angular moments or Dalitz plots. For example, Bugg [125] has fitted the CM-I moments (and other data) to a superposition of resonances with a sizable $f_{0}(1370)$ contribution in $\pi \pi$ scattering. The $\pi \pi S$ wave amplitude shows an extra circle between 1000 and $1500 \mathrm{MeV}$. From the size of this circle in comparison to the elastic circle below $1000 \mathrm{MeV}$ we conclude that the elastic width of $f_{0}(1370)$ in this fit is about $x_{\pi \pi} \sim 0.35$ (figure 16 in [125]). Such an effect can hardly be consistent with the $\eta-\delta$ "data" in figure 11 which in turn fit the original moments in all mass bins separately. Accordingly, although the overall fit to the moments (figures 14,15 in [125]) looks reasonable, there are significant local deviations between fit and data in all moments, also in the region around $1300 \mathrm{MeV}$.

In the $\mathrm{K}$ matrix analysis by Anisovich and Sarantsev [37] $f_{0}(1370)$ appears with partial width $x_{\pi \pi} \sim 0.19-0.26$, depending on the solution (Table 6 in [37]), and it is larger or equal to the $\pi \pi$ width of $f_{0}(1500)$ with $x_{\pi \pi} \sim 0.19-0.23$. Decay widths into $\pi \pi$ of both states with equal strength are hard to reconcile with the energy-independent result (84) from CM-II and the amplitude structure in figure 11.

The GAMS Collaboration has performed a partial wave decomposition of their $\pi^{0} \pi^{0}$ data [150] which has been used to reconstruct the $S$ wave amplitudes (our figures 10 and 11 from [102]). The Collaboration also determined the contributions of individual resonances to the $S$ wave mass spectrum $|S|^{2}$ (figure 9). A model amplitude is fitted to the spectrum which represents a superposition of a real Gaussian-type background and Breit-Wigner resonances with arbitrary production phases. With the following production cross sections $\sigma \times B R$ a good description has been obtained (see curves in figure 9)

$$
f_{0}(980): 5.4 \pm 1.2 \mathrm{nb}, f_{0}(1300): 70 \pm 15 \mathrm{nb}, f_{0}(1500): 12 \pm 3 \mathrm{nb}, f_{0}(2010): 3 \pm 1 \mathrm{nb}(85)
$$

The fit requires the by far largest cross section for $f_{0}(1300)$ which is not at all visible in the amplitude analysis of our figure 10. This conflict arises using a fitting procedure which tries to derive a two-dimensional (complex) amplitude with several interfering components from a one-dimensional mass spectrum which ends up with ambiguities. One can anticipate that the model amplitude for the $S$ wave would not fit the relative phases to the $D$ wave represented by the $f_{2}(1270)$ resonant amplitude which enter the amplitude analysis. It should also be noted that the second dip near $1500 \mathrm{MeV}$ in the $S$ wave mass spectrum is generated not only by $f_{0}(1500)$ but also by the large background from the isotensor $S_{2}$ (see figure 10). 
7.2.6. Inelastic scattering $\pi \pi \rightarrow K \bar{K}, \eta \eta$. Data on $\pi \pi \rightarrow K \bar{K}$ have been analyzed as well. The experiments with highest statistics are :

(i) Argonne experiment on $\pi^{-} p \rightarrow K^{-} K^{+} n$ and $\pi^{+} n \rightarrow K^{-} K^{+} p$ at $6 \mathrm{GeV}$ for $m_{K \bar{K}}<1.55 \mathrm{GeV}$ by Cohen et al. [92]. Their complete analysis yields 8 different solutions; they are all eliminated but one. They find one $\mathrm{S}$ wave resonance with $m_{0}=1425 \pm 15 \mathrm{MeV}$ and width $\Gamma=160 \pm 30 \mathrm{MeV}$. From a determination of the zeros of Det $\left(K^{-1}\right)$ they exclude the existence of more than one resonance below 1500 $\mathrm{MeV}$. A peak in the mass spectrum near $1300 \mathrm{MeV}$ associated with a smooth phase behaviour is explained by the interference between the background from $f_{0}(980)$ and the resonance at $1425 \mathrm{MeV}$ (figure 33 of [92]). Their Argand diagram suggests a negative relative coupling between background and resonance, as also concluded in [61] for the Brookhaven experiment.

(ii) Brookhaven experiment on $\pi^{-} p \rightarrow K_{s}^{0} K_{s}^{0} n$ at $23 \mathrm{GeV}$ and for $m_{K \bar{K}}<2.4 \mathrm{GeV}$ by Etkin et al. [91]. Their best fit includes a resonance at $m_{0}=1463 \pm 90 \mathrm{MeV}$ and width $\Gamma=118 \pm_{-16}^{+138}$ in perfect agreement with $f_{0}(1500)$ from PDG. Their global fit with $f_{0}(980)$ and $f_{0}(1500)$ (but without $f_{0}(500)$ ) represents the $S$ wave phase $\delta_{0}^{0}$ well, but not the peak near $1300 \mathrm{MeV}$.

These results are summarized by Büttiker et al. [154] in figure 12. The phase shifts at the $K \bar{K}$ threshold join smoothly with those from elastic $\pi \pi$ scattering which coincide below threshold because of unitarity (see, for example [155]). Near the $K \bar{K}$ threshold the presence of a $P$ wave - the tail of the $\rho$ meson - in $\pi^{+} \pi^{-} \rightarrow K^{+} K^{-}$(Cohen et al.) allows the accurate determination of the $S$ wave. As there are conflicting results in this region a preference is given for low energies $E<1.3 \mathrm{GeV}$ to the data by Cohen et al. The $\pi \pi$ phase shifts obtained in a dispersion relation approach [100] also prefer the upper branch of phases which approach $225^{\circ}$ at $K \bar{K}$ threshold.

Subsequent descriptions in terms of resonances including the broad object $f_{0}(500)$ yield a good qualitative description of $\pi \pi$ and $K \bar{K}$ data without an additional resonant state near $1300 \mathrm{MeV}$ : a K matrix fit [118] with three poles and a fit with the ansatz (83) [151]. On the other hand, a contribution from $f_{0}(1370)$ is suggested in [90] with an effect hardly visible in the phase motion. Indeed, the phase behaves very smoothly around $1370 \mathrm{MeV}$ in the data (Left panel of figure 12), while there is a strong effect from $f_{0}(1500)$ similarly to the elastic $\pi \pi$ channel, and, of course, from $f_{0}(980)$.

(iii) Finally, the inelastic process $\pi^{+} \pi^{-} \rightarrow \eta \eta$ has been measured by Binon et al. (GAMS Collaboration)[156] (more recent results in [157]). Also in this case, a significant effect is seen for $f_{0}(1500)$ with mass and width consistent with PDG. The state interferes with the broad background, opposite in sign to $K \bar{K}$ [61]. It forms a circle in the Dalitz plot also seen in [90]. A clearly visible signal from $f_{0}(1370)$ can hardly be identified.

\subsection{Decays of $D$ and $B$ into $f_{0}$ mesons}

In the weak decays of heavy quark mesons some well defined $q \bar{q}$ states evolve from the intermediate weak and strong interaction processes and they finally can form isoscalar 

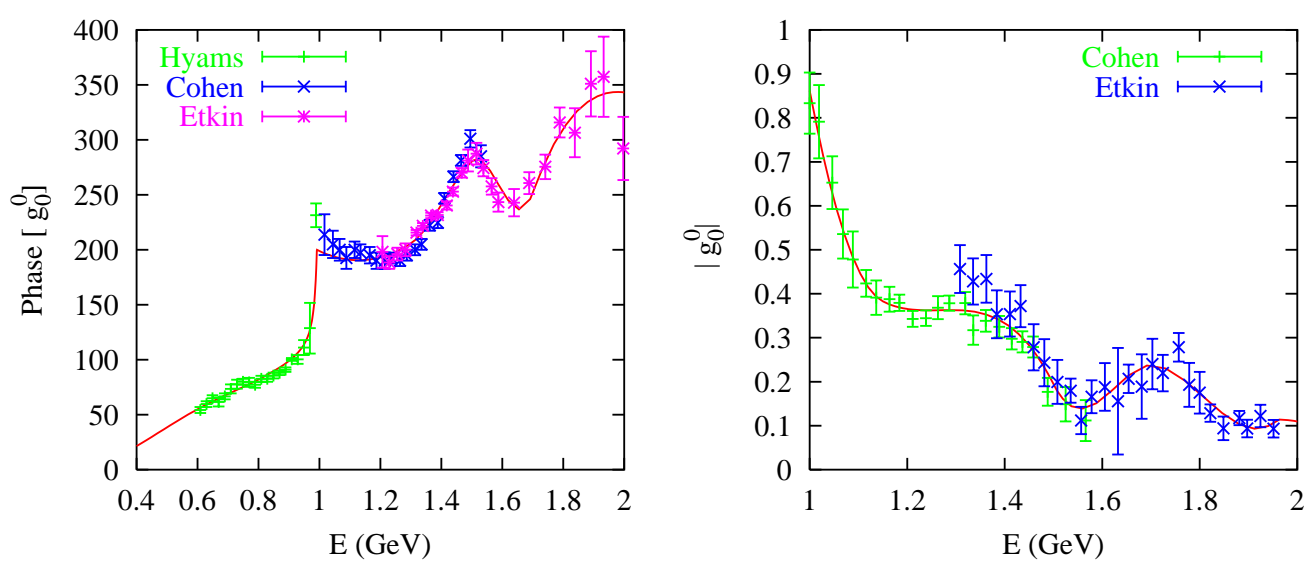

Figure 12. Phase and magnitude of $I=0 S$ wave amplitude for $\pi \pi \rightarrow K \bar{K}$ vs. $E_{\pi \pi}$ (data [101, 92, 91]); curves for $E_{\pi \pi}>1 \mathrm{GeV}$ are polynomial fits (figure from [154]).
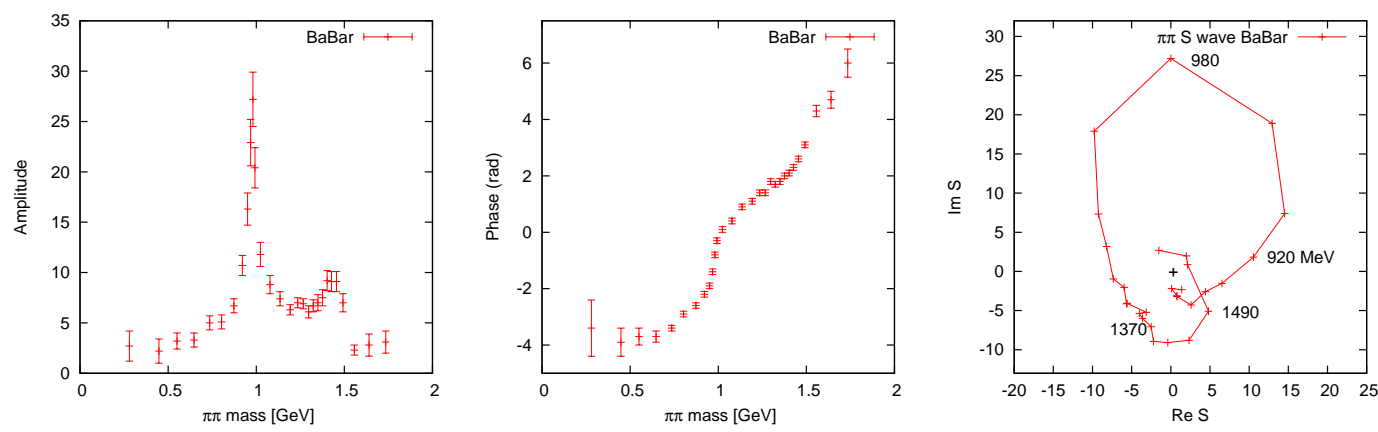

Figure 13. $\pi \pi S$ wave amplitude and phase extracted from decays $D_{s}^{+} \rightarrow \pi^{+} \pi^{-} \pi^{+}$ (BaBar Collaboration [122]); right panel: Argand diagram for $\pi \pi$ amplitude (the phase is normalized to $\pi / 2$ at the $f_{0}(980)$ peak). The points are not equidistant in mass.

mesons. Then, these processes may help to clarify their intrinsic structure.

7.3.1. Decay of $D_{s}$ mesons. The dominant subprocess for $f_{0}$ production in the decay $D_{s}^{+} \rightarrow \pi^{+} \pi^{-} \pi^{+}$is identified as

$$
D_{s}^{+} \rightarrow \pi^{+}+s \bar{s} ; \quad s \bar{s} \rightarrow f_{0} \rightarrow \pi^{+} \pi^{-}
$$

Earlier studies of this process by E791 [158] and FOCUS [159] Collaborations have shown a strong signal from $f_{0}(980)$ and some resonant structure associated to $f_{0}(1370)$ or $f_{0}(1500)$. In a dedicated analysis by Klempt, Matveev and Sarantsev [160] of E791 data no positive evidence for $f_{0}(1370)$ besides $f_{0}(1500)$ was found, although such a contribution has not been excluded.

In a more recent high statistics investigation of the decay $D_{s} \rightarrow 3 \pi$ by the BaBar Collaboration [122] the known resonances $\rho, f_{2}$ have been represented by Breit-Wigner forms while the $S$ wave amplitude $A_{S}$ is parameterized in a model independent way by 
an interpolation between 30 points in the complex plane $\S$

$$
A_{S}\left(m_{\pi \pi}\right)=\left.\operatorname{Interp}\left(c_{k}\left(m_{\pi \pi}\right) e^{i \phi_{k}\left(m_{\pi \pi}\right)}\right)\right|_{k=1 \ldots 30}
$$

The resulting amplitudes and phases are shown in figure 13. One observes clearly the peak from $f_{0}(980)$ and another peak below $1500 \mathrm{MeV}$. The phases show two regions of strong variation with mass, near $1000 \mathrm{MeV}$ and near $1500 \mathrm{MeV}$ (middle panel). Near $1370 \mathrm{MeV}$ the phase motion per mass interval is near minimum, without resonant signal.

The Argand diagram (right panel) shows a large circle peaking at $980 \mathrm{MeV}$ with a small circle near $1500 \mathrm{MeV}$ superimposed curving outward. This behaviour corresponds to a negative relative coupling of $f_{0}(1500)$ with respect to the background from $f_{0}(980)$ (and some $f_{0}(500)$ ) according to formula (46) with $T \simeq T_{B}-T_{R} e^{2 i \delta_{B}}$.

This is to be contrasted to elastic $\pi \pi$ scattering in figure 11 where the small circle at $1500 \mathrm{MeV}$ is curving inward corresponding to positive coupling. The same situation with different sign of coupling has been discussed before in $\pi \pi \rightarrow K \bar{K}$ and $\pi \pi \rightarrow \eta \eta[61]$ and can be interpreted in terms of the near flavour octet composition of $f_{0}(1500)$ as in route 2 according to (34). There is no evidence for an extra circle in the Argand plot near 1370 $\mathrm{MeV}$. However, as the initial coupling to $s \bar{s}$ is not known one cannot derive a limit on the branching fraction of $f_{0}(1370) \rightarrow \pi \pi$. The shift of the $f_{0}(1500)$ peak towards smaller masses is due to the interference with the background amplitude from $f_{0}(980) / f_{0}(500)$. The negative relative coupling of $f_{0}(980)$ and $f_{0}(1500)$ to $s \bar{s}$ is also reflected by the clear depletion in the Dalitz plot of $D_{s} \rightarrow 3 \pi$ at the $f_{0}(980)-f_{0}(1500)$ band crossing [122], and this has been seen already in the data from the E791 Collaboration [161], see also the discussion of phases in [70].

7.3.2. Decay $B^{+} \rightarrow K^{+} f_{0}, f_{0} \rightarrow \pi \pi, K \bar{K}$. The $\pi \pi$ and $K \bar{K}$ mass spectra from these decays as measured by Belle [82] are shown in figure 14. There is a clear signal of $f_{0}(1500)$ in the $K^{+} K^{-}$spectrum, but not in $\pi^{+} \pi^{-}$, despite the 4 times larger decay probability in favour of the $\pi \pi$ channel. Therefore, a new state $f_{X}(1500)$ has been suggested [82] which looks like $f_{0}(1500)$ except for the branching ratios.\|

This effect can also be explained [48] by taking into account the different quark composition of $f_{0}(1500)$ near flavour octet as in (35) and $f_{0}(500)$ (or $f_{0}(980)$ ) taken as flavour singlet (or glueball) along route 2 which leads to an opposite sign of interference in the strange and non-strange components: one can obtain a constructive interference of both components in $K^{+} K^{-}$and then one finds a destructive one in $\pi^{+} \pi^{-}$. In this model represented by the curves in figure 14 the broad background from $f_{0}(500)$ and

$\S$ This method has been used first by the Fermilab E791 Collaboration [158].

$\|$ In the analysis of $B \rightarrow K \bar{K} K$ by BaBaR [162] the mass peak near $1500 \mathrm{MeV}$ has been fitted by $X(1550)$ but with a large width of $257 \pm 33 \mathrm{MeV}$, twice as large as of $f_{0}(1500)$. We note however, that the fit - contrary to the analysis [48] - corresponds to an approximately destructive interference of this resonance with background. The data are not so well reproduced in the resonance region and another solution with smaller width may exist. 

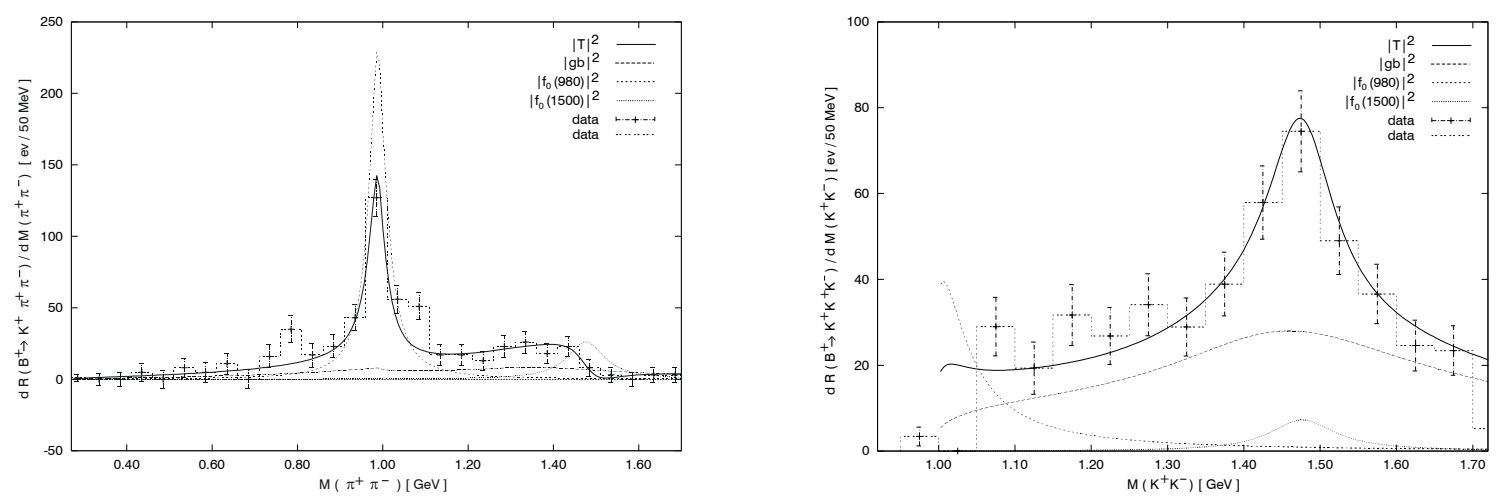

Figure 14. $\pi^{+} \pi^{-}$and $K^{+} K^{-}$mass spectra in $B$-decays (Belle [82]) in comparison with a model amplitude $|T|^{2}$ of the coherent superposition of $f_{0}(980), f_{0}(1500)$ and a glueball $(g b)$ forming the broad background. Also shown are the individual resonance terms $\left|T_{R}\right|^{2}$. The background $(g b)$ and $f_{0}(1500)$ interfere destructively in $\pi \pi$ and constructively in $K^{+} K^{-}$final states (figure from [48]).

the narrow resonances are superimposed as in (46) following Michael [99]

$$
\begin{aligned}
& T_{\pi \pi} \sim T_{g b}+c_{1} T_{f_{0}(980)} e^{2 i \delta_{g b}}+c_{2} T_{f_{0}(1500)} e^{2 i \delta_{g b}}, \\
& T_{K \bar{K}} \sim T_{g b}+c_{3} T_{f_{0}(1500)} e^{2 i \delta_{g b}}+c_{4} T_{f_{0}(1500)} e^{2 i \delta_{g b}} .
\end{aligned}
$$

Here $T_{i}$ denotes Breit-Wigner amplitudes and $\delta_{g b}$ the $S$ wave phase shift corresponding to $f_{0}(500)$ which varies between $90^{\circ}$ and $130^{\circ}$ in the considered mass range. The phase difference of the $K \bar{K}$ component, i.e. of $c_{2}$ and $c_{4}$ in (87) near $\pi$, explains the different structures around $1500 \mathrm{MeV}$ : the strong peak at $1500 \mathrm{MeV}$ in $K^{+} K^{-}$and the small enhancement at $1400 \mathrm{MeV}$ followed by a drop towards the resonance position at $1500 \mathrm{MeV}$ in the $\pi \pi$ spectrum. The interpretation in terms of subprocesses has some complexity by the contribution of different diagrams [48] and we do not discuss this further here.
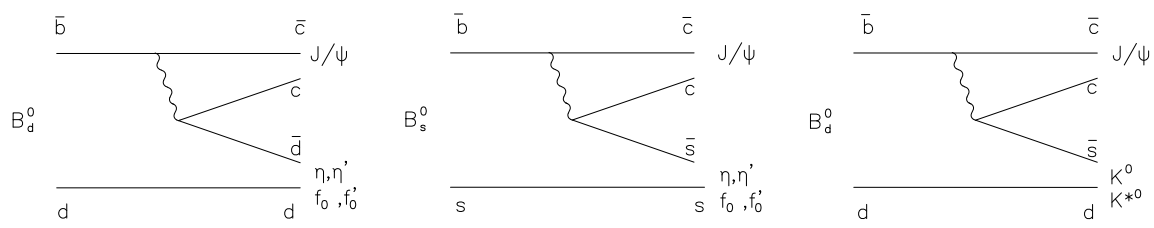

Figure 15. Leading diagrams for decays $B_{d, s}^{0} \rightarrow J / \psi \rightarrow \eta, \eta^{\prime} ; f_{0}, f_{0}^{\prime}$ and $B_{d}^{0} \rightarrow$ $J / \psi+K^{0}\left(K^{* 0}\right)$.

7.3.3. Decay $\bar{B}_{s}^{0} \rightarrow J / \psi f_{0}, f_{0} \rightarrow \pi \pi$. This process has been measured recently by Belle [163] at the $Y(5 S)$ resonance in $e^{+} e^{-}$annihilation and with higher statistics by 


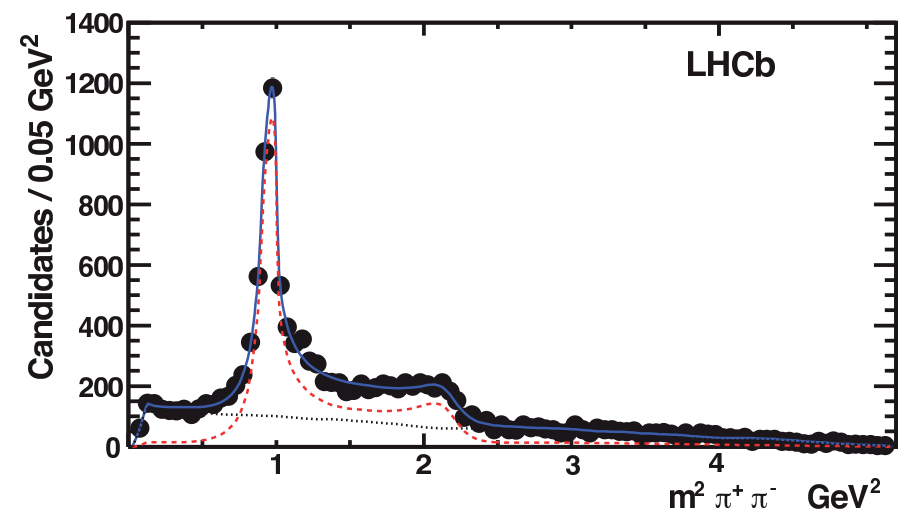

Figure 16. Mass squared spectrum $m^{2}\left(\pi^{+} \pi^{-}\right)$in $\bar{B}_{s}^{0} \rightarrow J / \psi \pi^{+} \pi^{-}$decays; the dotted and dashed lines indicate the background and signal contributions respectively, figure from [83] (LHCb Collaboration).

the LHCb Collaboration in pp collisions at $7 \mathrm{TeV}$ [83]. It proceeds dominantly through the intermediate steps

$$
B_{s} \rightarrow J / \psi+s \bar{s} ; \quad s \bar{s} \rightarrow f_{0} \rightarrow \pi \pi
$$

The dominance of the $s \bar{s}$ intermediate state has been explored in particular in connection with the $\eta-\eta^{\prime}$ mixing $[164,114,165,166]$ (see figure 15 , middle panel).

The $\pi^{+} \pi^{-}$mass spectrum has been described by both Collaborations in a model fit with contributions from $f_{0}(980)$ and $f_{0}(1370)$ above a broad background in the $\pi \pi$ $S$ wave. The LHCb Collaboration also included $f_{2}(1270)$ in the minimal fit (at a $10 \%$ level) and investigated the inclusion of small contributions from other states which led to only minor improvements. The mass spectrum shown in figure 16 shows the narrow peak from $f_{0}(980)$ and an enhancement followed by a drop near the position of the $f_{0}(1500)$ resonance $\left(m_{\pi \pi}^{2}=2.25 \mathrm{GeV}^{2}\right)$. In their preferred solution they find mass and width of the second resonance called " $f_{0}(1370)$ " as $m_{f_{0}(1370)}=1475.1 \pm 6.3 \mathrm{MeV}$, and $\Gamma_{f_{0}(1370)}=113 \pm 11 \mathrm{MeV}$. Comparing these numbers with the PDG listings we find the identification of this state with $f_{0}(1500)$ to be more appropriate: the width agrees well with $\Gamma_{f_{0}(1500)}=109 \pm 7 \mathrm{MeV}$ while it is much smaller than the PDG estimate $\Gamma_{f_{0}(1370)}=300-500 \mathrm{MeV}$. The mass is smaller by $2 \%$ in comparison with the PDG value $m_{f_{0}(1500)}=1505 \pm 6 \mathrm{MeV}$, but the mass may be affected by additional systematic effects from the interference with other contributions. Also, the " $f_{0}(1370)$ " mass is found at the upper edge of the large range suggested by PDG: $m_{f_{0}(1370)}=1200-1500 \mathrm{MeV}$. Therefore we identify the heavier resonance in $\pi \pi$ as $f_{0}(1500)$ and not as $f_{0}(1370)$ in the $\bar{B}_{s}^{0}$ decay. An additional experimental check is possible by comparison with the $K^{+} K^{-}$ channel using the respective known branching ratios; the proper inclusion of interference effects is important according to the lesson from $B \rightarrow K \bar{K} K$ above. Indeed, a signal at $1500 \mathrm{MeV}$ is observed [167] but there is an overlap with $f_{2}^{\prime}(1525)$. We also note the similarity of the mass spectra in figures 13 and 16 with peak below $1500 \mathrm{MeV}$. 
7.3.4. Mixing of $f_{0}(1500)-f_{0}(980)$ from $B_{s}$ and $B_{d}$ decays. The branching ratios for $B_{s} \rightarrow J / \psi f_{0}$ also allow tests concerning the intrinsic structure of these states. In route 2 (see table 6) they belong to the same multiplet and the coupling of $s \bar{s}$ into $f_{0}(980)$ and $f_{0}(1500)$ according to $(35)$ is approximately like $2 / \sqrt{6}$ and $-1 / \sqrt{3}$. This expectation is compared with the measurement of branching ratios in table 11.

Table 11. Fit fractions of $\bar{B}_{s}^{0} \rightarrow J / \psi f_{0}(980)$ and $J / \psi f_{0}(1500)$ for two solutions found by LHCb [83], also rescaled for unseen decays (using $\mathrm{B}\left(f_{0}(1500) \rightarrow \pi \pi\right)=0.349 \pm 0.023$ [4] and $\mathrm{B}\left(f_{0}(980) \rightarrow \pi \pi\right)=0.75 \pm 0.12$ [169]; predictions (route 2) without phase space corrections; $\left(f_{0}^{\prime} / f_{0} \simeq\left(\left(-\sin \phi_{s c}\right) / \cos \phi_{s c}\right)^{2}\right)$.

\begin{tabular}{rllll}
\hline preferred solution & $\pi^{+} \pi^{-}$fraction $\%$ & corrected $\%$ & route 2 & Phase \\
\hline $\bar{B}_{s}^{0} \rightarrow J / \psi f_{0}(980)$ & $107.1 \pm 3.5$ & $214.2 \pm 34.9$ & $\simeq(\sqrt{2 / 3})^{2}$ & 0. \\
$\rightarrow J / \psi f_{0}(1500)$ & $32.6 \pm 4.1$ & $141.6 \pm 20.1$ & $\simeq(-\sqrt{1 / 3})^{2}$ & $(241.5 \pm 6.3)^{\circ}$ \\
NonResonant & $12.84 \pm 2.32$ & $19.26 \pm 3.48$ & & $(217.0 \pm 3.7)^{\circ}$ \\
$f_{0}(1500) / f_{0}(980)$ & & $0.66 \pm 0.14$ & $0.5 \times \mathrm{PS}$ & \\
\hline Alternate solution & & & & \\
\hline $\bar{B}_{s}^{0} \rightarrow J / \psi f_{0}(980)$ & $100.8 \pm 2.9$ & $201.6 \pm 32.5$ & $\simeq(\sqrt{2 / 3})^{2}$ & 0. \\
$\rightarrow J / \psi f_{0}(1500)$ & $7.0 \pm 0.9$ & $30.4 \pm 4.4$ & $\simeq(-\sqrt{1 / 3})^{2}$ & $(181.7 \pm 8.4)^{\circ}$ \\
NonResonant & $13.8 \pm 2.3$ & $20.70 \pm 3.48$ & & $(232.2 \pm 3.7)^{\circ}$ \\
$f_{0}(1500) / f_{0}(980)$ & & $0.15 \pm 0.03$ & $0.5 \times \mathrm{PS}$ & \\
\hline
\end{tabular}

The Belle Collaboration finds the branching ratios $B\left(\bar{B}_{s}^{0} \rightarrow J / \psi f_{0}(980)\right)=$ $1.16_{-0.31}^{+0.43} \times 10^{-4}$ and $B\left(\bar{B}_{s}^{0} \rightarrow J / \psi f_{0}(1370)\right)=0.34_{-0.15}^{+0.14} \times 10^{-4}$ if we add the errors in quadrature. After corrections for unseen decay modes we find (taking the second resonance as $\left.f_{0}^{\prime} \equiv f_{0}(1500)\right)$

$r_{f_{0}^{\prime} / f_{0}}^{s}=\frac{p^{3} B\left(\bar{B}_{s}^{0} \rightarrow J / \psi f_{0}(1500)\right)}{\left(p^{\prime}\right)^{3} B\left(\bar{B}_{s}^{0} \rightarrow J / \psi f_{0}(980)\right)}, \quad r_{f_{0}^{\prime} / f_{0}}^{s}= \begin{cases}1.18 \pm 0.25) & \text { LHCb preferred solution, } \\ 0.15 \pm 0.05) & \text { LHCb alternate solution, } \\ 1.12 \pm 0.61 & \text { Belle. }\end{cases}$

with $p\left(p^{\prime}\right)$ the momenta of $f_{0}(980)\left(f_{0}(1500)\right)$ in the $B_{s}$ system. Combining the LHCb preferred solution with Belle yields

$$
r_{f_{0}^{\prime} / f_{0}}^{s}=1.17 \pm 0.23
$$

It is interesting to compare this result with the corresponding ratio for decays into $\eta$ and $\eta^{\prime}$ which should agree approximately for route 2 . This ratio has been measured recently by [168] as $R_{\eta}=B\left(B_{s} \rightarrow J / \psi \eta^{\prime}\right) / B\left(B_{s} \rightarrow J / \psi \eta\right)=0.73 \pm 0.14$ (stat.) \pm 0.02 (syst.) which leads to the phase space corrected ratio

$$
r_{\eta / \eta^{\prime}}^{s}=\frac{p_{\eta^{\prime}}^{3} B\left(B_{s} \rightarrow J / \psi \eta\right)}{p_{\eta}^{3} B\left(B_{s} \rightarrow J / \psi \eta^{\prime}\right)}=1.10 \pm 0.22
$$


This number is in a remarkable agreement indeed with the result in (90) supporting the similar flavour mixing of $f_{0}(1500)-f_{0}(980)$ and $\eta-\eta^{\prime}$ with inverse mass ordering as in (35) for route 2.

These ratios can be compared also directly with the expectations according to the quark-gluon structure of these mesons. If we allow for a gluonic component with mixing as in (18) we find (see also [166])

$$
r_{\eta / \eta^{\prime}}^{s}=\frac{\tan ^{2} \phi_{p s}}{\cos ^{2} \phi_{G}^{\eta^{\prime}}} \quad \rightarrow \quad \cos \phi_{G}^{\eta^{\prime}}=0.86 \pm 0.09 .
$$

where $\phi_{p s}=42^{\circ}$ has been used. This is easily compatible with having no gluonic component in $\eta^{\prime}$, alternatively, one can admit a contribution with $\phi_{G}^{\eta^{\prime}} \simeq\left(30.7_{-12.5}^{+8.8}\right)^{\circ}$.

Accordingly, introducing a gluonic component for $f_{0}^{\prime} \equiv f_{0}(1500)$ as

$$
\left.\left|f_{0}^{\prime}\right\rangle=\cos \phi_{G}\left|\left(\cos \phi_{s c}|n \bar{n}\rangle-\sin \phi_{s c}|s \bar{s}\rangle\right)+\sin \phi_{G}\right| g g\right\rangle
$$

we find for route 2

$$
r_{f_{0}^{\prime} / f_{0}}^{s}=\cos ^{2} \phi_{G}^{f_{0}^{\prime}} \tan ^{2} \phi_{s c} \quad \rightarrow \quad \cos \phi_{G}^{f_{0}^{\prime}}=1.87 \pm 0.32 .
$$

where we have used our result $\phi_{s c}=30^{\circ}$ in (68) and $r_{f_{0}^{\prime} / f_{0}}^{s}=1.17$ in (90). We find that this result for $\cos \phi_{G}^{f_{0}^{\prime}}$ exceeds the physical region by $2.7 \sigma$. This discrepancy may indicate a deficiency of the model with gluons mixed only into $f_{0}(1500)$ (if not of the $f_{0}-f_{0}^{\prime}$ mixing model altogether), but looking at the success of the relation $r_{\eta / \eta^{\prime}}^{s} \approx r_{f_{0}^{\prime} / f_{0}}^{s}$ one could also think of an underestimation of errors in the branching fractions of $B_{s}$ decays, of the correction factors for unseen decays or our mixing angle $\phi_{s c}$. An interesting test of the resonance analysis with $f_{0}(1500)$ would be the corresponding analysis of the decays $B_{s} \rightarrow J / \psi K \bar{K}$ where different interference phenomena appear, as we could witness already in the $B \rightarrow K+X$ decays (see figure 14 ).

The production phase of $f_{0}(1500)$ measured with respect to $f_{0}(980)$ in the $s \bar{s}$ component selected here is expected in route 2 as $\Delta \phi^{s}=180^{\circ}$ which is near the observed results in table 11. For illustration, consider our approximate representation (35): in the $n \bar{n}-s \bar{s}$ coordinate system $f_{0}(980)=(1, \sqrt{2}) / \sqrt{3}$ and $f_{0}(1500)=(\sqrt{2},-1) / \sqrt{3}$, so the relative phase for the $s \bar{s}$ component is $\Delta \phi^{s}=\pi$ and for the $n \bar{n}$ (or $d \bar{d}$ ) component it is $\Delta \phi^{d}=0$. The phase in the LHCb preferred solution is found as

$$
\Delta \phi^{s}=(241.0 \pm 6.3)^{\circ}
$$

with an additional shift by $\sim 60^{\circ}$ which could be related to a moving background phase as in the model of the type (87) (the $f_{0}(500)$ component in figure 11 moves from about $80^{\circ}$ to $140^{\circ}$ ).

The flavour mixing $f_{0}(980)-f_{0}(1500)$ can be measured also from $B_{d}$ decays, a measurement which may become feasible in the near future. In close analogy to the Cabibbo-suppressed decays $B_{d} \rightarrow J / \psi \eta^{\left({ }^{\prime}\right)}$ [164] one can study the corresponding decays into $f_{0} / f_{0}^{\prime}$. The dominant subprocesses in this case are (figure 15 , left panel)

$$
B_{d} \rightarrow J / \psi+d \bar{d}, \quad d \bar{d} \rightarrow f_{0}^{\left({ }^{\prime}\right)}, \eta^{\left({ }^{\prime}\right)} .
$$


One obtains for $B_{d}$ decays in the standard mixing scheme

$$
r_{\eta / \eta^{\prime}}^{d}=\frac{p_{\eta^{\prime}}^{3} B\left(B_{d} \rightarrow J / \psi \eta\right)}{p_{\eta}^{3} B\left(B_{d} \rightarrow J / \psi \eta^{\prime}\right)}, \quad r_{\eta / \eta^{\prime}}^{d}=\cot ^{2} \phi_{p s}
$$

In the same way, assuming an $f_{0}(980)-f_{0}(1500)$ mixing as in route 2

$$
r_{f_{0}^{\prime} / f_{0}}^{d}=\frac{p^{3} B\left(B_{d}^{0} \rightarrow J / \psi f_{0}(1500)\right)}{\left(p^{\prime}\right)^{3} B\left(B_{d}^{0} \rightarrow J / \psi f_{0}(980)\right)}, \quad r_{f_{0}^{\prime} / f_{0}}^{d}=\cot ^{2} \phi_{s c}
$$

with the same mixing angle $\phi_{s c}$. For these mixing schemes one obtains the constraints

$$
r_{\eta / \eta^{\prime}}^{d} \times r_{\eta / \eta^{\prime}}^{s}=1, \quad r_{f_{0}^{\prime} / f_{0}}^{d} \times r_{f_{0}^{\prime} / f_{0}}^{s}=1 .
$$

Also in route 2 one expects $\phi_{p s} \approx \phi_{s c}$ or

$$
r_{f_{0}^{\prime} / f_{0}}^{d} \approx r_{\eta / \eta^{\prime}}^{d}
$$

For the production phase of $f_{0}(1500)$ relative to $f_{0}(980)$ we obtain in $B_{d} \rightarrow J / \psi \pi \pi$ decays $\Delta \phi^{d}=0$, or, above a background with moving phase as in $\pi \pi$ scattering

$$
\Delta \phi^{d} \approx 60^{\circ}
$$

7.3.5. Mixing angles of isoscalars $f_{0}$ from $B_{d}$ and $B_{s}$ decays. The determination of the individual flavour mixing angles $\phi$ of isoscalars like $f_{0}(980)$ and $f_{0}(1500)$, but also of $f_{0}(500)$, i.e. the ratio of $n \bar{n}$ and $s \bar{s}$ amplitudes of these states, is possible from the ratio of $B_{s}$ and $B_{d}$ decays. As in case of $\eta, \eta^{\prime}$ [164], one can obtain the mixing angle $\phi_{s c}$ for $f_{0}(980)$ or the mixing angle $\phi_{s c}^{\prime}$ for $f_{0}(1500)$ from the ratios

$$
\begin{aligned}
& r_{f_{0}}=\frac{p_{s}^{3} \Gamma\left(B_{d} \rightarrow J / \psi f_{0}(980)\right)}{p_{d}^{3} \Gamma\left(B_{s} \rightarrow J / \psi f_{0}(980)\right)}=\frac{1}{2}\left(F_{C K M}\right)^{2} \cdot \tan ^{2} \phi_{s c} \\
& r_{f_{0}^{\prime}}=\frac{p_{s}^{3} \Gamma\left(B_{d} \rightarrow J / \psi f_{0}(1500)\right)}{p_{d}^{3} \Gamma\left(B_{s} \rightarrow J / \psi f_{0}(1500)\right)}=\frac{1}{2}\left(F_{C K M}\right)^{2} \cdot \cot ^{2} \phi_{s c}^{\prime}
\end{aligned}
$$

where $F_{C K M}=V_{c d} / V_{c s}$ is calculated for the dominant tree diagram and penguin diagram contributions and with the CKM matrix elements from PDG one finds $\left|F_{C K M}\right| \simeq 0.225$. The mixing angle $\phi_{s c}$ for $f_{0}(980)$ should agree with our determination in (68), the angle $\phi_{s c}^{\prime}$ for $f_{0}(1500)$ may be different if the two states are in different multiplets.

Under the assumption that $f_{0}(1500)$ and $K^{* 0}(1430)$ belong to the same multiplet, the mixing angle for $f_{0}(1500)$ can be determined also from the ratio

$$
r_{f_{0}^{\prime}}^{K}=\frac{p_{d}^{3} \Gamma\left(B_{s} \rightarrow J / \psi f_{0}(1500)\right)}{p_{s}^{3} \Gamma\left(B_{d} \rightarrow J / \psi K^{* 0}(1430)\right)}=\sin ^{2} \phi_{s c}^{\prime}
$$

in analogy to a corresponding ratio of $\Gamma\left(B_{s} \rightarrow J / \psi \eta\right) / \Gamma\left(B_{d} \rightarrow J / \psi K^{0}\right)$ (see figure 15). It is interesting to note, that these determinations of mixing angle between the different flavour components are independent of a possible presence of a gluonic component.

In the same way one could also study the flavour content of $f_{0}(500) / \sigma$.

We have no direct predictions from the other classification routes discussed. Some aspects of the tetra-quark model for $f_{0}(980)$ are elaborated in [114]. 


\subsection{Decay of $J / \psi \rightarrow \phi(1020) f_{0}, f_{0} \rightarrow \pi \pi, K \bar{K}$}

In the $\pi \pi$ final state of this $J / \psi$ decay the BES Collaboration [116] observed a peak around $1335 \mathrm{MeV}$ followed by a drop near $1500 \mathrm{MeV}$. In their model fits they include, besides $f_{2}(1270)$, the scalar $f_{0}(1500)$ and require $f_{0}(1370)$ as well with mass $m=1350 \pm 5$ $\mathrm{MeV}$ and width $\Gamma=265 \pm 40 \mathrm{MeV}$.

The $J / \psi$ decay is expected to proceed dominantly (according to the "singly connected diagram") through a gluonic intermediate state and, triggered by $\phi(1020)$, the $\pi \pi$ final state evolves from an intermediate $s \bar{s}$ configuration. Therefore, we expect a similar final state interaction as in the decay $B_{s} \rightarrow J / \psi \pi \pi$ (see discussion in section 7.3.3 and figure (16)) and in $\pi \pi \rightarrow K \bar{K}$ (see discussion in section 7.2.6 and figure 12). Indeed, the $\pi \pi$ spectra in these processes with an enhancement near $1400 \mathrm{MeV}$ and a drop near $1500 \mathrm{MeV}$ look all rather similar. There, evidence was only given for a single resonance interfering with some smooth background amplitude and the resonance was compatible with $f_{0}(1500)$. It is therefore important for a definite conclusion about an extra state " $f_{0}(1370)$ " to analyze the observed peak in $J / \psi \rightarrow \phi+\pi \pi$ decays with higher statistics in a bin-by-bin phase shift analysis.

In the $K^{+} K^{-}$channel the BES data [116] do not show any enhancement around $1350 \mathrm{MeV}$ and the branching fraction ratio

$$
R_{K \bar{K} / \pi \pi}=\frac{B\left(f_{0}(1370) \rightarrow K \bar{K}\right)}{B\left(f_{0}(1370) \rightarrow \pi \pi\right)}=0.08 \pm 0.08
$$

has been determined. Other measurements of this ratio listed by PDG [4] are spread over a range up to $R_{K \bar{K} / \pi \pi} \lesssim 1.0$, therefore

$$
B\left(f_{0}(1370) \rightarrow K \bar{K}\right) \lesssim 0.1 .
$$

The measurement $\Gamma(\eta \eta) / \Gamma(4 \pi)=4.7 \pm 2.0 \times 10^{-3}[170,4]$ implies this number to be an upper limit for $B\left(f_{0}(1370) \rightarrow \eta \eta\right)$. So, as an estimate

$$
B\left(f_{0}(1370) \rightarrow 2 \text { pseudoscalars }\right) \lesssim 20 \%
$$

and almost all decays should go into higher multiplicities.

\subsection{Central production in hadron hadron collisions}

Central production processes are selected by requiring two gaps in rapidity $y=\frac{1}{2} \ln \frac{E+p_{\|}}{E-p_{\|}}$, empty of particles, adjacent to the direction of the incoming hadrons, and small momentum transfers $t=\left(p_{i}-p_{f}\right)^{2}$ between the incoming and outgoing forward hadrons $a$ and $b$ (see figure 17). For such processes a description in terms of Regge theory applies. The exchange of Reggeons $I R$ or Pomerons $I P$ determines the dependence of the respective cross sections on the primary energy $\sqrt{s}$. At high energy $\sqrt{s}$ the Double Pomeron Exchange (IPIP) process dominates with an approximately constant cross section whereas subleading contributions fall like $\sigma(I P I R) \sim 1 / \sqrt{s}$ and $\sigma(I R I R) \sim 1 / s$. 


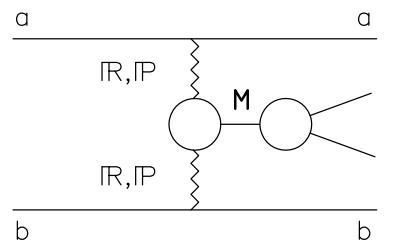

Figure 17. Central production of meson $\mathrm{M}$ in $a+b$ collisions with two rapidity gaps and exchange of Reggeons and/or Pomerons.

While the Reggeon is considered a $q \bar{q}$ object, the Pomeron is thought rather to be dominantly gluonic, more discussion follows below in section 8.1. Therefore the strength of central meson production can give a hint towards its possible gluonic component $[45,46]$. In particular, a search for scalar mesons in many different channels has been performed.

7.5.1. The $\pi \pi$ system. has been studied extensively at the ISR (Axial Field Spectrometer) [171], at the SPS collider by WA102 [172] and by GAMS [173], and now at the LHC by ALICE [174]. In figure 18 we show as an example an amplitude analysis of the $\pi^{0} \pi^{0}$ system including $S$ and $D$ waves which has been carried out using experimental moments $\left\langle Y_{L}^{M}\right\rangle$ and relations (80). Two distinct solutions appear. Solution 2 with a $D$ wave at threshold much stronger than $S$ wave is considered unphysical.

The following features of the physical solution 1 in figure (18) are noteworthy:

(i) Low mass peaks in $S$ and $D$ waves. In the $S$ wave intensity there is a strong peak at low mass $m_{\pi \pi} \sim 400 \mathrm{MeV}$ but without associated phase variation. In addition, there is also a peak in the $D_{0}^{-}$wave at $500 \mathrm{MeV}$ much below the first known resonance $f_{2}(1270)$, very different to elastic $\pi \pi$ scattering, for example. As pointed out in [175] a very similar phenomenon has actually been observed in $\gamma \gamma \rightarrow \pi^{+} \pi^{-}$(not so in $\pi^{0} \pi^{0}$ ), where the low mass $S$ and $D$ wave peaks are related to the dominant one pion exchange process at low energy with direct coupling of photons to charged pions. The pion exchange pole near the physical region of scattering at $t=m_{\pi}^{2}$ causes the enhanced forward-backward scattering generating the large $D$ wave at first. This analogy suggests the appearance of one pion exchange in the subprocess

$$
I P I P \rightarrow \pi \pi
$$

as well, which adds to the direct resonance formation as in figure 17. A Regge calculation of this exclusive process has been presented recently [176]. The presence of the broad $f_{0}(500) / \sigma$ is signaled by the interference with $f_{0}(980)$ ( $\operatorname{sharp}$ drop in $\left|S_{0}\right|^{2}$ at $m_{\pi \pi} \sim 1$ $\mathrm{GeV})$ as in $\pi \pi$ elastic scattering. At higher energies other exchange processes like $\rho$ exchange are possible [5].

(ii) There is no phase coherence. Contrary to the reaction $\pi^{-} p \rightarrow \pi^{0} \pi^{0} n$ [150] the phase differences $\varphi_{S_{0}}-\varphi_{D_{0}^{-}}$and $\varphi_{S_{0}}-\varphi_{D_{1}^{-}}$are very different. In particular, while $\left|D_{0}^{-}\right|^{2}$ and $\left|D_{1}^{-}\right|^{2}$ both show a clear signal from $f_{2}(1270)$, only $\varphi_{S_{0}}-\varphi_{D_{0}^{-}}$reflects a related phase 


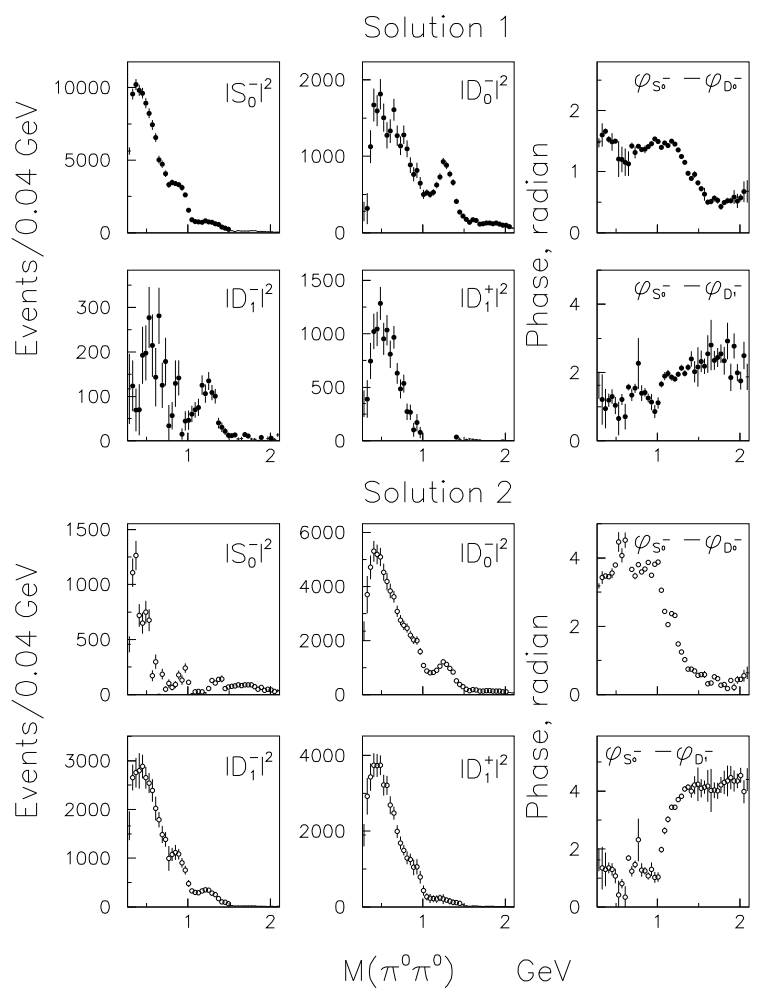

Figure 18. Partial wave amplitudes for the centrally produced $\pi^{0} \pi^{0}$ system in $p p \rightarrow p\left(\pi^{0} \pi^{0}\right) p$ at $\sqrt{s}=450 \mathrm{GeV}$, figure from [172](WA102 Collaboration).

variation from its interference with the slowly moving $S$ wave, whereas $\varphi_{S_{0}}-\varphi_{D_{1}^{-}}$is without resonance structure and so "phase coherence" is strongly violated.

Therefore, one cannot construct a model based on formulae (80) with a resonance $f_{2}(1270)$ in $D_{0}^{-}$and $D_{1}^{-}$interfering with the same $S_{0}^{-}$. The violation of phase coherence as in figure 18 is expected if $f_{2}(1270)$ couples to different partial waves $L_{\mu}^{( \pm)}$with different nucleon helicities. As an example, let the $S_{0}^{-}$wave couple only to $S_{0,++,++}^{-}$but let $f_{2}(1270)$ couple to $D_{0,++,++}^{-}$and also to $D_{1,+-,+-}^{-}$(the lower $(+)$and $(-)$refer to the nucleon helicities), then $f_{2}(1270)$ appears in both $\left|D_{0}^{-}\right|^{2},\left|D_{1}^{-}\right|^{2}$ moduli, $S_{0} \cdot D_{0}^{-}$is resonant while $S_{0} \cdot D_{1}^{-}=0$ similar to observations.

Such effects can occur if several exchange mechanisms are at work with different spin composition and "spin coherence" is violated. Then, in (80) it may be necessary to replace the single terms

$$
\begin{array}{ll}
|S|^{2} & \rightarrow|S|^{2}+|\bar{S}|^{2} ; \\
\operatorname{Re}\left(S D^{-*}\right) & \rightarrow \operatorname{Re}\left(S D^{-*}\right)+\operatorname{Re}\left(\bar{S} \bar{D}^{-*}\right)
\end{array}
$$

where the terms $S$ and $\bar{S}$ correspond to contributions with different nucleon helicities. Obviously, by addition of arbitrary incoherent contributions as in (108) one cannot in general solve for all amplitudes in an energy independent analysis, but possibly a model with selected contributions to describe all moments can be constructed. 
In a more general approach one may include a description for the transverse momentum dependences of the helicity amplitudes and in that way provide additional information for a full separation of partial wave amplitudes. Transverse momentum correlations of outgoing protons have been suggested as tool to discriminate different intrinsic structures of the centrally produced object ("glueball-filter") by Close and Kirk [177]. Different effects have been found indeed for the resonances in the $\pi \pi, K \bar{K}$ data obtained by the WA102 Collaboration [178]. Especially, the azimuthal final $p p$ correlations behave differently for $f_{2}(1270)$ and $f_{0}(1500)$ [179] which implies again that a model with "spin coherence" as incorporated in the amplitude analysis above is incomplete. The $t$-dependence of helicity amplitudes in the general framework of Regge theory and for specific models has been discussed by Kaidalov et al. [180]; and so this framework may be used for an extended analysis including helicity dependent $t$-distributions.

(iii) Contributions from states $f_{0}(1370)$ and $f_{0}(1500)$ have to be determined from a partial wave analysis, i.e. from the complex amplitudes as discussed in subsection 7.2.5. In view of the previous remarks this requires an extended analysis not relying on spin-coherence. Possibly at the higher LHC energies spin coherence is again a good approximation. In any case, a determination of the resonance fractions from $\left|S_{0}\right|^{2}$ alone without inclusion of phase movements does not yield reliable results as was demonstrated in subsection 7.2.5. For this reason the results from WA102 on $f_{0}(1370) \rightarrow \pi \pi, K \bar{K}$ $[172,178]$ are not considered here as evidence for the existence of this state.

7.5.2. Production of $f_{0}(1370)$ and $f_{0}(1500)$ in the $4 \pi$ system. The $4 \pi$ decays of the scalar mesons have been investigated extensively in central diffractive production $p p \rightarrow p(4 \pi) p($ WA102 $[181,182])$ and in $\bar{p} p \rightarrow 5 \pi^{0}$ and $\bar{p} n \rightarrow \pi^{-} 4 \pi^{0}$ annihilations (Crystal Barrel [183]). A comparative discussion is given by Klempt and Zaitsev [5]. They have collected the decay branching ratios of both resonances into different $4 \pi$ components as measured by central production and by $p \bar{p}$ annihilation experiments (see table 12)

Table 12. Decay branching ratios of isoscalar mesons into $4 \pi$ channels from central production $(\mathrm{CP})$ and $\bar{p} p / \bar{p} n$ annihilation (from [5]).

\# taken from PDG listing

\begin{tabular}{lllll}
\hline & $f_{0}(1370)$ & & $f_{0}(1500)$ & \\
& $\bar{p} p / \bar{p} n$ & $\mathrm{CP}$ & $\bar{p} p / \bar{p} n$ & $\mathrm{CP}$ \\
\hline$\Gamma_{4 \pi} / \Gamma_{\text {tot }}$ & $0.80 \pm 0.05$ & & $0.73 \pm 0.21 \#$ & $0.48 \pm 0.05$ \\
$\Gamma_{\rho \rho} / \Gamma_{4 \pi}$ & $0.26 \pm 0.07$ & $\sim 0.9$ & $0.13 \pm 0.08$ & $0.74 \pm 0.03$ \\
$\Gamma_{\sigma \sigma} / \Gamma_{4 \pi}$ & $0.51 \pm 0.09$ & $\sim 0$ & $0.26 \pm 0.07$ & $0.26 \pm 0.03$ \\
\hline
\end{tabular}

Some discrepancy for $f_{0}(1500)$ disappears, if the different decay channels included in the analysis are taken into account. In case of $f_{0}(1370)$ essential discrepancies remain. Especially, in central production there is a clear signal from $f_{0}(1500)$ in the $\sigma \sigma$ channels 
(including $4 \pi^{0}$ ) but no obvious signal from $f_{0}(1370)$ ( $\sigma$ stands here for low mass $\pi \pi S$ wave). On the other hand, in $\bar{p} p(\bar{p} n)$ there is a strong peak in the $4 \pi^{0}$ mass spectrum at $\sim 1470 \mathrm{MeV}$ but with a larger width than expected for $f_{0}(1500)$ and this effect has been related to $f_{0}(1370)$. The analysis has to take into account the contributions from different processes and decay chains in $5 \pi$ processes and combinatorial backgrounds in $5 \pi^{0}$ not present in central production. An attempt to analyze the phase motion of the $\sigma \sigma$ decay has lead to the conclusion [5] that there is only one resonant state with a motion consistent with $f_{0}(1500)$; the strong $\rho \rho$ enhancement at threshold in central production which has been related to $f_{0}(1370)$ and its complete absence in $\sigma \sigma$ in this view could be a consequence of $\rho$-Regge exchange in IPIP scattering, similar to the threshold enhancement in $\pi \pi$ which has been attributed to pion exchange as discussed before. This mechanism would explain the absence of $f_{0}(1370) \rightarrow \sigma \sigma$ in central production and similarly the absence of the $\eta \eta$ production where a suitable Regge exchange is lacking. There is also the interesting phenomenon of a dip in the $\rho \rho$ mass spectrum which moves depending on the kinematic selection for the outgoing protons [181]. For a particular selection (azimuthal angle between protons $135^{\circ}<\phi<180^{\circ}$ ) the deep sharp dip is just at the mass of $1500 \mathrm{MeV}$ which suggests a negative interference of $f_{0}(1500)$ with a broad background reminiscent of the similar phenomenon in $\pi \pi$ scattering (figure 9).

While the signature of $f_{0}(1500)$ is very clear in $(4 \pi)$, especially in $(\sigma \sigma)$, there is no compelling evidence of additional contributions which would require a new resonance at lower mass around $1370 \mathrm{MeV}$. As learned in the 2 particle channels, instead of the broad $f_{0}(1370)(\Gamma \gtrsim 300 \mathrm{MeV}$ ) there may also be a contribution from the long tail of $f_{0}(500-1000)$ with $\Gamma \sim 1000 \mathrm{MeV}$.

\subsection{Flavour structure of $f_{0}(1500)$ from its decays}

This state has been considered at first as a glueball candidate following a prediction from quenched lattice QCD. The study of decay branching ratios suggested a mixed state of $q \bar{q}$ (mainly octet) and $g g$ as discussed in route 1 above [35, 51]. A pure $q \bar{q}$ composition was found incompatible with certain decay properties [184]. On the other hand, the hint towards a negative phase between $K^{+} K^{-}$and $\pi^{+} \pi^{-}$decay amplitudes $[61,118]$ limits the contribution from a glueball component which would prefer a singlet type $q \bar{q}$ state.

A direct measurement of the mixing angle $\alpha\left(=\phi_{s c}\right.$ in route 2$)$ independent of the gluonic component is possible through the measurement of $B_{d}$ decays (see subsection 7.3.5). The negative relative sign in $D_{s}$ decay between $s \bar{s} \rightarrow f_{0}(980) \rightarrow \pi \pi$ and $s \bar{s} \rightarrow f_{0}(1500) \rightarrow \pi \pi$ (see subsection 7.3 .1 ) requires an octet type mixing angle $\alpha=\phi_{s c}$ in $(32)$ with

$$
\tan \phi_{s c}>0
$$

once the singlet type mixing for $f_{0}(980)$ is established. In the following we will investigate in more detail the consequences of the measured decay branching ratios for the constituent structure of $f_{0}(1500)$. 
The decay couplings $\gamma_{i j}^{2}$ of $f_{0}(1500)$ depend on the $q \bar{q}$ and $g g$ components through the mixing angle $\alpha$ (see (15), (32)) and the parameter $r_{G} \sim G / g$, as shown in table 3 . Now we consider ratios $R_{i}$ of branching ratios $B_{k}$ as in [35]. From the PDG fits [4] using the phase space correction $\gamma_{k}^{2}=B_{k} / q_{k}$ we derive the ratios

$$
\begin{aligned}
& R_{1}=\frac{\gamma^{2}\left[f_{0}(1500) \rightarrow \eta \eta\right]}{\gamma^{2}\left[f_{0}(1500) \rightarrow \pi \pi\right]}=0.208 \pm 0.039 \\
& R_{2}=\frac{\gamma^{2}\left[f_{0}(1500) \rightarrow \eta \eta^{\prime}\right]}{\gamma^{2}\left[f_{0}(1500) \rightarrow \pi \pi\right]}=0.21 \pm 0.09 \\
& R_{3}=\frac{\gamma^{2}\left[f_{0}(1500) \rightarrow K \bar{K}\right]}{\gamma^{2}\left[f_{0}(1500) \rightarrow \pi \pi\right]}=0.321 \pm 0.034
\end{aligned}
$$

(using also $q_{\eta \eta^{\prime}}=195 \mathrm{MeV}$ for $R_{2}$ [35]). For a given experimental ratio $R_{i}$ we can insert in (110)-(112) the results for $\gamma_{i j}^{2}$ from table 3 . Then, for each value of the mixing angle $\alpha$ there are two corresponding solutions for the glue contribution $r_{G i}^{ \pm}$. Solving for $r_{G i}^{ \pm}$ we find (with $\phi \equiv \phi_{p s}$ and $\rho=R=1$ )

$$
\begin{aligned}
& r_{G 1}^{ \pm}\left(\sqrt{3 R_{1}} \mp 1\right)=-\sqrt{3 R_{1}} \cos \alpha \pm\left(\cos ^{2} \phi \cos \alpha-\sqrt{2} \sin ^{2} \phi \sin \alpha\right) \\
& r_{G 2}^{ \pm} \sqrt{3 R_{2}}=-\sqrt{3 R_{2}} \cos \alpha \pm \sqrt{2} \sin \phi \cos \phi(\cos \alpha+\sqrt{2} \sin \alpha) \\
& r_{G 3}^{ \pm}\left(\sqrt{3 R_{3}} \mp 2\right)=-\sqrt{3 R_{3}} \cos \alpha \pm(\cos \alpha-\sqrt{2} \sin \alpha)
\end{aligned}
$$

In figure 19 (left panel) these solutions $r_{G i}^{ \pm}$for every $R_{i}$ are shown for the given $\alpha$. There are two special points for $\tan \alpha=-1 / \sqrt{2}$ where all solutions coincide in a trivial way if the numerator $N_{i}$ and the denominator $D_{i}$ for $R_{i} D_{i}=N_{i}$ vanish:

$$
\alpha_{1} \approx-35^{\circ}, \alpha_{2} \approx 145^{\circ} ; \text { with } r_{G 1} \approx-0.82, r_{G 2} \approx 0.82
$$

Otherwise, there is no crossing of the solutions from the three ratios $R_{i}$ expected for an exact solution for $r_{G}$, but rather some pairs of ratios come close to each other $\left(r_{G 2}^{+}, r_{G 3}^{+}\right)$ and $\left(r_{G 1}^{-}, r_{G 3}^{-}\right)$.

In the right panel of figure 19 we show, how the $\chi^{2}$ varies with the glue component $r_{G}$ for a mixing angle $30^{\circ}$; these two parameters determine the ratios $R_{i}$ according to table 3. Also shown are the contributions to $\chi^{2}$ from $R_{i}$ separately which vanish at the exact solutions for $r_{G i}^{ \pm}$seen in figure 19 (left). There is a $\chi^{2}$ minimum

$$
\text { for } \alpha=30^{\circ}: \quad r_{G}=+0.7 \pm 0.1 \text {. }
$$

For this solution there is a good agreement with $R_{2}, R_{3}$ but a discrepancy with the $\eta \eta$ channel $\left(R_{1}\right)$ by $\gtrsim 2 \sigma$. There is another solution for $r_{G} \sim-0.4$ but with an unacceptable $\chi^{2}>30$. The $\chi^{2}$ minimum is found to be practically independent of $\alpha$ if $r_{G}$ moves along the solutions $\left(r_{G 2}^{+}, r_{G 3}^{+}\right)$. On that path there is also the solution $\left(\alpha=0^{\circ}, r_{G} \approx 0\right)$ for $f_{0}(1500)$ without glue. Therefore, it needs further information to determine the mixing angle $\alpha$ uniquely. Some information comes from the $2 \gamma$ decay rate (see, for example [184]) which needs some model assumptions (see next subsection). If $f_{0}(980)$ and $f_{0}(1500)$ belong to the same multiplet as in route 2 then there are further relations as well as discussed above. A direct determination of the mixing angle is possible by 

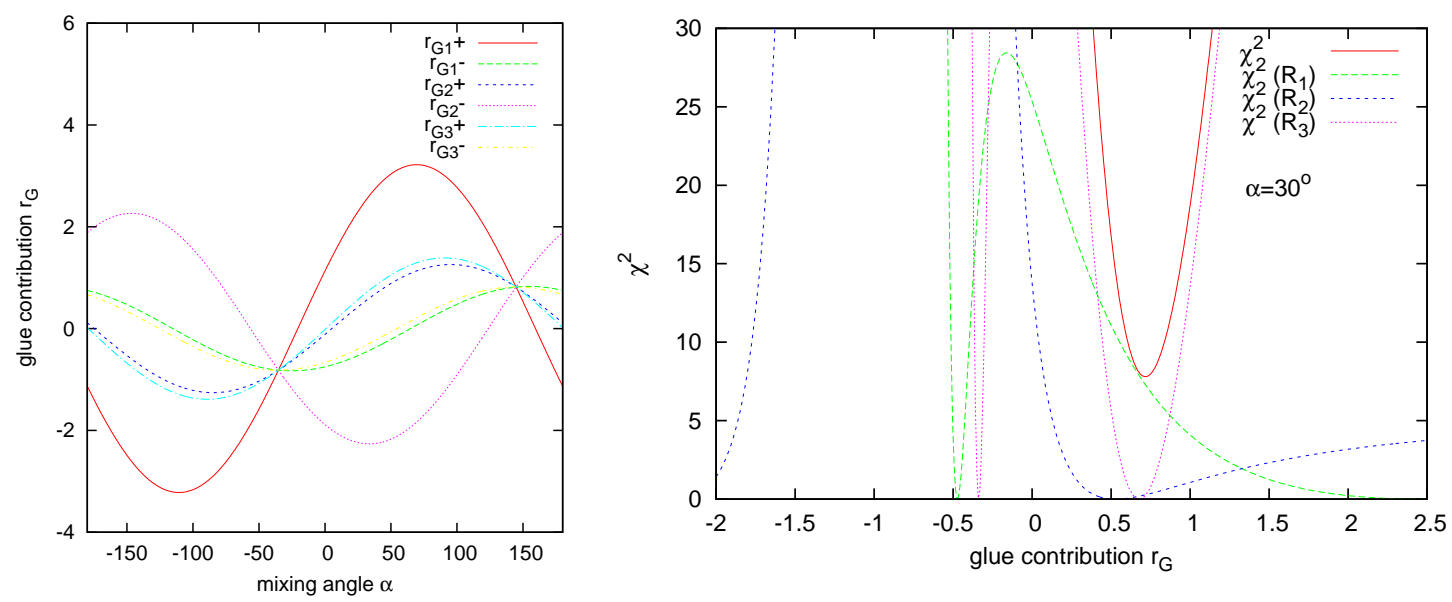

Figure 19. Glue contribution $r_{G}$ in the $f_{0}(1500)$ state as function of mixing angle $\alpha=\phi_{s c}$ for fixed ratios $R_{i}$ of branching ratios as in (110)-(112) (left); $\chi^{2}$ vs. $r_{G}$ for observed ratios $R_{i}$ and full $\chi^{2}$ at mixing angle $\alpha=30^{\circ}$ (right).

comparing $B_{d}$ and $B_{s}$ decays as in (103). Further information is available from the interference with other states like $f_{0}(980)$ or $f_{0}(500-1000)$ in different channels.

According to Anisovich [185] the decay coupling $g_{0}$ of the $q \bar{q}$ state into mesons and the coupling $G_{0}$ for the gluonium decay occur in the same leading order of a $1 / N_{c}$ expansion. Then the couplings in (24) are of the same order

$$
g_{0} \approx G_{0}
$$

For the allowed solution of $r_{G}$ following $r_{G 2}^{+}, r_{G 3}^{+}$we deduce from the figure 19 the bounds $-1 \lesssim r_{G} \lesssim 1$ and with condition (109) from the $f_{0}(980)-f_{0}(1500)$ interference

$$
0 \lesssim r_{G} \lesssim 1
$$

so for $f_{0}(1500)$ the gluonium contribution $\simeq G$ does not exceed the quarkonium contribution $\simeq g$. As an example, if we assume the mixing $\alpha=\phi_{s c} \approx 30^{\circ}$ as in route $2\left(\phi_{s c} \approx \phi_{p s}\right)$ then we find the glue component in (117)

$$
G / g \approx \tan \phi_{G} \approx 0.7 ; \quad \phi_{G} \approx 35^{\circ}
$$

The alternative solutions $r_{G 1}^{-}, r_{G 3}^{-}$have typically opposite sign and are disfavoured by $\chi^{2}$.

\subsection{Production in $\gamma \gamma$ scattering}

The Belle Collaboration [94] has measured the angular distribution of the process

$$
\gamma \gamma \rightarrow \pi^{0} \pi^{0}
$$

with high statistics. A partial wave analysis of the $\pi^{0} \pi^{0}$ system in the cms energy range $0.6 \mathrm{GeV} \leq W_{\gamma \gamma} \leq 1.6 \mathrm{GeV}$ has been carried out with $S, D_{0}$ and $D_{2}$ waves 
included. As the photons are unpolarized some ambiguities remain. A parameterization in terms of resonances and backgrounds yields parameters for $f_{2}(1270)$ and in the $S$ wave for $f_{0}(980)$ and an additional resonance $Y$, either $f_{0}(1370)$ or $f_{0}(1500)$. They obtain the mass $m=1470_{-7-255}^{+6+72} \mathrm{MeV}$ and the total width $\Gamma=90_{-1-22}^{+2+50} \mathrm{MeV}$ and partial

width $\Gamma_{\gamma \gamma} B\left(f_{0}(Y) \rightarrow \pi^{0} \pi^{0}\right)=11_{-2-7}^{+4+603} \mathrm{eV}$. The large systematic errors are related to uncertainties in the coupling of helicity $\mu=0$ of $f_{2}(1270)$ and the $K \bar{K} / \pi \pi$ branching ratio of $f_{0}(980)$. Mass and width are nicely compatible with PDG values for $f_{0}(1500)$ while for the alternative $f_{0}(1370)$ the width is smaller than the PDG estimate $300-500$ $\mathrm{MeV}$.

With the identification of the extra state as $f_{0}(1500)$ we can correct the above radiative width for other hadronic decay modes and obtain

$$
f_{0}(1500): \quad \Gamma_{\gamma \gamma} / \Gamma_{\text {tot }}=95_{-17-60}^{+34+519} \mathrm{eV}, \quad \Gamma_{\gamma \gamma}^{f_{0}(1500)} / \Gamma_{\gamma \gamma}^{a_{0}(980)}=0.38_{-0.07-0.24}^{+0.14+2.08}
$$

We may also compare with constituent models. Assuming a mixing as in $\eta$ according to route 2 with $f_{0}(1500) \sim(u \bar{u}+d \bar{d}-s \bar{s}) / \sqrt{3}$ we obtain the ratio, following [61]

$$
\begin{aligned}
\Gamma_{\gamma \gamma}^{f_{0}(1500)} / \Gamma_{\gamma \gamma}^{a_{0}(980)} & =\frac{32}{27} \times\left(m_{f_{0}} / m_{a_{0}}\right)^{p} \mathrm{eV} \\
& =0.53 / 1.8 / 4.0 \mathrm{eV} \text { for } p=-3 / 1 / 3
\end{aligned}
$$

There is some uncertainty in the extrapolation in mass from the $a_{0}(980)$ to $f_{0}(1500)$. In Born approximation which is good for light pseudoscalars the power in (122) is $p=3$; such a power is also consistent with the radiative widths of the lightest tensor meson nonet if the mixing angle $\alpha_{T}=81^{\circ}$ is used [3]. Taking only phase space with $p=1$ or a form factor with $p=-3$ we find the lower values in (122). Given the large upper systematic experimental error there is no real contradiction with the pure quark composition models, on the other hand, there is enough room for gluonic contributions which would lower the $q \bar{q}$ predictions accordingly.

\section{Gluon rich processes in comparison}

So far, the different routes for classification of scalar mesons have not yet led to a generally accepted identification of a light glueball. In route 1 and 3 there is the need for $f_{0}(1370)$ where a clear evidence is missing: there is no established 2-body decay and their total decay rates are strongly limited (10-20\%); the decay rates into higher multiplicities are controversial. A good argument why 2-body decays should be strongly suppressed in comparison to $f_{0}(1500)$, for example, is lacking as well. New experiments, for example COMPASS [186] could reach higher sensitivities in such studies.

In route 2 , the asymmetry between $\sigma$ and $\kappa$ is a bit puzzling, although not fatal; $f_{0}(500)$ may not be purely gluonic and the mixing properties of isoscalars have to be better understood, possibly from $B_{d}, B_{s}$ decays.

The existence of glueballs as a basic prediction of QCD is now dependent on the existence of a supernumerous state in the classification which is found controversial. Therefore there was the hope to get an additional signal from the enhanced production 
of glueballs in "gluon enriched" reactions. Looking over the discussions of the previous sections, there is not such a really striking predominance of a particular isoscalar state in the gluon rich processes (i)-(iii) listed in subsection (3.2.1).

The reason could be that the relevant energies are of the order of the glueball mass in question itself in the range 1-2 GeV which may be too low for a gluon to appear as a well distinguished object. Rather, because of the running strong coupling the presence of intermediate $q \bar{q}$ components at low energies may be important as well. As an example, we consider the double Pomeron process (ii) and then we investigate processes where the presence of a gluon as primary agent is better established.

\subsection{The Pomeron as an example for mixed gluon and quark components}

At LHC energies of $7 \mathrm{TeV}$ the double Pomeron process should dominate over Regge exchange processes for sufficiently large rapidity gaps. The Pomeron has isospin $I=0$. If it is primarily a gluonic object we expect the emergence of gluonic mesons in such processes. A first result from the ALICE Collaboration with gap selections is shown in figure 20 . While the $\pi \pi$ spectrum without gap selection shows the $\rho$ meson as dominant

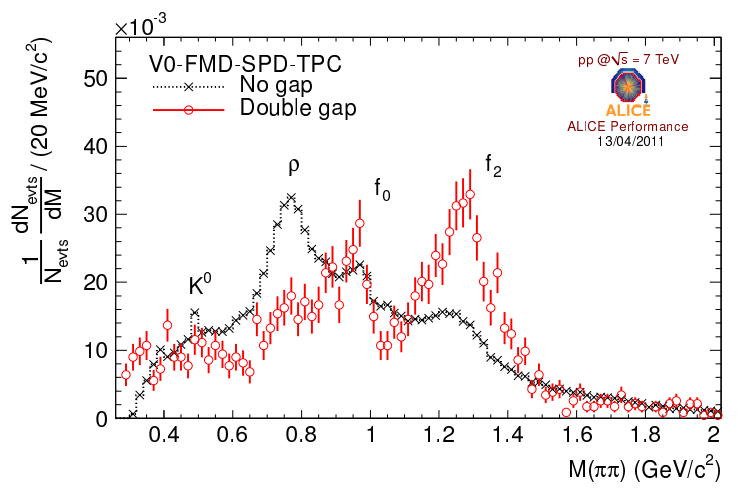

Figure 20. Central production of pion pairs at the LHC at $7 \mathrm{TeV}$ with and without gaps in pseudorapidity $-3.7<\eta<-0.9$ and $0.9<\eta<5.1$ (not corrected for acceptance), figure from [174] (ALICE Collaboration).

structure, this state disappears if double gap events are selected. This is expected for the double Pomeron exchange process which cannot yield a final $\rho$ meson with isospin $I=1$. Now, for the double gap events, the previously small structures related to $f_{0}(980)$ and $f_{2}(1270)$ become dominant. Certainly, the $f_{2}(1270)$ is a "classical" $q \bar{q}$ state as is nicely confirmed by the 2-photon decay rates according to the quark model (see, for example, [3]). The $f_{0}(980)$ is not typically considered as gluonic, though a small gluonic component cannot be excluded (see section (6)). Therefore, this result confirms that the double Pomeron process selects isoscalar states, but there is no evidence for the selection of gluonic states in particular. Such a behaviour may be related to a Pomeron, which is constructed from mixed glueball and $q \bar{q}$ trajectories as it is realized within a non-perturbative QCD approach [187]. 
In this context, it is interesting to recall the results from the studies of deep inelastic scattering at the HERA ep collider by the H1 [188] and ZEUS [189] Collaborations on the partonic structure of the Pomeron as obtained from events with a rapidity gap adjacent to the target proton. At large momentum fraction $z$ of partons inside the Pomeron the valence component is expected to dominate. It is found, that for $z \gtrsim 0.8$ (for all scales presented with $Q^{2} \gtrsim 6 \mathrm{GeV}^{2}$ ) the flavour singlet quark density dominates over the density of gluons. In a perturbative QCD treatment such dominant quark densities are initiated by Regge-like contributions and evolve according to the QCD equations for the quark and gluon densities [190]. Only in a global view, the gluonic component dominates: the integrated gluon density is much bigger than the quark density and the gluons carry a larger fraction of the total momentum as compared to the quarks.

\subsection{Processes with identified gluons}

In the gluon-rich processes (i)-(iv) the existence of gluons as intermediate partons is required by theoretical arguments but at the low energy we cannot be sure about the competing role of quark processes. We are therefore looking after processes involving higher gluon energies where the primary identity of gluons is better known.

This is the case in the high energy production of quarks and gluons which evolve into hadronic jets. The identity of quark jets became obvious in $e^{+} e^{-}$annihilations above around $\sqrt{s} \sim 7 \mathrm{GeV}$ at SPEAR [191] whereas gluon jets have been identified around $30 \mathrm{GeV}$ at PETRA [192]. With increasing jet energies, the quark and gluon jets can be better distinguished (see, for example [193], for a review). At LHC the knowledge of parton distribution functions allows the calculation of rates for quark and gluon jets and the selection of jets with a high purity ( $\gtrsim 80 \%)$ of either quark or gluon jets [194]. Then the fragmentation into particles of high momentum fraction $z$ may yield information about gluonic mesons (see below in section 9).

Of special interest are exclusive 2-body channels $(z \sim 1)$ which can be more easily identified also at lower energy and may carry a primary quark or gluon. An example is

the weak ("penguin") decay of the heavy $b$ quark (iv) $b \rightarrow s g$ which may contribute to $B \rightarrow K f_{0} \rightarrow K \pi \pi$. This processes have been studied in [48]. There are interesting tests for the members of the scalar meson multiplets but the isolation of the gluonic process has not been achieved so far.

A promising study concerns the $P$ wave charmonium (or bottomium) $\chi_{c}$ (or $\chi_{b}$ ) decays (v) which proceed through $\chi \rightarrow g g$ with primary gluons (or other $C=+1$ heavy quarkonia) to which we turn in section 10.

\section{Leading systems in gluon jets}

A promising possibility to identify gluonic mesons is provided by the comparative study of leading particle systems in quark and gluon jets (see [195] for a recent discussion). According to the well known concept of quark fragmentation the leading particles at 
large momentum fraction "Feynman $x$ " are those which carry the primary quark of the jet as a valence quark, see figure 21. For example, leading particles in a $u$ quark jet are
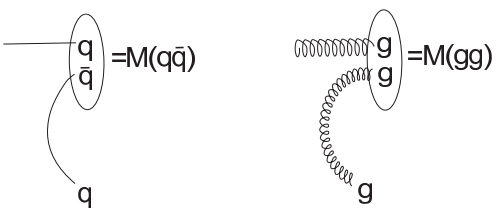

Figure 21. Leading $q \bar{q}$ and gluonic particles in quark and gluon jets.

$\pi^{+}(u \bar{d})$ or $\pi^{0}(\{u \bar{u}+d \bar{d}\} / \sqrt{2})$ with half strength whereas $\pi^{-}$or $K^{-}$without valence $u$ quark are suppressed at large $x$. In analogy, one can consider the fragmentation of a gluon and hypothesize that the leading particle has a gluonic valence component.

Models of this kind with leading glueballs in jets, and also with leading isoscalars like $\eta, \eta^{\prime}, \omega$ at large $x$ have been suggested already long ago [196], $x$-distributions have been considered in [197]. The model for isoscalar $q \bar{q}$ meson production has been studied at LEP with quark and gluon jets [198], but no clear experimental support for the model has been established [195]. This means that the isoscalars $\eta$ and $\eta^{\prime}$ behave like other $q \bar{q}$ mesons in fragmentation.

A critical test for the presence of gluonic objects in the leading system of a jet

is the measurement of charge and mass of the leading cluster $Q_{\text {leading }}$ and $M_{\text {leading }}$ beyond a rapidity gap. Their distribution is expected to reflect the mechanism for colour neutralization [199]. In a non-perturbative approach an initial pair of $q \bar{q}$ with colour triplet charges creates another $q \bar{q}$ pair to neutralism the colour charges beyond the confinement radius $R_{c}$. For an initial $g g$ pair the colour may be neutralized either by another $g g$ pair ("colour octet neutralization") or by creation of two $q \bar{q}$ pairs ("colour triplet neutralization"), see figure 22. In a high energy jet one expects the charge distribution of the leading cluster to approach with increasing rapidity gap a limiting behaviour with charge $Q_{\text {leading }}=0$ for colour octet $(g g)$ and charge $Q_{\text {leading }}=0, \pm 1$ for color triplet $(q \bar{q} g)$ neutralization according to the minimal configuration of partons beyond the gap. The mass distribution of the leading system with $Q_{\text {leading }}=0$ should signal the presence of glueballs if any.

\subsection{Rate of neutral clusters at LEP above Monte Carlo expectations}

The distributions of charge and mass for the leading clusters in quark and gluon jets have been measured in $e^{+} e^{-}$annihilation at LEP by the DELPHI [200], OPAL [201] and ALEPH [202] Collaborations. The results by DELPHI on the distribution of the leading charge beyond a rapidity interval $\Delta y=1.5$ are presented in figure 23 . For the most energetic jet in a 3-jet event ("jet1", in $90 \%$ of cases a quark jet) the distribution of the leading charge (sum of charges "SQ") follows closely the Monte Carlo expectation (JETSET [203]). This numerical calculation is based on the QCD approach to parton cascade evolution and string hadronization after tuning free parameters by experimental 
(a)

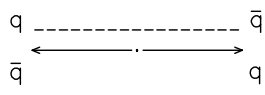

(b)

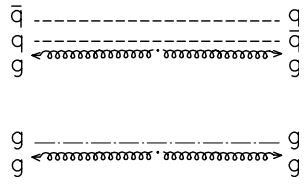

Figure 22. Colour neutralization in quark and gluon jets (figure from [199]).

data. On the other hand, for the jet of lowest energy ("jet3", a gluon jet with purity $70 \%$ ) there is a significant excess of events with $Q_{\text {leading }}=0$, clearly visible in the lower figures for the difference "data-MC". This excess is also shown in [200] to be built up by increasing the rapidity gap $\Delta y$.
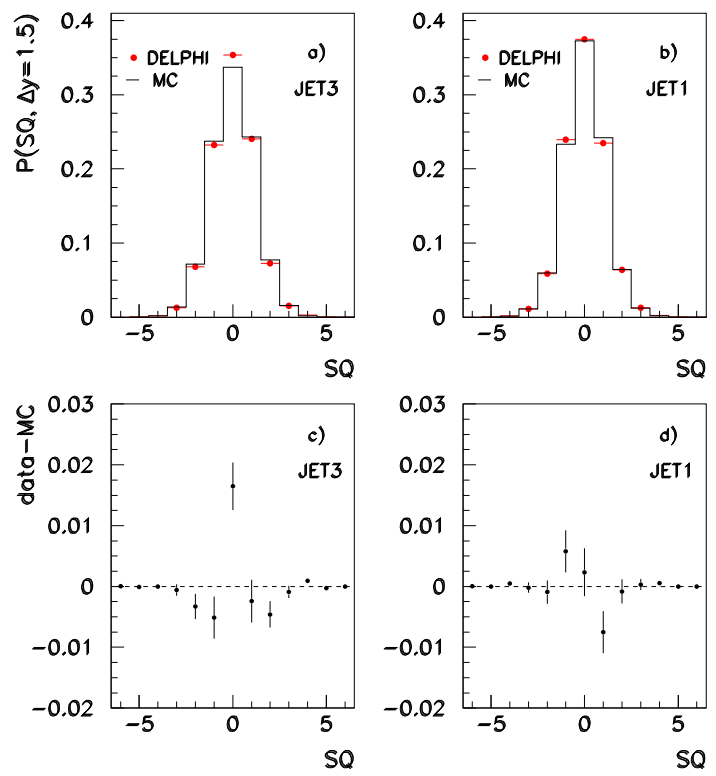

Figure 23. Distribution of the sum of charges "SQ" (" leading charge") beyond a rapidity gap of $\Delta y=1.5$ in gluon dominated (jet3) and quark dominated (jet1) jets in comparison with Monte Carlo (JETSET); analysis by DELPHI, showing the surplus of $S Q=0$ events in gluon jets (figure from [200]).

In the analysis by ALEPH [202] gluon jets of high purity have been selected from the 3 -jet events $b \bar{b} g$ with identified $b$-quark jets. The distribution of charge has been studied in gluon jets without gap and in gluon jets with a rapidity gap. As can be observed in figure 24, the charge distribution in the gluon jet without gap follows the MC calculation (JETSET) whereas there is a large excess by about $40 \%$ in the jet with a rapidity gap. Models with non-standard colour connections along the parton cascade fail to reproduce the data as well.

Finally, the distribution of masses of the leading cluster in figure 25, left side, shows again a good agreement with the predictions from the JETSET Monte Carlo for quark jets (right column), whereas for gluon jets the excess of events with leading charge 

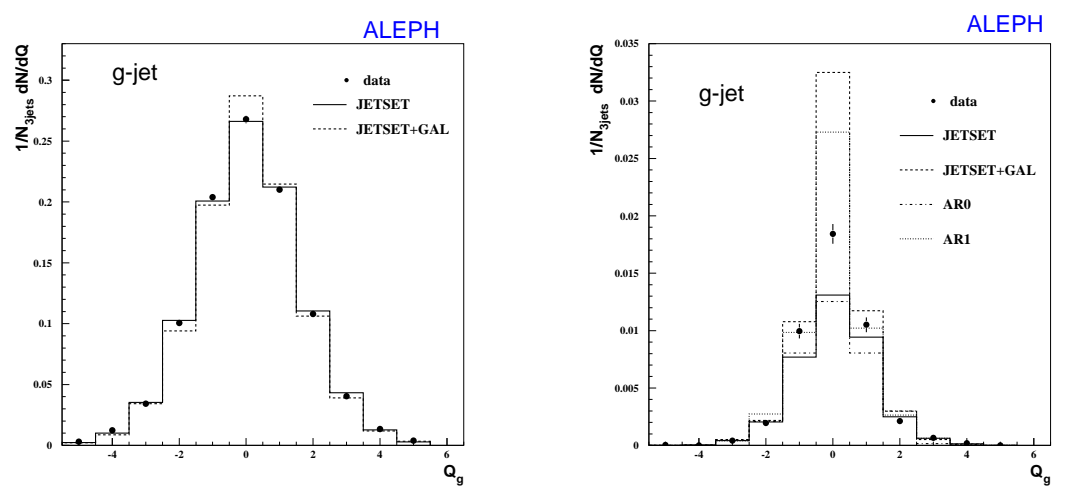

Figure 24. Left: distribution of the leading charge $Q_{\text {leading }}$ in a gluon jet of high purity without gap; right: the same for gluon jets with rapidity gap $\Delta y=1.5$ showing the surplus of events for $Q_{\text {leading }}=0$ over the JETSET Monte Carlo; other models (GAL, AR) with "colour reconnection", figure from [202] (ALEPH Collaboration).

$Q_{\text {leading }}=0$ is observed at small masses, mainly for $M \lesssim 2 \mathrm{GeV}$. An excess of events at small masses is also observed by OPAL (figure 25, right hand side). The mass spectra for selected charges with $Q_{\text {leading }}=0$ don't indicate striking resonant structures as expected for narrow glueballs, a small enhancement around $1500 \mathrm{MeV}$ in the $(+-+-)$ spectrum is indicated while for $(+-)$ the mass distribution is smooth. Interestingly, there is no $\rho$ signal which the models seems to predict; this could indicate a special feature of gluon jets which may not just be built from a long $q \bar{q}$ colour triplet string.

While the study of spectroscopy did not provide a definitive evidence for a gluonic component in the spectrum, the inclusive production of the leading clusters in a gluon jet appears to be different from what is expected within a model which reproduces otherwise the main feature of the multiparticle final states in $e^{+} e^{-}$annihilations. The lack of better signals from resonances may indicate that in the selection procedure for rapidity gaps particles from a resonance have been lost. Also the rapidity gap with $\Delta y \simeq 1.5$ may be too small for significant results.

\subsection{Proposal for further measurements at the $L H C$}

There are some advantages to follow this line of studies at $p p$ colliders, especially at the LHC [195]:

- there is access to jets of much higher energies in comparison to LEP, therefore larger rapidity gaps are available with better separation of the leading cluster;

- quark and gluon jets can be analyzed at comparable energies in the same experiment;

- the gluon jet sample could be much larger than before.

At $p p$ colliders quark and gluon jets can be produced in $2 \rightarrow 2$ processes of leading order: quark jets in $p p \rightarrow \gamma+j e t+X$ with subprocess $q g \rightarrow \gamma q$ dominant at the lower 

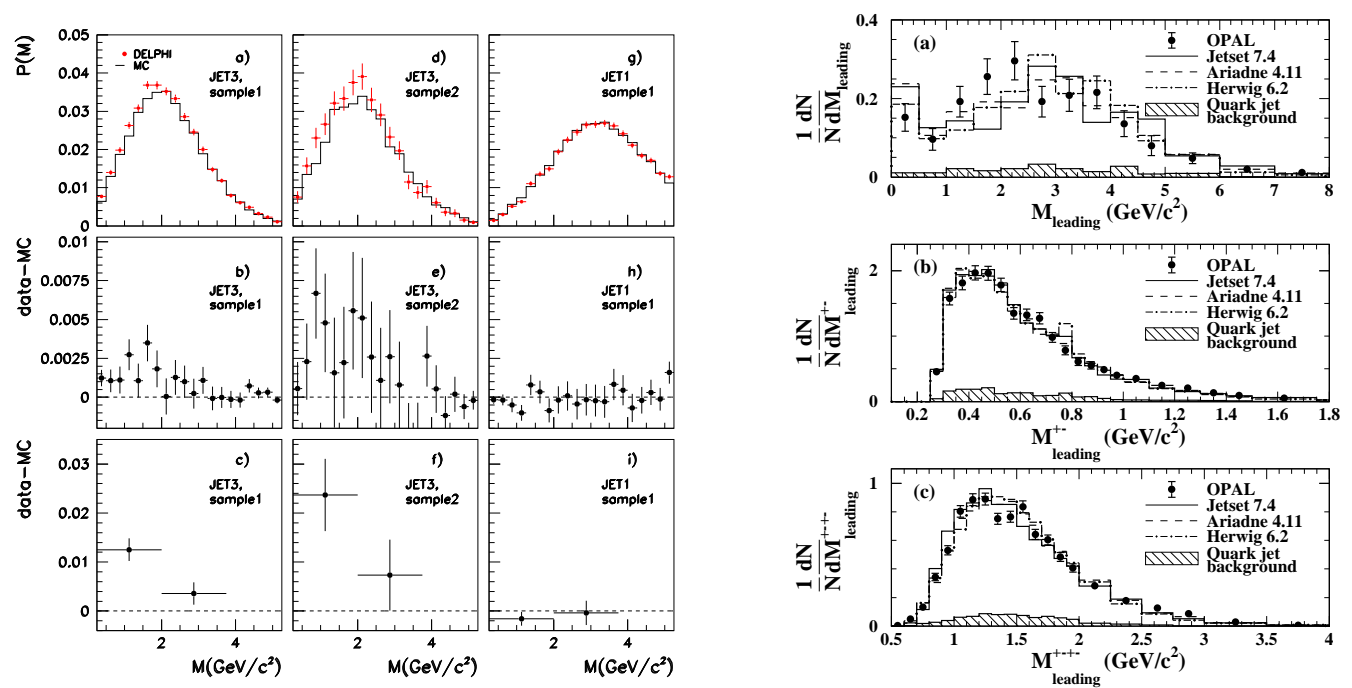

Figure 25. Mass density distributions for the leading cluster $M_{\text {leading }}$ of charged and neutral particles; left: DELPHI results for gluon jets (JET3 of lowest energy in 3 jet events (sample 1) and in $b \bar{b} g$ events (sample 2)) and for quark jets (JET1of highest energy), all with charge $Q_{\text {leading }}=0$ (figure from [200]); right: OPAL results for all mass combinations and for clusters with charges $(+-)$ and $(+-+-)$, figure from [201].

Table 13. Purities (in\%) of quark and gluon jets at Tevatron [204] and at LHC [194], taken from [195].

\begin{tabular}{lccccc}
\hline & & $p_{T}$ & $x_{T}$ & $\mathrm{~g}$ in di-jet & $\mathrm{q}$ in $\gamma+$ jet \\
\hline Tevatron & $1.8 \mathrm{TeV}$ & 50 & 0.056 & $60 \%$ & $75 \%$ \\
LHC & $7 \mathrm{TeV}$ & 200 & 0.057 & $60 \%$ & $80 \%$ \\
& & 50 & 0.014 & $75 \%$ & $90 \%$ \\
& & 800 & 0.229 & $25 \%$ & $75 \%$ \\
\hline
\end{tabular}

$p_{T}$ and gluon jets in di-jet events also at the smaller $p_{T}$. The rates can be calculated from parton distribution functions and parton-parton cross sections, the purities depend on primary energy and $p_{T}$, for some sample calculations see table 13. For quark jets a purity of $80 \%$ seems sufficient for the study of leading systems (quarks fragment harder than gluons), for gluons a higher purity of $>90 \%$ is desirable. This can be achieved by triggering on large total transverse energy and the selection of 3-jet events. Similar to the case of $e^{+} e^{-} \rightarrow 3$ jets the lowest momentum jet is most likely a gluon jet from QCD Bremsstrahlung ( $q q g$, qgg or $g g g$ ). Estimates suggest that purities of $95 \%$ for gluon jets are within reach [195]. Studies at LHC could be important in two directions:

9.2.1. Leading clusters with larger rapidity gaps. The energies of identified quark and gluon jets at the LHC could be larger by an order of magnitude as compared to LEP (typically $<25 \mathrm{GeV}$ ) and the rapidity gaps could extend up to $\Delta y \sim 4$. Then, the leading charges are expected closer to their asymptotic values $Q_{\text {leading }}=0, \pm 1$ with a 
better sensitivity to an extra component with $Q_{\text {leading }}=0$.

9.2.2. Identifying gluonic mesons through $[q \bar{q}]$-reference $x$-spectra. The observed mass spectra of the leading cluster beyond the rapidity gap did not show prominent resonance structures. As the rapidity gap selection cuts into the angular distribution of the cluster decay particles the resonance signal may be reduced. In an alternative approach, the mass spectra of particle clusters $M(\pi \pi), M(K \bar{K}), M(4 \pi) \ldots$ are measured at a given total energy fraction $x$ of the cluster and the $x$-distribution of resonances is determined. Such resonance $x$-spectra have been determined in the past [205], but not separately for quark and gluon jets.

The identification of gluonic mesons $g b$ by the classification as "leading" and "nonleading" appears feasible by the comparison of the $x$-spectra $D^{g b}(x)$ with the spectra of reference $[q \bar{q}]$ mesons of similar mass $D^{[q \bar{q}]}(x)$. In comparison with the "leading" spectrum like $u \rightarrow \pi^{+}$a gluonic meson should be suppressed

$$
D_{u}^{g b} \ll D_{u}^{\pi^{+}} \text {for large } x .
$$

Similarly, a gluonic resonance $f_{0}(g b)$ should be suppressed in a quark jet as compared to a reference $[q \bar{q}]$ resonance and, vice versa, a $[q \bar{q}]$ meson should be suppressed in a gluon jet, as summarized in table 14 (for tetraquark mesons we assume here a steeper fragmentation as for $[q \bar{q}]$ mesons according to the counting rules [206]). As an example,

Table 14. Mesons are leading or suppressed in fragmentation $x$-spectra depending on whether they carry the jet initiating parton $(q$ or $g)$ as a valence constituent; gluonic mesons are detected by comparison to reference $q \bar{q}$ mesons

\begin{tabular}{lllll}
\hline meson & & quark jet & $\begin{array}{l}\text { gluon jet } \\
\text { triplet neutr. }\end{array}$ & octet neutr. \\
\hline$[q \bar{q}]:$ & $f_{0}\left\{r e f: \rho, f_{2}\right\}$ & leading & suppressed & suppressed \\
$g b:$ & $f_{0}\left\{r e f: \rho, f_{2}\right\}$ & suppressed & suppressed & leading \\
{$[4 q]:$} & $\sigma, f_{0}(980)(?)$ & suppressed & suppressed & suppressed \\
\hline
\end{tabular}

for a gluonic scalar meson $f_{0}(g b)$ decaying to $\pi \pi$ (candidates $\left.f_{0}(500), f_{0}(980), f_{0} 1500\right)$ ) we can take $\rho(770)$ and $f_{2}(1270)$ as reference $[q \bar{q}]$ states. Then,

$$
\text { at large } x: \quad D_{q}^{f_{0}(g b)}(x) \ll D_{q}^{\rho, f_{2}}(x) ; \quad D_{g}^{f_{0}(g b)} \gg D_{g}^{\rho, f_{2}}(x) .
$$

As found by the OPAL Collaboration [207] the $x$-distribution of $f_{0}(980)$ and $f_{2}(1270)$ almost coincide, then there is no evidence so far for any constituent structure beyond $q \bar{q}$ of $f_{0}(980)$ from this study; a similar measurement of the corresponding $x$ spectra in gluon jets could reveal a gluonic admixture of $f_{0}(980)$ if there appeared a hard component at large $x$. In this way, also mixed $g b-q \bar{q}$ states could be recognized.

Gluonic components could appear in the spectra of $(\pi \pi)^{0}$ from $f_{0}(500) / \sigma, f_{0}(980)$, $f_{0}(1500)$; in $(4 \pi)^{0}$ from $f_{0}(1370)(?), f_{0}(1500)$ and in $(K \bar{K})^{0}$ from $f_{0}(980), f_{0}(1500)$ and $f_{0}(1710)$. As an example, if $f_{0}(1500)$ has a large glueball component, it should fragment harder than $\rho$ or $f_{2}$ in a gluon jet and softer in a quark jet. 


\section{0. $[q \bar{q}]$ multiplets and glueballs from symmetry relations: first results from $\chi_{c}$ decays}

In this section we investigate the exclusive production of hadronic states $H_{1}, H_{2}$ in decays of heavy Quarkonia with $C=+1$ through the intermediate process

$$
[Q \bar{Q}] \rightarrow g g \rightarrow H_{1} H_{2}
$$

in particular in decays of $\chi_{c}$ or $\eta_{c}\left(\chi_{b}\right.$ or $\left.\eta_{b}\right)$. This decay could be viewed as fragmentation of gluons $g \rightarrow H_{1}, H_{2}$ with energy fraction $z=1$ with sensitivity to glueball production assuming that the mass of $\chi_{c}$ is high enough for the $g g$ intermediate state to dominate. Here, we explore in particular the symmetry relations for the set of decay rates if all $H_{1}$ and $H_{2}$ are taken from $[q \bar{q}]$ multiplets $M_{1}$ and $M_{2}$ respectively. These relations follow from the quark states being produced as flavour singlet.

A nice example is given by the results in table 4 on $\chi_{c}$ decays where the properly weighted decay rates into pairs of pseudoscalars follow a simple equipartition pattern. The same pattern should be followed by all pairs of mesons $H_{1}, H_{2}$ taken from the same multiplets $M_{1}, M_{2}$ each, i.e. for the branching ratios $B$ into the members of two nonets after summing over charges

$$
B: \quad[Q \bar{Q}] \rightarrow \pi_{1} \pi_{2}: K_{1} \bar{K}_{2}: \bar{K}_{1} K_{2}: \eta_{1} \eta_{2}: \eta_{1}^{\prime} \eta_{2}^{\prime}=3: 2: 2: 1: 1
$$

with obvious notation. In this way, the classification of hadrons into multiplets can be tested. At the same time any additional gluonic component should become visible by a deviation from the symmetry relations. This will be discussed next for the scalar multiplet.

\subsection{Scalar multiplet according to route 2}

In this case, the scalars $a_{0}(980), f_{0}(980), K_{0}^{*}(1430)$ and $f_{0}(1500)$ belong to the same multiplet. For three channels results on $\chi_{c 0}$ decays have been obtained by BES2 $[208,169]$ which we list in table 15 (taking the values from PDG [4]) and we correct for unseen channels. We take into account furthermore only corrections to the rates due to phase space, but no corrections from quark masses or additional dynamical form factors according to our findings on $\chi_{c}$ decays above within the accuracy of around $10 \%$.

The normalized decay rate for $f_{0} f_{0}$ is found slightly higher but compatible with the rescaled $K_{0}^{*} \bar{K}_{0}^{*}$ rate $(1.64 \pm 0.73$ vs. 1.0$)$, as expected for members of the same

multiplet; $K_{0}^{*}(1430)$ is generally accepted as $q \bar{q}$ state. An increased rate for decay into pairs of $f_{0}$ 's could be indicative of an additional glueball component. The experimental uncertainties are still rather large for definitive conclusions. It is interesting to note the similarity of the two decay rates

$$
\begin{aligned}
& B\left(\chi_{c 0} \rightarrow f_{0}(980) f_{0}(980)\right)=(2.68 \pm 1.21) \times 10^{-3} \\
& B\left(\chi_{c 0} \rightarrow \eta^{\prime}(958) \eta^{\prime}(958)\right)=(2.02 \pm 0.22) \times 10^{-3}
\end{aligned}
$$


Table 15. Branching ratios $B$ of $\chi_{c 0}(3415)\left(0^{++}\right)$into pairs of scalars $K_{0}^{*}(1430)^{0}, f_{0} \equiv$ $f_{0}(980), f_{0}^{\prime} \equiv f_{0}(1500)$ and baryons for comparison (data from PDG [4]) (i) in observed channels, (ii) corrected for unseen decays (isospin, also $B\left(f_{0}(980) \rightarrow \pi \pi\right)=0.75 \pm 0.12$ [169] and $B\left(f_{0}(1500) \rightarrow \pi \pi \& K \bar{K}\right.$ from PDG); (iii) $B /(c q)$ with charge weight $c$ and momentum $q$ after rescaling to the value for $K_{0}^{*}(1430)^{0} \bar{K}_{0}^{*}(1430)^{0}$, together with the resp. theoretical expectations for process $g g$ as in table 4 .

\begin{tabular}{lccccc}
\hline & $K_{0}^{*} \bar{K}_{0}^{*}$ & $f_{0} f_{0}$ & $f_{0}^{\prime} f_{0}^{\prime}$ & $p \bar{p}$ & $\Lambda \bar{\Lambda}$ \\
weight $c$ & 2 & 1 & 1 & & \\
\hline & $\left(\pi^{ \pm} K^{\mp}\right)$ & $\left(\pi^{ \pm} \pi^{\mp}\right)$ & $\left(\pi^{ \pm} K^{\mp}\right)$ & & \\
(i) $B \times 10^{3}$ obs & $0.99_{-0.29}^{+0.40}$ & $0.67 \pm 0.21$ & $<0.05$ & & \\
(ii) $B \times 10^{3}$ corr & $2.23_{-0.65}^{+0.90}$ & $2.68 \pm 1.21$ & $<5.0$ & $0.223 \pm 0.013$ & $0.33 \pm 0.04$ \\
(iii) $B /(c q)$ resc & $1.0_{-0.3}^{+0.4}$ & $1.64 \pm 0.73$ & $<5.2$ & & \\
\hline expect $(\mathrm{R}=1.0)$ & 1. & 1. & 1. & & \\
\hline
\end{tabular}

which again is in line with the two states being parity partners as in route 2 with the same $q \bar{q}$ mixing; the similar decay rates in $J / \psi \rightarrow\left(\eta^{\prime}, f_{0}(980)+V\right)$ with $V=(\phi, \omega)$ have been noted already in [61] (see section 4.2). The observation (127) for a gluonic decay indicates that not only the quark structure but also the gluonic components (if any) are similar in $\eta^{\prime}$ and $f_{0}(980)$. A higher accuracy of the $f_{0}$ rates are certainly desirable.

A critical test for the existence of a scalar $q \bar{q}$ multiplet along route 2 would be the observation

$$
B\left(\chi_{c 0} \rightarrow a_{0}(980) a_{0}(980)\right): B\left(\chi_{c 0} \rightarrow K_{0}^{*}(1430) \bar{K}_{0}^{*}(1430)\right)=3: 4
$$

if all charge states are counted, or 1:1 for single charge states. The rates for $f_{0} f_{0}$ and $f_{0}^{\prime} f_{0}^{\prime}$ could possibly deviate from the symmetry expectations because of gluonic components.

The glueball should appear through a signal from $\chi_{c 0} \rightarrow f_{0}(500) f_{0}(500)$ where $f_{0}(500) / \sigma$ is the broad object peaking at $1 \mathrm{GeV}$. The data in $\chi_{c 0} \rightarrow 4 \pi$ by BES [208] show indeed a considerable broad $\pi \pi$ background. Besides $f_{0}(980)$ there is some enhancement between 1200 and $1500 \mathrm{MeV}$ which could get contributions from $f_{2}(1270)$ and $f_{0}(1500)$ interfering destructively with the background so as to yield a drop of the mass spectrum around $1500 \mathrm{MeV}$. Whether this background corresponds to the broad $f_{0}(500)$ could possibly be found out by observing the interferences with the narrow states $f_{0}(980)$ and $f_{0}(1500)$.

In the decay of $\chi_{c 2}$ [208] a strong signal of decay into $\rho \rho$ shows up. In addition, there appear some bands around $m_{\pi \pi} \sim 500 \mathrm{MeV}$; this could be a signal from $\chi_{c 2} \rightarrow f_{0}(500) f_{0}(500)$ where the mass spectrum is squeezed towards low masses because of the threshold factor $p^{5}$ in cms momentum $p$. This could be a signal from the decay into glueballs.

If $f_{0}(500)$ has a high gluon component its pairwise production should be much favoured over the pairwise production of $K_{0}^{*}(800) / \kappa$ in $\chi_{c 0}$ and $\chi_{c 2}$ decays. 


\subsection{Scalar multiplet according to route 1 - tetraquark expectations}

In this case $\sigma, \kappa, f_{0}(980)$ and $a_{0}(980)$ are included in the lowest mass multiplet. If they are built from diquarks the decay would proceed according to the diagram in figure 3a but with production of diquarks instead of quarks (a contribution from figure 3b has been found negligible from the study of table 4). In this process at cms energy of 3.5 $\mathrm{GeV}$ a formfactor for the diquark should play a role. This is actually observed for the production of baryon pairs which are produced from a primary diquark and a primary quark pair. These rates $(p \bar{p}, \Lambda \bar{\Lambda})$ are seen in table 15 to be an order of magnitude smaller than the rates for $[q \bar{q}]$ scalars like $K_{0}^{*} \bar{K}_{0}^{*}$. One would therefore expect that scalars made up from two diquarks would be produced with a smaller rate yet. This appears to contradict the large rate of $f_{0} f_{0}$ vs. $K_{0}^{*} \bar{K}_{0}^{*}$ in table 15 .

Furthermore for route 1 , one should observe the decays $\sigma \sigma$ and $\kappa \kappa$ with the same rate as $f_{0} f_{0}$ and $a_{0} a_{0}$ for particular charge states. In their analyses the BES Collaboration finds indeed a signal from $\kappa \kappa$ of the order of $K_{0}^{*} \bar{K}_{0}^{*}$; they see signals with $\sigma$ but no signal from $\sigma \sigma$ where they take $\sigma$ according to the observation in $J / \psi \rightarrow \omega \pi \pi$ with a peak at low mass. The results on $\sigma$ and $\kappa$ are considered finally as too uncertain to be transformed into decay rates. In $\chi_{c 2}$ decays there seems to be the channel $\sigma \sigma$ as discussed above, but there is no evidence for the channel $f_{0}(980) f_{0}(980)$. It will be interesting to see, whether the branching ratios follow

$$
B\left(\chi_{c} \rightarrow \kappa \kappa\right): B\left(\chi_{c} \rightarrow \sigma \sigma\right)=4: 1
$$

summed over all charge states, which is a result opposite to route 2 above.

We conclude that the further study of $\chi_{c}$ decays with higher statistics (or even of $\chi_{b}$ decays) has a high potential to clarify the structure of the lowest scalar multiplet and the potential presence of gluonic mesons. The same, of course, is also true for multiplets with other quantum numbers, for example heavier pseudoscalars.

\section{Summary and conclusions}

In a summary of our results we try to answer some questions and we provide a minimal mixing scenario for the scalar mesons with glueball which takes into account some relevant findings.

\subsection{Is there any experimental evidence for the existence of glueballs?}

We have studied different scenarios for a scalar meson spectroscopy which includes the lightest glueball of QCD. None of the schemes considered is without problem. It remains unsatisfactory, if the existence of the glueball as a fundamental prediction of QCD, at the end depends on whether such a controversial state like $f_{0}(1370)$ does exist. We have therefore looked after other criteria as well.

There appears to be one observation which can be considered as a direct hint for the existence of glueballs. That is the observation of several LEP experiments that the 
leading system in a gluon jet has an excess of neutral charge, in one measurement by $40 \%$, if compared with a standard Monte Carlo program. There is a new chance at LHC to follow this line of investigation further by obtaining a larger sample of gluon jets with higher energy to study jets with a larger rapidity gap. This should make the effect clearer and could possibly give a hint towards the relevant glueball mass. Future studies are in a "win-win" situation: either they provide better evidence for gluonic mesons, or else they show a deficiency of the "standard Monte Carlo's" with their hadronization models which would be important to understand as well, as these Monte Carlos are the backbone of the phenomenological studies with jets.

\subsection{Are there supernumerous states in the scalar sector?}

This question is related to the selection of scalar nonets.

i) Extra state $f_{0}(1370)$. With the additional states $f_{0}(1500)$ and $f_{0}(1710)$ nearby there is one state too much (discussed as route 1 and 3). Here our analysis has given a clear answer. We have not found convincing evidence for $f_{0}(1370)$ in any of the 2-body decay channels in the study of a large variety of reactions. In particular, elastic $\pi \pi$ scattering phase shift data put an upper limit of $\lesssim 10 \%$ for its branching ratio into $\pi \pi$. There is room for further studies in the $4 \pi$ channel, but so far the results are controversial. Further experimental investigations are desirable.

ii) Extra state $f_{0}(500)$. In this scenario the $K^{* 0}(800) / \kappa$ is not needed. There is some enhanced interaction at low $K \pi$ energy but the $K \pi$ phase shifts saturate below $40^{\circ}$, very unusual for a resonance. A possible clue could come from the study of symmetry relations for decay rates in $\chi_{c}$ decays.

\subsection{Are there hints towards a gluonic component in isoscalar mesons?}

Our analysis of the production and decay pattern has shown that $f_{0}(1500)$ can hardly be described as a pure $q \bar{q}$ state, a particular solution with a $g g$ contribution is suggested depending on the mixing angle. For $f_{0}(980)$ there is no hint for a strong gluonic component from the $\gamma \gamma$ decay width. The state $f_{0}(500)$ couples to strange and nonstrange quarks but is not "flavour blind" so it could be a mixed gluonic-quarkonic state. A promising tool for further clarification of the flavour structure of isoscalars and their mixing angles are the study of $D, D_{s}$ and especially of $B, B_{s}$ decays.

\subsection{Future studies of symmetry relations in $\chi_{c}$ decays}

Besides the study of leading clusters in gluon jets we find the study of decays of heavy quarkonia like $\chi_{c 0}, \chi_{c 2}$ into pairs of scalar particles particularly interesting. This follows from the success of the symmetry relations for pairs of pseudoscalar particles. Such relations could establish which particles form a multiplet. Deviations from symmetry for the isoscalars would be a hint for glueball. The present results are consistent with $f_{0}(980)$ and $K^{* 0}(1430)$ to be in the same multiplet, $f_{0}(980)$ as tetraquark state is disfavoured. 
Although the treatment of particles with a very large width is difficult, there may be some clues on $\sigma$ and $\kappa$ and their multiplets or gluonic component from such studies.

\subsection{A minimal scenario for scalar quarkonium-glueball spectroscopy}

Finally we present a solution which takes into account the main results on the flavour structure of the $f_{0}$ mesons discussed in this report. It is based on the $q \bar{q}$ nonet of route 2 , i.e. it includes $f_{0}(980), a_{0}(980), K^{* 0}(1430), f_{0}(1500)$ with a flavour mixing similar to the mixing of $\eta, \eta^{\prime}$. The near singlet flavour structure of $f_{0}(980)$ has been strongly supported with a mixing angle around $30^{\circ}$ (as compared to $42^{\circ}$ for the pseudoscalars). Also the relative negative sign of the $s \bar{s}$ component within both $f_{0}$ states is established. In that case, $f_{0}(1500)$ needs a contribution from $g g$ to explain the decay branching ratios $(\pi \pi, K \bar{K}, \eta \eta)$ with their small errors. At the same time, $f_{0}(500)$ does not look like a pure glue state, as it doesn't seem to realize flavour symmetry in the decays. The interpretation of $\gamma \gamma$ decays, sometimes with large errors, is not without controversy because of the model dependence. Our findings on the flavour structure suggest as a minimal solution the existence of a glueball which is strongly mixed into $f_{0}(500-1000)$ and $f_{0}(1500)$ whereas $f_{0}(980)$ remains a quarkonium state. We recall that in the physical region the maximal strength of $\pi \pi$ scattering is near $1000 \mathrm{MeV}$ (and not $500 \mathrm{MeV}$ ) with a comparable width. In this sense the broad $f_{0}(500-1000)$ and the narrow $f_{0}(1500)$ overlap in mass, a situation reminiscent of the $\rho-\omega$ system, apart for the very different widths. This suggests a mixing

$$
\begin{array}{ll}
\left|f_{0}(500-1000)\right\rangle & =\sin \phi_{G}|q \bar{q}\rangle-\cos \phi_{G}|g g\rangle \\
\left|f_{0}(1500)\right\rangle & =\cos \phi_{G}|q \bar{q}\rangle+\sin \phi_{G}|g g\rangle \\
|q \bar{q}\rangle & =\cos \phi_{s c}|n \bar{n}\rangle-\sin \phi_{s c}|s \bar{s}\rangle
\end{array}
$$

We prefer for $f_{0}(1500)$ the solution $\tan \phi_{G}>0$ and especially with our value of $\phi_{s c}$

$$
\phi_{s c}=(30 \pm 3)^{\circ}, \quad \phi_{G} \sim 35^{\circ} .
$$

This may be compared with maximal $q \bar{q}-g g$ mixing at $\phi_{G} \sim 45^{\circ}$ while the model [61] corresponds to $\phi_{G}=0$. The result on $\phi_{G}$ assumes a relation between the decay couplings $\left(g_{0} \approx G_{0}\right)$; more generally, one may introduce an extra parameter $r_{G 0}=G_{0} / g_{0}$, for now $r_{G 0} \approx 1$. The flavour structure of $f_{0}(1500)$ with dominant $g g$ and $q \bar{q}$ octet components is also comparable to the models $[35,51]$ (see $(26)$ ) but with a very different multiplet structure.

Specific experimental tests of the presented scenario include the better determination of mixing angles of the $f_{0}$ mesons and their gluonic components. Also the amplitude signs lead to observable effects. A special property of $f_{0}(500-1000)$ in this scheme is the negative coupling of the $s \bar{s}$ component with respect to the one of $f_{0}(980)$ which is indicated in the Argand diagram of figure 13.

A new possibility for glueball spectroscopy is opened up by the gluonic coupling of $f_{0}(500) / \sigma$ and its dominant decay into $\pi \pi$. Because of this coupling a higher mass 
glueball should have a favoured decay into $\sigma \sigma$. In case of $f_{0}(1500)$, which has a probability $1 / 3$ for a gluonic component according to the above simple estimate, there is a decay $\mathrm{BR}$ of around $13 \%$ into $\sigma \sigma$ according to table 12 ; the evidence is particularly clear and unique for the decay channel $\sigma \rightarrow \pi^{0} \pi^{0}$.

As an interesting aspect of the scenario presented for scalar spectroscopy we note the similarity to the recent solution from QCD sum rules for the scalar sector which suggests two gluonic contributions at 1000 and $1500 \mathrm{MeV}$, but with strong correlation. Also there is one group with a similar result from lattice calculations, but alternative views exist as well.

\section{Acknowledgments}

I would like to express my gratitude to Peter Minkowski for the long term collaboration on gluonic mesons, for his inspiration and the theoretical insights he shared with me. I am much obliged to Alan Martin for suggesting and supporting this report and for his helpful comments on the manuscript. Also I would like to thank many colleages for exchanges on the topics of this report at various stages, especially I appreciated the collaboration with Gerard Mennessier and Stephan Narison, and the interest and the stimulating discussions with V. V. Anisovich, D. Bugg, B. Buschbeck, S. U. Chung, F. Close, B. Gary, R. Kaminski, E. Klempt, F. Mandl, W. Männer, B. Meadows, J.R. Pelaez, M. Pennington, A. Sarantsev, J. Wosiek and B.S. Zou. Thanks also to Rainer Schicker for inviting me to the WE-Heraeus Summer school in Heidelberg 2011, which initiated this study.

\section{References}

[1] H. Fritzsch and M. Gell-Mann, "Current algebra: Quarks and what else?," Proc. of the XVI International Conference on High Energy Physics, Chicago, 1072, Vol. 2, p. 135 (J.D. Jackson, A. Roberts, ets.), eConf C 720906V2 (1972) 135

[2] H. Fritzsch and P. Minkowski, "Psi Resonances, Gluons and the Zweig Rule," Nuovo Cim. A 30 (1975) 393

[3] C. Amsler, T. De Grand and B. Krusche, in "Quark Model", Ref. [4]

[4] J. Beringer et al. (Particle Data Group), Phys. Rev. D 86 (2012) 010001

[5] E. Klempt and A. Zaitsev, "Glueballs, Hybrids, Multiquarks. Experimental facts versus QCD inspired concepts," Phys. Rept. 454 (2007) 1

[6] V. Mathieu, N. Kochelev and V. Vento, "The Physics of Glueballs," Int. J. Mod. Phys. E 18 (2009) 1

[7] V. Crede and C. A. Meyer, "The Experimental Status of Glueballs," Prog. Part. Nucl. Phys. 63 (2009) 74

[8] L. D. Landau, Dokl. Akad. Nauk SSSR 60 (1948) 207 On the angular momentum of a two-photon system

[9] C. N. Yang, "Selection Rules for the Dematerialization of a Particle Into Two Photons," Phys. Rev. 77 (1950) 242

[10] P. Minkowski, "Central hadron production in crossing of dedicated hadronic beams," Fizika B14 (2005) 79

[11] K. G. Wilson 1974, "Confinement of Quarks," Phys. Rev. D 10 (1974) 2445 
[12] P. Weisz and P. Majumdar, "Lattice gauge theories", Scholarpedia (2012) 7(4):8615

[13] A. S. Kronfeld, "Twenty-first Century Lattice Gauge Theory: Results from the QCD Lagrangian," arXiv:1203.1204 [hep-lat]

[14] R. Sommer, "A New way to set the energy scale in lattice gauge theories and its applications to the static force and alpha-s in SU(2) Yang-Mills theory," Nucl. Phys. B 411 (1994) 839

[15] S. Durr, Z. Fodor, J. Frison, C. Hoelbling, R. Hoffmann, S. D. Katz, S. Krieg and T. Kurth et al., "Ab-Initio Determination of Light Hadron Masses," Science 322 (2008) 1224

[16] J. J. Dudek, R. G. Edwards, B. Joo, M. J. Peardon, D. G. Richards and C. E. Thomas, "Isoscalar meson spectroscopy from lattice QCD," Phys. Rev. D 83 (2011) 111502

[17] G. S. Bali et al. [UKQCD Collaboration], "A Comprehensive lattice study of SU(3) glueballs," Phys. Lett. B 309 (1993) 378

[18] C. J. Morningstar and M. J. Peardon, "The Glueball spectrum from an anisotropic lattice study," Phys. Rev. D 60 (1999) 034509

[19] Y. Chen, A. Alexandru, S. J. Dong, T. Draper, I. Horvath, F. X. Lee, K. F. Liu and N. Mathur et al., "Glueball spectrum and matrix elements on anisotropic lattices," Phys. Rev. D 73 (2006) 014516

[20] A. Hart and M. Teper [UKQCD Collaboration], "Glueball spectrum in $\mathrm{O}(\mathrm{a})$ improved lattice QCD," Phys. Rev. D 65 (2002) 034502

[21] A. Hart et al. [UKQCD Collaboration], "A Lattice study of the masses of singlet 0++ mesons," Phys. Rev. D 74 (2006) 114504

[22] C. M. Richards et al. [UKQCD Collaboration], "Glueball mass measurements from improved staggered fermion simulations," Phys. Rev. D 82 (2010) 034501

[23] E. Gregory, A. Irving, B. Lucini, C. McNeile, A. Rago, C. Richards and E. Rinaldi, "Towards the glueball spectrum from unquenched lattice QCD," JHEP 1210 (2012) 170

[24] M. A. Shifman, A. I. Vainshtein and V. I. Zakharov, "QCD and Resonance Physics. Sum Rules," Nucl. Phys. B 147 (1979) 385

[25] M. A. Shifman, "Snapshots of hadrons or the story of how the vacuum medium determines the properties of the classical mesons which are produced, live and die in the QCD vacuum," Prog. Theor. Phys. Suppl. 131 (1998) 1

[26] P. Colangelo and A. Khodjamirian, "QCD sum rules, a modern perspective," In *Shifman, M. (ed.): At the frontier of particle physics, vol. $3^{*} 1495-1576$

[27] V. A. Novikov, M. A. Shifman, A. I. Vainshtein and V. I. Zakharov, "Are All Hadrons Alike?," Nucl. Phys. B 191 (1981) 301

[28] T. Schafer and E. V. Shuryak, "Glueballs and instantons," Phys. Rev. Lett. 75 (1995) 1707

[29] S. Narison, "Spectral Function Sum Rules For Gluonic Currents," Z. Phys. C 26 (1984) 209

[30] S. Narison and G. Veneziano, "QCD Tests Of G (1.6) = Glueball," Int. J. Mod. Phys. A 4 (1989) 2751

[31] S. Narison, "Light scalar mesons in QCD," Nucl. Phys. Proc. Suppl. 186 (2009) 306

[32] S. Narison, "QCD tests of the puzzling scalar mesons," Phys. Rev. D 73 (2006) 114024

[33] H. Forkel, "Direct instantons, topological charge screening and QCD glueball sum rules," Phys. Rev. D 71 (2005) 054008

[34] D. Harnett, R. T. Kleiv, K. Moats and T. G. Steele, "Near-Maximal Mixing of Scalar Gluonium and Quark Mesons: A Gaussian Sum-Rule Analysis," Nucl. Phys. A 850 (2011) 110

[35] C. Amsler and F. E. Close, "Is f0 (1500) a scalar glueball?," Phys. Rev. D 53 (1996) 295; "Evidence for a a scalar glueball," Phys. Lett. B 353 (1995) 385

[36] V. V. Anisovich, Y. .D. Prokoshkin and A. V. Sarantsev, "Nonet classification of scalar / isoscalar resonances below 1900-MeV: The Existence of an extra scalar state in the region 1200-MeV 1600-MeV," Phys. Lett. B 389 (1996) 388

[37] V. V. Anisovich and A. V. Sarantsev, "K matrix analysis of the (I $\left.\mathrm{J}^{* *}(\mathrm{PC})=00++\right)$-wave in the mass region below $1900 \mathrm{MeV}$," Eur. Phys. J. A 16 (2003) 229

[38] Q. Zhao, "A Coherent study of chi $(\mathrm{c} 0,2) \rightarrow$ VV, PP and SS," Phys. Rev. D 72 (2005) 074001 
[39] M. Chanowitz, "Chiral suppression of scalar glueball decay," Phys. Rev. Lett. 95 (2005) 172001

[40] C. Di Donato, G. Ricciardi and I. Bigi, " $\eta-\eta^{\prime}$ Mixing - From electromagnetic transitions to weak decays of charm and beauty hadrons," Phys. Rev. D 85 (2012) 013016

[41] T. Feldmann, P. Kroll and B. Stech, "Mixing and decay constants of pseudoscalar mesons," Phys. Rev. D 58 (1998) 114006

[42] T. Feldmann, P. Kroll and B. Stech, "Mixing and decay constants of pseudoscalar mesons: The Sequel," Phys. Lett. B 449 (1999) 339

[43] G. S. Adams et al. [CLEO Collaboration], "chi(c0) and chi(c2) Decays into eta eta, eta eta-prime, and eta-prime eta-prime Final States," Phys. Rev. D 75 (2007) 071101

[44] J. Z. Bai et al. [BES Collaboration], "Radiative decay of the psi(2S) into two pseudoscalar mesons," Phys. Rev. D 67 (2003) 032004

[45] D. Robson, "A Basic Guide for the Glueball Spotter," Nucl. Phys. B 130 (1977) 328

[46] F. E. Close, "Gluonic Hadrons," Rept. Prog. Phys. 51 (1988) 833

[47] V. A. Khoze, A. D. Martin and M. G. Ryskin, "Prospects for new physics observations in diffractive processes at the LHC and Tevatron," Eur. Phys. J. C 23 (2002) 311

[48] P. Minkowski and W. Ochs, "B decays into light scalar particles and glueball," Eur. Phys. J. C 39 (2005) 71

[49] V. V. Anisovich et al. [Crystal Ball. Collaboration], "Observation of two $\mathrm{J}(\mathrm{PC})=0++$ isoscalar resonances at 1365-MeV and 1520-MeV," Phys. Lett. B 323 (1994) 233

[50] D. Alde et al. [Serpukhov-Brussels-Los Alamos-Annecy(LAPP)-Pisa Collaboration], "PRODUCTION OF G (1590) in 300-GeV CENTRAL pi- N COLLISIONS," Phys. Lett. B 201 (1988) 160 [Yad. Fiz. 47 (1988) 1639] [Sov. J. Nucl. Phys. 47 (1988) 1038]

[51] F. E. Close, G. R. Farrar and Z. -p. Li, "Determining the gluonic content of isoscalar mesons," Phys. Rev. D 55 (1997) 5749

[52] F. Giacosa, T. Gutsche, V. E. Lyubovitskij and A. Faessler, "Scalar nonet quarkonia and the scalar glueball: Mixing and decays in an effective chiral approach," Phys. Rev. D 72 (2005) 094006

[53] H. -Y. Cheng, C. -K. Chua and K. -F. Liu, "Scalar glueball, scalar quarkonia, and their mixing," Phys. Rev. D 74 (2006) 094005

[54] D. Morgan, "Is the 0+ Nonet Respectable?," Phys. Lett. B 51 (1974) 71

[55] N. A. Tornqvist and M. Roos, "Resurrection of the sigma meson," Phys. Rev. Lett. 76 (1996) 1575

[56] E. van Beveren and G. Rupp, "Comment on 'Understanding the scalar meson q anti-q nonet'," Eur. Phys. J. C 10 (1999) 469

[57] R. L. Jaffe, "Multi-Quark Hadrons. 1. The Phenomenology of (2 Quark 2 anti-Quark) Mesons," Phys. Rev. D 15 (1977) 267; D 15 (1977) 281

[58] N. N. Achasov and V. N. Ivanchenko, "On a Search for Four Quark States in Radiative Decays of phi Meson," Nucl. Phys. B 315 (1989) 465

[59] N. N. Achasov and G. N. Shestakov, "Light Scalar Mesons in Photon-Photon Collisions," Phys. Usp. 54 (2011) 799;

N. N. Achasov and G. N. Shestakov, "Study of light scalars in Photon-Photon Collisions, the learned lessons" Nucl. Phys. B (Proc. Suppl.) 225-227 (2012) 135

[60] G. 't Hooft, G. Isidori, L. Maiani, A. D. Polosa and V. Riquer, "A Theory of Scalar Mesons," Phys. Lett. B 662 (2008) 424

[61] P. Minkowski and W. Ochs, "Identification of the glueballs and the scalar meson nonet of lowest mass," Eur. Phys. J. C 9 (1999) 283

[62] P. Minkowski, "Sigma Models With Sigmas And The Long Range Aspect Of The Delta I = 1/2 Rule," Nucl. Phys. Proc. Suppl. 7A (1989) 118

[63] K. L. Au, D. Morgan and M. R. Pennington, "Meson Dynamics Beyond the Quark Model: A Study of Final State Interactions," Phys. Rev. D 35 (1987) 1633

[64] E. Klempt, B. C. Metsch, C. R. Munz and H. R. Petry, "Scalar mesons in a relativistic quark 
model with instanton induced forces," Phys. Lett. B 361 (1995) 160

[65] M. Koll, R. Ricken, D. Merten, B. C. Metsch and H. R. Petry, "A Relativistic quark model for mesons with an instanton induced interaction," Eur. Phys. J. A 9 (2000) 73;

R. Ricken, M. Koll, D. Merten, B. C. Metsch and H. R. Petry, "The Meson spectrum in a covariant quark model," Eur. Phys. J. A 9 (2000) 221

[66] V. Dmitrasinovic, "U-A(1) breaking and scalar mesons in the Nambu-Jona-Lasinio model," Phys. Rev. C 53 (1996) 1383

[67] G. 't Hooft, "Computation of the Quantum Effects Due to a Four-Dimensional Pseudoparticle," Phys. Rev. D 14 (1976) 3432 [Erratum-ibid. D 18 (1978) 2199]

[68] M. Ablikim et al., "Partial wave analyses of J/psi $\rightarrow$ gamma pi+ pi- and gamma pi0 pi0," Phys. Lett. B 642 (2006) 441

[69] Guangming Xu [BES III Collaboration], $X^{\text {th }}$ Quark Confinement and the Hadron Spectrum, Munich, Germany, October 2012, Proc. of Science, to be published.

[70] P. Minkowski and W. Ochs, "On the scalar nonet lowest in mass," Nucl. Phys. Proc. Suppl. 121 (2003) 119

[71] A. V. Anisovich, V. A. Nikonov, A. V. Sarantsev, V. V. Anisovich, M. A. Matveev, T. O. Vulfs, K. V. Nikonov and J. Nyiri, "Analysis of the meson-meson data in the framework of the dispersion D-matrix method," Phys. Rev. D 84 (2011) 076001

[72] A. Sirlin, "Theoretical considerations concerning the Z0 mass," Phys. Rev. Lett. 67 (1991) 2127; B. A. Kniehl and A. Sirlin, "Pole Mass, Width, and Propagators of Unstable Fermions," Phys. Rev. D 77 (2008) 116012

[73] P. Minkowski, "On concise hypotheses for the interpretation of a wide scalar resonance as gauge boson binary in QCD: Some new analyses," Nucl. Phys. Proc. Suppl. 186 (2009) 302

P. Minkowski, Conference in Honor of Murray Gell-Mann's 80th Birthday: Quantum Mechanics, Elementary Particles, Quantum Coismology \& Complexity, 24-26 Feb 2010, Singapore, HEP records C10-02-24, p.74-88 "QCD - mass and gauge in a field theory: QCD glue mesons,"

[74] S. M. Roy, "Exact integral equation for pion pion scattering involving only physical region partial waves," Phys. Lett. B 36 (1971) 353

[75] I. Caprini, G. Colangelo and H. Leutwyler, "Mass and width of the lowest resonance in QCD," Phys. Rev. Lett. 96 (2006) 132001

[76] R. Garcia-Martin, R. Kaminski, J. R. Pelaez and J. Ruiz de Elvira, "Precise determination of the $\mathrm{f0}(600)$ and $\mathrm{f0}(980)$ pole parameters from a dispersive data analysis," Phys. Rev. Lett. 107 (2011) 072001

[77] B. Moussallam, "Couplings of light $\mathrm{I}=0$ scalar mesons to simple operators in the complex plane," Eur. Phys. J. C 71 (2011) 1814

[78] C. Amsler, S. Eidelman, T. Gutsche, C. Hanhart, S.Spanier, N.A. Törnqvist, "Note on scalar mesons below 2 GeV", 2012, Ref. [4]

[79] G. Mennessier, "Meson Pair Production in gamma Gamma Scattering and Implications for Scalar Mesons," Z. Phys. C 16 (1983) 241

[80] G. Mennessier, S. Narison and W. Ochs, "Glueball nature of the sigma / f(0)(600) from pi pi and gamma gamma scatterings," Phys. Lett. B 665 (2008) 205;

G. Mennessier, P. Minkowski, S. Narison and W. Ochs, "Can the gamma-gamma processes reveal the nature of the sigma meson?," SLAC Econf C0709107

[81] G. Mennessier, S. Narison and X. G. Wang, "The sigma and $f_{0}(980)$ from Ke4 + pi-pi scatterings data," Phys. Lett. B 688 (2010) 59;

G. Mennessier, S. Narison and X. -G. Wang, "sigma and $f_{0}(980)$ substructures from gammagamma to pi-pi, J/psi, phi radiative and $D_{s}$ semi-leptonic decays," Phys. Lett. B 696 (2011) 40

[82] A. Garmash et al. [Belle Collaboration], "Dalitz analysis of the three-body charmless decays B+ $\rightarrow \mathrm{K}+$ pi+ pi- and B+ $\rightarrow \mathrm{K}+\mathrm{K}+\mathrm{K}-$, , Phys. Rev. D 71 (2005) 092003 
[83] R. Aaij et al. [LHCb Collaboration], "Analysis of the resonant components in $B_{s} \rightarrow J / \psi \pi^{+} \pi^{-}$," Phys. Rev. D 86 (2012) 052006;

"First observation of $B_{s} \rightarrow J / \psi f_{0}(980)$ decays," Phys. Lett. B 698 (2011) 115

[84] J. E. Augustin et al. [DM2 Collaboration], "Study Of The J / Psi Decay Into Five Pions," Nucl. Phys. B 320 (1989) 1

[85] M. Ablikim et al. [BES Collaboration], "The sigma pole in J / psi $\rightarrow$ omega pi+ pi-," Phys. Lett. B 598 (2004) 149

[86] E. M. Aitala et al. [E791 Collaboration], "Experimental evidence for a light and broad scalar resonance in D+ $\rightarrow$ pi- pi+ pi+ decay," Phys. Rev. Lett. 86 (2001) 770

[87] F. Q. Wu and B. S. Zou, "Subthreshold rho contribution in J/psi decay to omega pi pi and K anti-K pi," Phys. Rev. D 73 (2006) 114008

[88] W. Ochs, "Light scalar meson spectrum," AIP Conf. Proc. 619 (2002) 167 [hep-ph/0111309].

[89] R. Kaminski, G. Mennessier and S. Narison, "Gluonium nature of the sigma/f(0)(600) from its coupling to K anti-K," Phys. Lett. B 680 (2009) 148

[90] D. V. Bugg, "Reconciling phi radiative decays with other data for a0(980), fo(980), pi pi $\rightarrow$ KK and pi pi $\rightarrow$ eta eta," Eur. Phys. J. C 47 (2006) 45

[91] A. Etkin et al., "Amplitude Analysis of the K0(s) K0(s) System Produced in the Reaction pi- p $\rightarrow \mathrm{K} 0(\mathrm{~s}) \mathrm{K} 0(\mathrm{~s}) \mathrm{n}$ at $23-\mathrm{GeV} / \mathrm{c}$," Phys. Rev. D 25 (1982) 1786

[92] D. H. Cohen, D. S. Ayres, R. Diebold, S. L. Kramer, A. J. Pawlicki and A. B. Wicklund, "Amplitude Analysis of the K- K+ System Produced in the Reactions pi- p $\rightarrow \mathrm{K}-\mathrm{K}+\mathrm{n}$ and pi+ n $\rightarrow$ K- K+ p at 6-GeV/c," Phys. Rev. D 22 (1980) 2595

[93] N. N. Achasov and A. V. Kiselev, "Analytical $\pi \pi$ scattering amplitude and the light scalars-II," Phys. Rev. D 85 (2012) 094016

[94] S. Uehara et al. [Belle Collaboration], "High-statistics measurement of neutral pion-pair production in two-photon collisions," Phys. Rev. D 78 (2008) 052004

[95] T. Mori et al. [Belle Collaboration], "High statistics study of $\mathrm{f}(0)(980)$ resonance in gamma gamma $\rightarrow$ pi+ pi- production," Phys. Rev. D 75 (2007) 051101

[96] M. R. Pennington, "Sigma coupling to photons: Hidden scalar in gamma gamma $\rightarrow$ pi0 pi0," Phys. Rev. Lett. 97 (2006) 011601

[97] S. Narison, "Masses, decays and mixings of gluonia in QCD," Nucl. Phys. B 509 (1998) 312

[98] R. Omnes, "On the Solution of certain singular integral equations of quantum field theory," Nuovo Cim. 8 (1958) 316;

N. I. Muskhelishvili, Singular Integral Equations, (Amsterdam, North Holland, 1958)

[99] C. Michael, Phys. Lett. 21 (1966) 93

[100] R. Garcia-Martin, R. Kaminski, J. R. Pelaez, J. Ruiz de Elvira and F. J. Yndurain, "The Pionpion scattering amplitude. IV: Improved analysis with once subtracted Roy-like equations up to $1100 \mathrm{MeV}$," Phys. Rev. D 83 (2011) 074004

[101] B. Hyams et al. [CERN-Munich Collaboration], "pi pi Phase Shift Analysis from 600-MeV to 1900-MeV," Nucl. Phys. B 64 (1973) 134;

W. Ochs, thesis, Ludwig-Maximilians-University, Munich, 1973

[102] W. Ochs, "Scalar mesons: In search of the lightest glueball," Nucl. Phys. Proc. Suppl. 174 (2007) 146

[103] P. del Amo Sanchez et al. [The BABAR Collaboration], "Analysis of the $D^{+} \rightarrow K^{-} \pi^{+} e^{+} \nu_{e}$ decay channel," Phys. Rev. D 83 (2011) 072001

[104] W. Dunwoodie, quoted in [103]

[105] D. Aston et al. [LASS Collaboration] "A Study of K- pi+ Scattering in the Reaction K- p $\rightarrow$ Kpi+ n at 11-GeV/c," Nucl. Phys. B 296 (1988) 493

[106] P. Estabrooks, R. K. Carnegie, A. D. Martin, W. M. Dunwoodie, T. A. Lasinski and D. W. G. S. Leith, "Study of K pi Scattering Using the Reactions K+- p $\rightarrow \mathrm{K}+-\mathrm{pi}+\mathrm{n}$ and K+- p $\rightarrow$ K+- pi- Delta++ at 13-GeV/c," Nucl. Phys. B 133 (1978) 490

[107] K. M. Watson, "Some general relations between the photoproduction and scattering of pi mesons," 
Phys. Rev. 95 (1954) 228

[108] J. M. Link et al. [FOCUS Collaboration], "Hadronic mass spectrum analysis of D+ $\rightarrow \mathrm{K}-\mathrm{pi}+$ mu+ nu decay and measurement of the $\mathrm{K}^{*}(892) 0$ mass and width," Phys. Lett. B 621 (2005) 72

[109] D. Epifanov et al. [Belle Collaboration], "Study of tau- $\rightarrow \mathrm{K}(\mathrm{S})$ pi- nu(tau) decay at Belle," Phys. Lett. B 654 (2007) 65

[110] S. Paramesvaran [BaBar Collaboration], "Selected topics in tau physics from BaBar," arXiv:0910.2884 [hep-ex].

[111] S. Descotes-Genon and B. Moussallam, "The $\mathrm{K}^{*} 0$ (800) scalar resonance from Roy-Steiner representations of pi K scattering," Eur. Phys. J. C 48 (2006) 553

[112] S. Ishida, M. Ishida, T. Ishida, K. Takamatsu and T. Tsuru, "Analysis of K pi scattering phase shift and existence of kappa (900) particle," Prog. Theor. Phys. 98 (1997) 621

[113] V. V. Anisovich, V. A. Nikonov and L. Montanet, "Quark content of f0(980) from the decay ratios into pi pi, K anti-K and gamma gamma," Phys. Lett. B 480 (2000) 19

[114] R. Fleischer, R. Knegjens and G. Ricciardi, "Anatomy of $B_{s, d}^{0} \rightarrow J / \psi f_{0}(980)$," Eur. Phys. J. C 71 (2011) 1832

[115] S. M. Flatte, "Coupled - Channel Analysis of the pi eta and K anti-K Systems Near K anti-K Threshold," Phys. Lett. B 63 (1976) 224

[116] M. Ablikim et al. [BES Collaboration], "Resonances in J / psi $\rightarrow$ phi pi+ pi- and phi K+ K-," Phys. Lett. B 607 (2005) 243

[117] A. D. Martin, E. N. Ozmutlu and E. J. Squires, "The pi pi and K anti-K Amplitudes, the S* and the Quark Structure of 0++ Resonances," Nucl. Phys. B 121 (1977) 514

[118] P. Estabrooks, "Where and What Are the Scalar Mesons?," Phys. Rev. D 19 (1979) 2678

[119] D. V. Bugg, B. S. Zou and A. V. Sarantsev, "New results on pi pi phase shifts between 600-MeV and 1900-MeV," Nucl. Phys. B 471 (1996) 59

[120] R. Kaminski, L. Lesniak and B. Loiseau, "Scalar mesons and multichannel amplitudes," Eur. Phys. J. C 9 (1999) 141

[121] S. Uehara et al. [Belle Collaboration], "High-statistics study of eta pi0 production in two-photon collisions," Phys. Rev. D 80 (2009) 032001

[122] B. Aubert et al. [BABAR Collaboration], "Dalitz Plot Analysis of $D_{s}+\rightarrow \pi^{+} \pi^{-} \pi^{+}$," Phys. Rev. D 79 (2009) 032003

[123] P. del Amo Sanchez et al. [BABAR Collaboration], "Dalitz plot analysis of $D_{s}^{+} \rightarrow K^{+} K^{-} \pi^{+}$," Phys. Rev. D 83 (2011) 052001

[124] R. E. Mitchell et al. [CLEO Collaboration], "Dalitz Plot Analysis of D+(s) $\rightarrow \mathrm{K}+\mathrm{K}-\mathrm{pi}+$," Phys. Rev. D 79 (2009) 072008

[125] D. V. Bugg, "A Study in Depth of f0(1370)," Eur. Phys. J. C 52 (2007) 55

[126] C. Amsler et al. [Crystal Barrel Collaboration], "Coupled channel analysis of anti-p p annihilation into pi0 pi0 pi0, pi0 Phys. Lett. B 355 (1995) 425

[127] C. Amsler et al. [Crystal Barrel Collaboration], "High statistics study of f0 (1500) decay into pi0 pi0," Phys. Lett. B 342 (1995) 433

[128] C. Amsler et al. [Crystal Barrel Collaboration], "Proton anti-proton annihilation at 900-MeV/c into pi0 pi0 pi0, pi0 pi0 eta and pi0 eta eta," Eur. Phys. J. C 23 (2002) 29

[129] T. A. Armstrong et al. [E760 Collaboration], "Evidence for $\eta \eta$ resonances in $\bar{p} p$ annihilations at 2950 i $\sqrt{s}$; 3620-MeV," Phys. Lett. B 307 (1993) 394

[130] I. Uman, D. Joffe, Z. Metreveli, K. K. Seth, A. Tomaradze and P. K. Zweber, "Light quark resonances in p anti-p annihilations at 5.2-GeV/c," Phys. Rev. D 73 (2006) 052009

[131] G. F. Chew and F. E. Low, "Unstable particles as targets in scattering experiments," Phys. Rev. 113 (1959) 1640

[132] B.R. Martin, D. Morgan and G. Shaw, "Pion Pion Interactions in Particle Physics", Academic Press, London, 1976

[133] W. Ochs, "A method for the determination of density matrix elements and amplitudes of one-pion 
exchange reactions," Nuovo Cim. A 12 (1972) 724

[134] P. Estabrooks and A. D. Martin, "Analysis of pi- $\mathrm{p} \rightarrow$ pi- pi+ n data at $17.2 \mathrm{GeV} / \mathrm{c}$," Phys. Lett. B 41 (1972) 350

[135] W. Ochs and F. Wagner, "On the strength of absorption in single pion production reactions," Phys. Lett. B 44 (1973) 271

[136] P. Estabrooks and A. D. Martin, "pi pi Phase Shift Analysis Below the K anti-K Threshold," Nucl. Phys. B 79 (1974) 301

[137] G. Grayer et al. [CERN-Munich Collaboration], "High Statistics Study of the Reaction pi- p $\rightarrow$ pi- pi+ n: Apparatus, Method of Analysis, and General Features of Results at 17-GeV/c," Nucl. Phys. B 75 (1974) 189

[138] G. Grayer et al. [CERN-Munich Collaboration], "Rho-polarization in the reaction pi- p $\rightarrow$ pi+ pi- n at 17 gev," Nucl. Phys. B 50 (1972) 29

[139] S. U. Chung, "Techniques of amplitude analysis for two pseudoscalar systems," Phys. Rev. D 56 (1997) 7299

[140] E. Barrelet, "A new point of view in the analysis of two-body reactions," Nuovo Cim. A 8 (1972) 331

[141] H. Becker et al. [CERN-Munich Collaboration], "Measurement And Analysis Of The Reaction Pi- P $\rightarrow$ Rho0 N On A Polarized Target," Nucl. Phys. B 150 (1979) 301

[142] H. Becker et al. [CERN-Cracow-Munich Collaboration], "A Model Independent Partial Wave Analysis of the pi+ pi- System Produced at Low Four Momentum Transfer in the Reaction pip (Polarized) $\rightarrow$ pi+ pi- n at 17.2-GeV/c," Nucl. Phys. B 151 (1979) 46

[143] P. Estabrooks and A. D. Martin, "pi pi Phase Shift Analysis in the f and g Resonance Regions," Phys. Lett. B 53 (1974) 253

[144] P. Estabrooks and A. D. Martin, "pi pi Partial Waves from 0.6-GeV to 1.8-GeV," Nucl. Phys. B 95 (1975) 322

[145] A. D. Martin and M. R. Pennington, "How Imposing Analyticity on a pi pi Phase Shift Analysis Can Reveal New Solutions, Explore Experimental Structures and Investigate the Possibility of New Resonances," Annals Phys. 114 (1978) 1.

[146] B. Hyams et al. [CERN-Munich Collaboration], "A Study of All the pi pi Phase Shift Solutions in the Mass Region 1.0-GeV to 1.8-GeV from pi- $\mathrm{p} \rightarrow$ pi- pi+ $\mathrm{n}$ at $17.2-\mathrm{GeV}$," Nucl. Phys. B $100(1975) 205$

[147] R. Kaminski, J. R. Pelaez and F. J. Yndurain, "The pion-pion scattering amplitude. II. Improved analysis above bar K anti-K threshold," Phys. Rev. D 74 (2006) 014001 [Erratum-ibid. D 74 (2006) 079903]

[148] R. Kaminski, L. Lesniak and K. Rybicki, "Separation of S wave pseudoscalar and pseudovector amplitudes in pi- p (polarized) $\rightarrow$ pi+ pi- $\mathrm{n}$ reaction on polarized target," Z. Phys. C 74 (1997) 79

[149] J. Gunter et al. [E852 Collaboration], "A Partial wave analysis of the pi0 pi0 system produced in pi- p charge exchange collisions," Phys. Rev. D 64 (2001) 072003

[150] D. Alde et al. [GAMS Collaboration], "Study of the pi0 pi0 system with the GAMS-4000 spectrometer at 100-GeV/c," Eur. Phys. J. A 3 (1998) 361

[151] W. Ochs, "No Indication of f0(1370) in pi pi Phase Shift Analyses," AIP Conf. Proc. 1257 (2010) 252

[152] N. B. Durusoy, M. Baubillier, R. George, M. Goldberg, A. M. Touchard, N. Armenise, M. T. Fogli Muciaccia and A. Silvestri, "Study of the $\mathrm{i}=2$ pi pi scattering from the reaction pi- $\mathrm{d} \rightarrow$ pi- pip(s) p at 9.0 gev/c," Phys. Lett. B 45 (1973) 517

[153] D. H. Cohen, T. Ferbel, P. Slattery and B. Werner, "Study Of Pi Pi Scattering In The Isotopic Spin-2 Channel," Phys. Rev. D 7 (1973) 661.

[154] P. Buettiker, S. Descotes-Genon and B. Moussallam, "A new analysis of pi K scattering from Roy and Steiner type equations," Eur. Phys. J. C 33 (2004) 409

[155] N. O. Johannesson and J. L. Petersen, "Coupled channel study of the S wave pi pi K anti-K 
interaction," Nucl. Phys. B 68 (1974) 397.

[156] F. G. Binon et al. [Serpukhov-Brussels-Annecy(LAPP) Collaboration], "G (1590): A Scalar Meson Decaying Into Two eta Mesons," Nuovo Cim. A 78 (1983) 313

[157] F. G. Binon et al. [GAMS Collaboration], "Investigation of the eta eta system in pi- p interactions at a momentum of 32.5-GeV/c at the GAMS-4pi spectrometer," Phys. Atom. Nucl. 68 (2005) 960 [Yad. Fiz. 68 (2005) 998]

[158] E. M. Aitala et al. [E791 Collaboration], "Model independent measurement of S-wave K- pi+ systems using D+ $\rightarrow$ K pi pi decays from Fermilab E791," Phys. Rev. D 73 (2006) 032004 [Erratum-ibid. D 74 (2006) 059901]

[159] J. M. Link et al. [FOCUS Collaboration], "Dalitz plot analysis of $\mathrm{D}(\mathrm{s})+$ and $\mathrm{D}+$ decay to pi+ pi- pi+ using the K matrix formalism," Phys. Lett. B 585 (2004) 200

[160] E. Klempt, M. Matveev and A. V. Sarantsev, "Further study of D+(s) decays into pi- pi+ pi+," Eur. Phys. J. C 55 (2008) 39

[161] E. M. Aitala et al. [E791 Collaboration], "Study of the $\mathrm{D}+(\mathrm{s}) \rightarrow$ pi- pi+ pi+ decay and measurement of $\mathrm{f}(0)$ masses and widths," Phys. Rev. Lett. 86 (2001) 765

[162] B. Aubert et al. [BABAR Collaboration], "Dalitz plot analysis of the decay $B^{ \pm} \rightarrow K^{ \pm} K^{ \pm} K^{\mp}$," Phys. Rev. D 74 (2006) 032003

[163] J. Li et al. [Belle Collaboration], "Observation of $B_{s}^{0} \rightarrow J / \psi f_{0}(980)$ and Evidence for $B_{s}^{0} \rightarrow$ $J / \psi f_{0}(1370), "$ Phys. Rev. Lett. 106 (2011) 121802

[164] A. Datta, H. J. Lipkin and P. J. O'Donnell, "Simple relations for two-body B decays to charmonium and tests for eta eta-prime mixing," Phys. Lett. B 529 (2002) 93

[165] X. Liu, H. -n. Li and Z. -J. Xiao, "Implications on $\eta$ - $\eta^{\prime}$-glueball mixing from $B_{d / s} \rightarrow J / \Psi \eta^{(\prime)}$ Decays," Phys. Rev. D 86 (2012) 011501

[166] R. Knegjens, "An exploration of $B_{s} \rightarrow J / \psi s \bar{s}$," arXiv:1209.3206 [hep-ph]

[167] R. Aaij et al. [LHCb Collaboration], "Observation of $B_{s} \rightarrow J / \psi f_{2}^{\prime}(1525)$ in $J / \psi K^{+} K^{-}$final states," Phys. Rev. Lett. 108 (2012) 151801

[168] J. Li et al. [Belle Collaboration], "First observation of $B_{s}^{0} \rightarrow J / \psi \eta$ and $B_{s}^{0} \rightarrow J / \psi \eta^{\prime}$," Phys. Rev. Lett. 108 (2012) 181808

[169] M. Ablikim et al. [BES Collaboration], "Partial wave analysis of chi $(\mathrm{c} 0) \rightarrow \mathrm{pi}+\mathrm{pi}-\mathrm{K}+\mathrm{K}-$," Phys. Rev. D 72 (2005) 092002

[170] D. Barberis et al. [WA102 Collaboration], "A Study of the eta eta channel produced in central p p interactions at 450-GeV/c," Phys. Lett. B 479 (2000) 59

[171] T. Akesson et al. [Axial Field Spectrometer Collaboration], "A Search For Glueballs And A Study Of Double Pomeron Exchange At The Cern Intersecting Storage Rings," Nucl. Phys. B 264 (1986) 154

[172] D. Barberis et al. [WA102 Collaboration], "A Partial wave analysis of the centrally produced pi0 pi0 system in p p interactions at 450-GeV/c," Phys. Lett. B 453 (1999) 325

[173] D. Alde et al. [GAMS Collaboration], "Study of the pi0 pi0 system in p p central collisions at 450-GeV/c," Phys. Lett. B 397 (1997) 350

[174] R. Schicker [ALICE Collaboration], "Central Meson Production in ALICE," 14th International Conference on Hadron Spectroscopy: Hadron 2011 13-17 Jun 2011, Munich, Germany, HEP records: C11-06-13;

K. Aamodt et al. [ALICE Collaboration], "The ALICE experiment at the CERN LHC," JINST 3 (2008) S08002

[175] P. Minkowski and W. Ochs, "The Glueball among the light scalar mesons," Nucl. Phys. Proc. Suppl. 121 (2003) 123

[176] P. Lebiedowicz and A. Szczurek, "Exclusive $p p \rightarrow p p \pi^{+} \pi^{-}$reaction: From the threshold to LHC," Phys. Rev. D 81 (2010) 036003

[177] F. E. Close and A. Kirk, "A Glueball - q anti-q filter in central hadron production," Phys. Lett. B 397 (1997) 333

[178] D. Barberis et al. [WA102 Collaboration], "A Coupled channel analysis of the centrally produced 
$\mathrm{K}+\mathrm{K}-$ and pi+ pi- final states in p p interactions at 450-GeV/c," Phys. Lett. B 462 (1999) 462

[179] F. E. Close, A. Kirk and G. Schuler, "Dynamics of glueball and q anti-q production in the central region of p p collisions," Phys. Lett. B 477 (2000) 13

[180] A. B. Kaidalov, V. A. Khoze, A. D. Martin and M. G. Ryskin, "Central exclusive diffractive production as a spin-parity analyser: From Hadrons to Higgs," Eur. Phys. J. C 31 (2003) 387

[181] D. Barberis et al. [WA102 Collaboration], "A Study of the $\mathrm{f}(0)(1370), \mathrm{f}(0)(1500), \mathrm{f}(0)(2000)$ and $\mathrm{f}(2)(1950)$ observed in the centrally produced 4 pi final states," Phys. Lett. B 474 (2000) 423

[182] D. Barberis et al. [WA102 Collaboration], "A Spin analysis of the 4 pi channels produced in central p p interactions at 450-GeV/c," Phys. Lett. B 471 (2000) 440

[183] A. Abele et al. [Crystal Barrel Collaboration], "Study of f0 decays into four neutral pions," Eur. Phys. J. C 19 (2001) 667

[184] C. Amsler, "Further evidence for a large glue component in the $f(0)(1500)$ meson," Phys. Lett. B 541 (2002) 22

[185] V. V. Anisovich, "The Lightest scalar glueball," Phys. Usp. 41 (1998) 419 [Usp. Fiz. Nauk 168 (1998) 481]

[186] P. Abbon et al. [COMPASS Collaboration], "The COMPASS experiment at CERN," Nucl. Instrum. Meth. A 577 (2007) 455

[187] A. B. Kaidalov and Y. .A. Simonov, "Glueball masses and Pomeron trajectory in nonperturbative QCD approach," Phys. Lett. B 477 (2000) 163 [hep-ph/9912434].

[188] A. Aktas et al. [H1 Collaboration], "Dijet Cross Sections and Parton Densities in Diffractive DIS at HERA," JHEP 0710 (2007) 042

[189] S. Chekanov et al. [ZEUS Collaboration], "A QCD analysis of ZEUS diffractive data," Nucl. Phys. B 831 (2010) 1

[190] A. D. Martin, M. G. Ryskin and G. Watt, "Diffractive parton distributions from H1 data," Phys. Lett. B 644 (2007) 131

[191] G. Hanson et al. [Mark-I Collaboration], "Evidence for Jet Structure in Hadron Production by e+ e- Phys. Rev. Lett. 35 (1975) 1609

[192] R. Brandelik et al. [TASSO Collaboration], "Evidence for Planar Events in e+ e- Annihilation at High-Energies," Phys. Lett. B 86 (1979) 243;

D. P. Barber et al. [MARK J Collaboration], "Discovery of Three Jet Events and a Test of Quantum Chromodynamics at PETRA Energies," Phys. Rev. Lett. 43 (1979) 830;

C. Berger et al. [PLUTO Collaboration], "Evidence for Gluon Bremsstrahlung in e+ eAnnihilations at High-Energies," Phys. Lett. B 86 (1979) 418;

W. Bartel et al. [JADE Collaboration], "Observation of Planar Three Jet Events in e+ eAnnihilation and Evidence for Gluon Bremsstrahlung," Phys. Lett. B 91 (1980) 142

[193] V. A. Khoze and W. Ochs, "Perturbative QCD approach to multiparticle production," Int. J. Mod. Phys. A 12 (1997) 2949

[194] J. Gallicchio and M. D. Schwartz, "Pure Samples of Quark and Gluon Jets at the LHC," JHEP 1110 (2011) 103

[195] W. Ochs and P. Minkowski, "Glueballs from Gluon Jets at the LHC," XIV Int. Conf. on Hadron Specrtroscopy (hadron 2011), 13-17 June 2011, Munich, Germany), arXiv:1108.0589 [hep-ph]

[196] P. Roy and T. F. Walsh, "Spotting Glueballs In Collinear Gluon Jets," Phys. Lett. B 78 (1978) 62 ;

C. Peterson and T. F. Walsh, "Model Of A Nonperturbative Gluon Jet," Phys. Lett. B 91 (1980) 455

[197] P. Roy and K. Sridhar, "A New proposal for glueball exploration in hard gluon fragmentation," JHEP 9907 (1999) 013;

H. Spiesberger and P. M. Zerwas, "Gluon fragmentation to gluonium," Phys. Lett. B 481 (2000) 236

[198] M. Acciarri et al. [L3 Collaboration], "Measurement of eta production in two and three jet events 
from hadronic Phys. Lett. B 371 (1996) 126;

R. Barate et al. [ALEPH Collaboration], "Inclusive production of pi0, eta, eta-prime (958), $\mathrm{K} 0(\mathrm{~S})$ and lambda in two jet and three jet events from hadronic Z decays," Eur. Phys. J. C 16 (2000) 613;

G. Abbiendi et al. [OPAL Collaboration], "Multiplicities of pi0, eta, K0 and of charged particles in quark and gluon jets," Eur. Phys. J. C 17 (2000) 373

[199] P. Minkowski and W. Ochs, "Gluon fragmentation into glueballs and hybrid mesons," Phys. Lett. B 485 (2000) 139

[200] J. Abdallah et al. [DELPHI Collaboration], "Study of Leading Hadrons in Gluon and Quark Fragmentation," Phys. Lett. B 643 (2006) 147;

B. Buschbeck and F. Mandl, in: Y. Bai, M. Yu, Y.Wu (Eds.), "Study of gluon fragmentation and color octet neutralization in DELPHI," Proceedings of the 31st Int. Symposium of Multiparticle Dynamics, Datong, China, Sept. 2001, World Scientific, Singapore, 2002, p.50; hep-ph/0111408

[201] G. Abbiendi et al. [OPAL Collaboration], "Tests of models of color reconnection and a search for glueballs using gluon jets with a rapidity gap," Eur. Phys. J. C 35 (2004) 293

[202] S. Schael et al. [ALEPH Collaboration], "Test of Colour Reconnection Models using Three-Jet Events in Hadronic Z Decays," Eur. Phys. J. C 48 (2006) 685

[203] T. Sjostrand, "High-energy physics event generation with PYTHIA 5.7 and JETSET 7.4," Comput. Phys. Commun. 82 (1994) 74

[204] D. Acosta et al. [CDF Collaboration], "Measurement of charged particle multiplicities in gluon and quark jets in $p \bar{p}$ collisions at $\sqrt{s}=1.8 \mathrm{TeV}$," Phys. Rev. Lett. 94 (2005) 171802

[205] A. Boehrer, "Inclusive particle production in hadronic decays of the Z boson at LEP-1," Phys. Rept. 291 (1997) 107

[206] S. J. Brodsky and G. R. Farrar, "Scaling Laws at Large Transverse Momentum," Phys. Rev. Lett. 31 (1973) 1153

[207] K. Ackerstaff et al. [OPAL Collaboration], "Production of $\mathrm{f}(0)(980), \mathrm{f}(2)(1270)$ and phi(1020) in hadronic Z0 decay," Eur. Phys. J. C 4 (1998) 19

[208] M. Ablikim et al. [BES Collaboration], "Evidence for f0(980) f0(980) production in chi(c0) decays," Phys. Rev. D 70 (2004) 092002

\section{List of reprinted figures}

The following figures are reprinted with kind permission of the copyright holders: American Physical Society:

"Readers may view, browse, and/or download material for temporary copying purposes only, provided these uses are for noncommercial personal purposes. Except as provided by law, this material may not be further reproduced, distributed, transmitted, modified, adapted, performed, displayed, published, or sold in whole or part, without prior written permission from the American Physical Society."

Fig. 1, from [18], C. J. Morningstar and M. J. Peardon, "The Glueball spectrum from an anisotropic lattice study," http://link.aps.org/abstract/PRD/v60/e034509;

Figs. 2,3, from [35], C. Amsler and F. E. Close, "Is $f_{0}(1500)$ a scalar glueball?," http://link.aps.org/abstract/PRD/v53/p295;

Fig. 5c, from [103], P. del Amo Sanchez et al., "Analysis of the $D^{+} \rightarrow K^{-} \pi^{+} e^{+} \nu_{e}$ decay channel," http://link.aps.org/abstract/PRD/v83/e072001;

Fig. 16, from [83], R. Aaij et al., "Analysis of the resonant components in $B_{s} \rightarrow J / \psi \pi^{+} \pi^{-}$," DOI: 10.1103/PhysRevD.86.052006.

Elsevier B. V.:

Fig. 6, from [5], E. Klempt and A. Zaitsev, "Glueballs, Hybrids, Multiquarks. Experimental facts versus QCD inspired concepts;"

Fig. 7c, from [129], T. A. Armstrong et al., "Evidence for $\eta \eta$ resonances in $\bar{p} p$ annihilations at 
$2950<\sqrt{s}<3620 \mathrm{MeV} ;$

Figs. 10, 11, from [102], W. Ochs, "Scalar mesons: In search of the lightest glueball;"

Fig. 18, from [172], D. Barberis et al., "A Partial wave analysis of the centrally produced $\pi^{0} \pi^{0}$ system in p p interactions at $450-\mathrm{GeV} / \mathrm{c}$;"

Fig. 22, from [199], P. Minkowski and W. Ochs, "Gluon fragmentation into glueballs and hybrid mesons,"

Figs. 23, 25a, from [200], J. Abdallah et al., "Study of Leading Hadrons in Gluon and Quark Fragmentation;"

Springer Science + Business Media B.V.:

Figs. 7a,b, from [128], C. Amsler et al., "Proton anti-proton annihilation at 900-MeV/c into $\pi^{0} \pi^{0} \pi^{0}, \pi^{0} \pi^{0} \eta$ and $\pi^{0} \eta \eta ;$

Fig. 9, from [150], D. Alde et al., "Study of the $\pi^{0} \pi^{0}$ system with the GAMS-4000 spectrometer at 100-GeV;"

Fig. 12, from [154], P. Buettiker et al., "A new analysis of $\pi K$ scattering from Roy and Steiner type equations;"

Fig. 14, from [48], P. Minkowski and W. Ochs, "B decays into light scalar particles and glueball";

Fig. 24, from [202], S. Schael et al., "Test of Colour Reconnection Models using Three-Jet Events in Hadronic Z Decays;"

Fig. 25b, from [201], G. Abbiendi et al., "Tests of models of color reconnection and a search for glueballs using gluon jets with a rapidity gap." 